\title{
DETECTABILITY PREDICTION FOR A THERMOACOUSTIC SENSOR IN THE BREAZEALE NUCLEAR REACTOR POOL
}

James Smith

Joshua Hrisko

Steven Garrett

Prepared for

U.S. Department of Energy Office of Nuclear Energy

March 2016 


\section{Disclaimer}

This information was prepared as an account of work sponsored by an agency of the U.S. Government. Neither the U.S. Government nor any agency thereof, nor any of their employees, makes any warranty, expressed or implied, or assumes any legal liability or responsibility for the accuracy, completeness, or usefulness, of any information, apparatus, product, or process disclosed, or represents that its use would not infringe privately owned rights. References herein to any specific commercial product, process, or service by trade name, trade mark, manufacturer, or otherwise, does not necessarily constitute or imply its endorsement, recommendation, or favoring by the U.S. Government or any agency thereof. The views and opinions of authors expressed herein do not necessarily state or reflect those of the U.S. Government or any agency thereof. 
INL/EXT-16-37905

Revision: 0

\begin{abstract}
Laboratory experiments have suggested that thermoacoustic engines can be incorporated within nuclear fuel rods. Such engines would radiate sounds that could be used to measure and acoustically-telemeter information about the operation of the nuclear reactor (e.g., coolant temperature or fluxes of neutrons or other energetic particles) or the physical condition of the nuclear fuel itself (e.g., changes in temperature, evolved gases) that are encoded as the frequency and/or amplitude of the radiated sound [IEEE Measurement and Instrumentation 16(3), 18-25 (2013)]. For such acoustic information to be detectable, it is important to characterize the vibroacoustical environments within reactors. Measurements will be presented of the background noise spectra (with and without coolant pumps) and reverberation times within the 70,000 gallon pool that cools and shields the fuel in the 1 MW research reactor on Penn State's campus using two hydrophones, a piezoelectric projector, and an accelerometer. Several signal-processing techniques will be demonstrated to enhance the measured results. Background vibrational measurement were also taken at the $250 \mathrm{MW}$ Advanced Test Reactor, located at the Idaho National Laboratory, using accelerometers mounted outside the reactor's pressure vessel and on plumbing will also be presented. The detectability predictions made in the thesis were validated in September 2015 using a nuclear fission-heated thermoacoustic sensor that was placed in the core of the Breazeale Nuclear Reactor on Penn State's campus. Some features of the thermoacoustic device used in that experiment will also be revealed. [Work supported by the U.S. Department of Energy.]
\end{abstract}


The Pennsylvania State University

The Graduate School

College of Engineering

\title{
DETECTABILITY PREDICTION FOR A THERMOACOUSTIC SENSOR IN THE BREAZEALE NUCLEAR REACTOR POOL
}

\author{
A Thesis in \\ Acoustics \\ by \\ Joshua Eric Hrisko \\ (c) 2015 Joshua Eric Hrisko \\ Submitted in Partial Fulfillment \\ of the Requirements \\ for the Degree of \\ Master of Science
}

May 2016 
The thesis of Joshua Eric Hrisko was reviewed and approved* by the following:

\author{
Steven L. Garrett \\ Professor of Acoustics \\ Thesis Advisor \\ Michele Vigeant \\ Assistant Professor of Acoustics \\ Assistant Professor of Architectural Engineering
}

Karl Reichard

Assistant Professor of Acoustics

Robert Smith

Research Associate

Special Signatory

Victor W. Sparrow

Professor of Acoustics

Director of the Graduate Program in Acoustics

*Signatures are on file in the Graduate School. 


\section{Abstract}

This thesis reports the first quantitative measurements of the vibroacoustic background noise levels and the reverberation time in the 70,000 gallon $\left(265 \mathrm{~m}^{3}\right)$ pool used to cool the Breazeale Nuclear Reactor on Penn State's University Park campus. These measurements are used to provide an estimate for the detectability of a pure tone generated by a thermoacoustic engine that will be placed in the E-6 fuel position within that reactor's core to act as a self-powered, acoustically-telemetered thermoacoustic sensor (TAC Sensor) capable of measuring coolant temperature (based on the radiated frequency) and neutron flux (based on the radiated amplitude).

Background noise levels within the pool were measured with two hydrophones placed at the bottom of the pool. Vibration levels were measured with a single-axis accelerometer mounted on a partially-submerged instrumentation tower. An underwater sound source was also placed at the bottom of the pool and was used to radiate pure tones from $1.4 \mathrm{kHz}$ to $2.4 \mathrm{kHz}$ and broadband (white) noise. The decay of those tones allowed estimation of the reverberation time, which is approximately $140 \pm 50 \mathrm{~ms}$ in the frequency range between 1.2 $\mathrm{kHz}$ and $2.4 \mathrm{kHz}$. The first 100 lowest frequency standing wave normal modes of the pool were calculated. The measured reverberation times were used to estimate the Schroeder frequency $\left(f_{c}=230 \mathrm{~Hz}\right)$ that was well below the design frequency of the TAC Sensor. Therefore, the sound field created by a TAC Sensor should be dominantly diffuse, rather than modal, at distances greater than the critical distance from the reactor core $\left(r_{c} \approx 1.6\right.$ $\mathrm{m}=5.25 \mathrm{ft})$.

In addition to real-time display of all three vibroacoustic sensor outputs on an oscilloscope and a 4-channel dynamic signal analyzer, all signals were recorded digitally at CD-quality (16-bit, 44.1 kilosamples/second) for subsequent analysis. Custom analyses using power spectral density techniques, spectrograms, and waterfall plots are described. 
High-pass analog filtering of sensor signals prior to recording is recommended to reduce background noise below $1 \mathrm{kHz}$, thus increasing the available dynamic range of the digital recorder. Narrow-band filtration of the digitized signal is recommended for tracking the TAC Sensor signal's frequency and amplitude, especially if multiple TAC Sensors are frequency-division-multiplexed in the future to simultaneously monitor several positions throughout a reactor's core.

This thesis concludes that a TAC Sensor's signal should be detectable in the Breazeale Reactor based on the design detailed in the Idaho National Laboratory Technical Report No. INL-LTD-15-34228 (March 2015). The technical report outlines many of the necessary conclusions drawn from the calculations and measurements made in this thesis, and serves as the impetus for fabrication of the TAC sensor and how it will behave in the Breazeale acoustic environment. 


\section{Table of Contents}

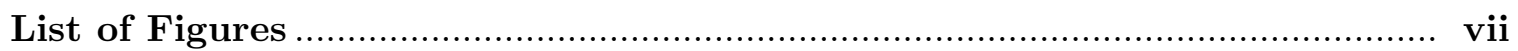

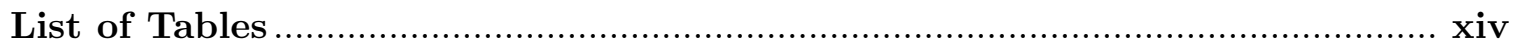

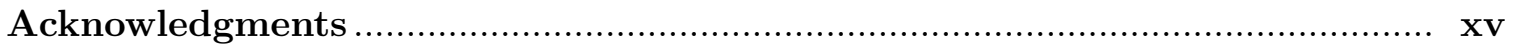

\section{Chapter 1}

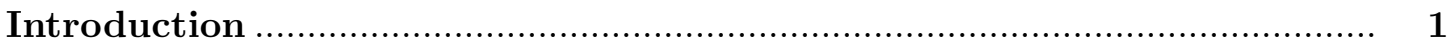

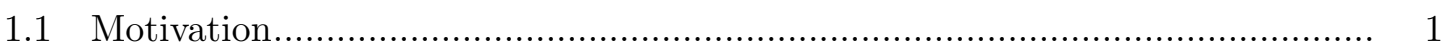

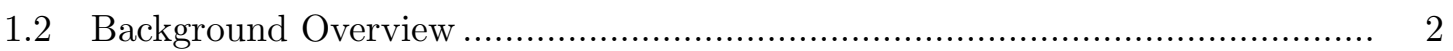

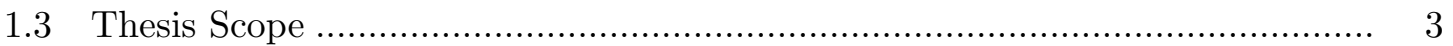

\section{Chapter 2}

Reactor Pool Sound Field Analysis

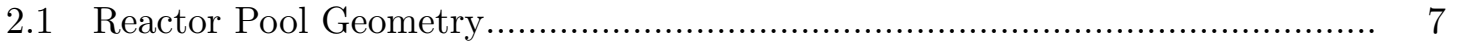

2.1.1 Architectural Overview......................................................... 7

2.1.2 Geometric Assumptions................................................................. 8

2.2 Identifying a Suitable Frequency Band ................................................. 9

2.2.1 Rigid and Pressure-Release Boundaries....................................... 9

2.2.2 Modal Characteristics of the Pool ................................................ 12

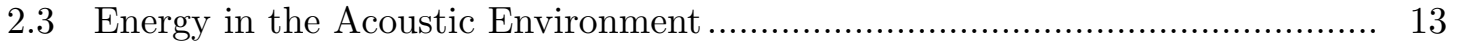

2.3.1 Conserving Energy through Absorption ....................................... 14

2.3.2 Steady-State Condition ........................................................... 18

2.4 Thermoacoustic Fuel-Rod Resonator ..................................................... 21

\section{Chapter 3}

Breazeale Reactor Vibroacoustic Measurements ................................. 23

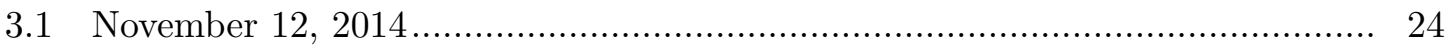

3.1.1 Coolant Pump Deactivated .................................................... 27

3.1.2 Coolant Pump Activated .......................................................... 32

3.1.3 Frequency Content of Pump Noise ................................................... 37

3.1.4 Reverberation Measurements ............................................................. 40

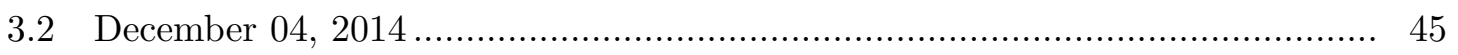

3.2.1 Reverberation Measurements After Relocation of Hydrophone-008 ..... 45

3.2.2 Frequency Sweep Measurement ...................................................... 49

3.3 Reverberation Analysis and Frequency Filtration...................................... 49

3.3.1 Reverberation Time and Direct Sound Decay …............................... 50

3.3.2 Filter Techniques ................................................................ 51

3.3.3 Spectrogram vs. Power Spectrum .............................................. 55 


\section{Chapter 4}

Feasibility of Thermoacoustic Sensor Signal Detection ….................... 59

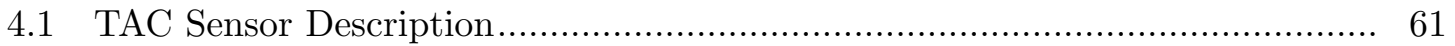

4.2 TAC Sensor Output Power and Detectability ................................................. 63

\section{Chapter 5}

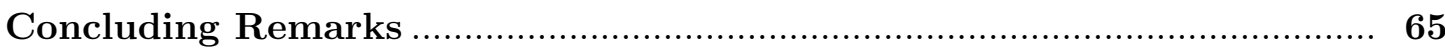

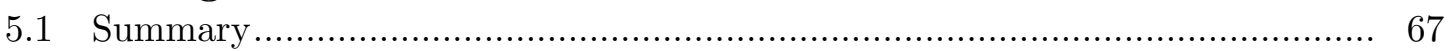

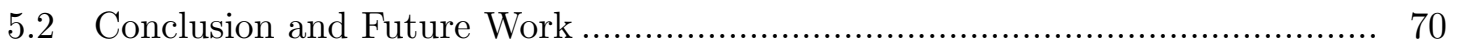

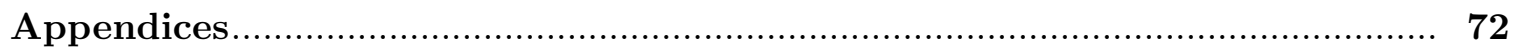

Appendix A

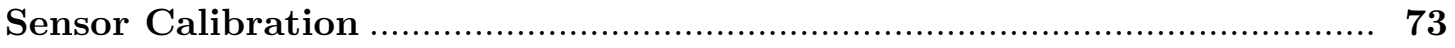

Appendix B

Instrumentation used for Reactor Vibroacoustic Measurements .............. 74

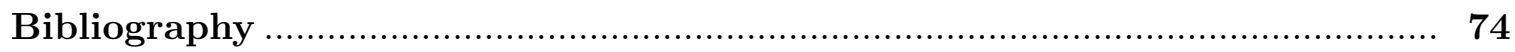




\section{List of Figures}

1.1 Fukushima Daiichi Nuclear Power Plant diagram showing the location of the reactors and the layout of the natural disaster prevention system. The anti-earthquake system, the emergency response center, and the breakwater partitions were constructed to prevent nuclear disasters of the type experi-

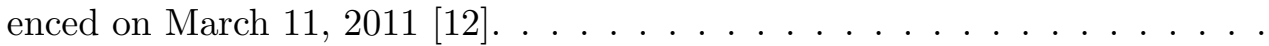

1.2 Photo of the resonator used for Ali and Garrett's experiments [2]. The heating element is the $\mathrm{NiCr}$ wire glowing in the foreground, and the stack is the larger honeycomb-like material to the right of the heater wire. This device is a simplified version of the thermoacoustic fuel rod resonator that will be placed in the Breazeale reactor, but served as the first step in showing the relation between the temperature and frequency of a resonator submerged in water. . . . . . . . . . . . . . . . . .

2.1 Simple geometric representation of the Breazeale Nuclear Reactor pool. The depth of the pool is $24 \mathrm{ft}(7.32 \mathrm{~m})$ and its sides are made of steel-reinforced concrete $1.5 \mathrm{ft}(0.46 \mathrm{~m})$ thick. The southernmost wall located in the south bay, labeled -4-, is $3.5 \mathrm{ft}(1.07 \mathrm{~m})$ thick to shield the neutron beam ports. Typically, the core is located near the center of the south bay, labeled -6-. .

2.2 Simple geometric representation showing the adiabatic transformation from hexagonal to rectangular floor plan. . . . . . . . . . . . .

2.3 Illustration showing the effects of absorption on a system. The power output is a constant sound source emitting 'sound power droplets' into a bucket that represents the acoustic environment. An average sound level is produced, based on an average absorption. The power absorbed and the average measured sound power add to equal the total acoustic power input [21]. . .

2.4 First 100 calculated modes of the BRP. The different colors distinguish between adjacent modes and the modal amplitudes are arbitrary. Notice that the modes begin to crowd as early as $180 \mathrm{~Hz}$, and are densely overcrowded by the $500 \mathrm{~Hz}$ mark. For the reactor pool, it is evident that the Schroeder frequency should be lower than the thermoacoustic sensor's resonance frequency. . . . . . . . . . . . . . . . . . 
2.5 Illustration of a half-wavelength resonator that shows a zoomed-in view of the compression and expansion of a gas within the resonator's stack material. Four processes take place within the stack: 1. movement of gas toward the hot end of the stack, accompanied by compression of gas; 2. transfer of heat from the stack to the gas; 3. movement of gas toward the cold end of stack, accompanied by expansion of gas; 4. transfer of heat from the gas to the stack. The stack is designed so that the pores have gaps only a few thermal diffusion distances, $\delta_{\kappa}$, to permit ample thermal interaction. The heat exchangers promote oscillation by removing and adding heat to the ends of the stack $[2] \ldots \ldots \ldots \ldots \ldots$

3.1 Transducer placement in the Breazeale reactor pool during the November 12, 2014 measurements. The red ovals represent the respective transducer. All except the accelerometer were placed at the bottom of the pool. The accelerometer was placed on an instrumentation dolly that was in direct contact with the water (see Fig. 3.9). A 3-dimensional rendering of the source and sensor locations is provided in Fig. 3.2. . . . . . . . . . .

3.2 Transducer placement in the Breazeale reactor pool during the November 12, 2014 and December 04, 2014 measurements. The left-hand side of the figure represents the north bay and the right-hand side the south bay. The red objects represent the respective transducer. H-008(1) represents the hydrophone placement on November $12^{\text {th }}$, and H-008(2) represents the hydrophone after it was move for the December $4^{\text {th }}$ measurements. For more information on the reactor pool visualization refer to the Fig. 3.10 and Appendix B. . . . . . . . . . . . . . . . . .

3.3 Time series of tri-tone frequencies while the coolant pump was deactivated. From top to bottom: hydrophone-008, hydrophone-007, and the accelerometer. The long time series was recorded to ensure ample signal-to-noise ratio.

3.4 Power spectra from the time records of Fig. 3.3 for all three transducers while the tri-tone was emitted during the coolant pump deactivation. Each tonal frequency was more than two orders of magnitude above the noise for all three transducers. . . . . . . . . . . . . . . . . .

3.5 Power spectrum of a 23 second time window measurement of hydrophone-008 during the pump off measurement based on the time history of Fig. 3.3. . .

3.6 Power spectrum of a 23 second time window measurement of hydrophone-007 during the pump deactivation measurement based on the time history of Fig.

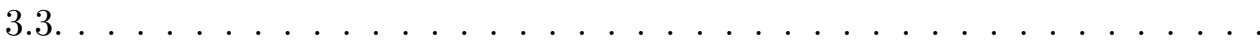

3.7 Power spectrum of a 23 second time window measurement of the accelerometer during the pump deactivation measurement based on the time history of Fig. 3.3. . . . . . . . . . . . . . . . . . . .

3.8 Spectrogram of a 23 second time window measurement of hydrophone-008 with the coolant pump deactivated. A 0.37 second Hann window $(2.7 \mathrm{~Hz}$ frequency bin) and $85 \%$ overlap between time records was used to enhance smoothing. No averaging between power spectra was done. . . . . . . . . .

3.9 Accelerometer placement on the instrumentation dolly in the Breazeale reactor pool. . . . . . . . . . . . . . . . . . 
3.10 AutoCAD ${ }^{\circledR}$ rendering of the primary noise sources due to pump fluid flow. The left side of the figure represents the south bay where the reactor core is typically located. The ${ }^{16} \mathrm{~N}$ pump discharges toward the top of the core assembly, while the filtration pump discharges from the bottom of the pool. The coolant pump discharges from the north bay where the transducers were located. . . . . . . . . . . . . . . . . .

3.11 Time series of tri-tone frequencies after coolant pump activation. The axes are identical to those in Fig. 3.3, from top to bottom: hydrophone-008, hydrophone-007, and the accelerometer. Notice the fluctuations in H-007. This was due to interaction between the hydrophone's cable and a nearby randomly-bobbing pipe. Some interference caused the time series to fluctuate greatly, but the frequency domain is minimally impacted by the slow amplitude modulation, as shown Fig. 3.13. . . . . . . . . . . . .

3.12 Power spectrum of each transducer after the pump activation. No debilitating effects are present when the coolant pump is running. Each transducer still shows a high signal-to-noise ratio. . . . . . . . . . . . .

3.13 Spectrogram of a 200 second time window measurement of hydrophone-007 after the pump activation. A 0.186 second long $(5.38 \mathrm{~Hz}$ bin) Hann window was implemented without averaging and $50 \%$ overlap between time records was used to enhance smoothing. . . . . . . . . . . . . . . .

3.14 Power spectrum of a 226 second time window measurement of hydrophone008 while the coolant pump was operating. . . . . . . . . . . . .

3.15 Power spectrum of a 226 second time window measurement of hydrophone007 while the coolant pump was activated. . . . . . . . . . .

3.16 Power spectrum of a 226 second time window measurement of the accelerometer while the coolant pump was activated. . . . . . . . . . . .

3.17 Power spectra for H-008 during pump-on and pump-off measurements. The noise floor is raised when the coolant pump is activated, but the tri-tone frequencies are seemingly unaffected and remain resolvable. . . . . . . . . .

3.18 Power spectra for H-007 during pump-on and pump-off measurements. The noise floor is raised when the coolant pump is activated, but the tri-tone frequencies are unaffected and remain resolvable. . . . . . . . . . . .

3.19 Power spectra for the accelerometer during pump-on and pump-off measurements. The noise floor is raised when the coolant pump is activated, but the tri-tone frequencies are well above the noise floor. It appears that a high frequency contribution to the noise exists between $2.5 \mathrm{kHz}$ and $3.5 \mathrm{kHz}$. This may be attributed to vibrational modes of the instrumentation dolly and to the nonlinear increase in accelerometer response with increasing frequency. .

3.20 Low frequency power spectra for H-008 during pump-on and pump-off measurements. The broadband noise floor absorbs most tonals in the low frequency range. This low frequency noise can be high-pass filtered to prevent saturation of the signal-conditioning and data acquisition electronics for future measurements. . . . . . . . . . . . . . . . . .

3.21 Low frequency power spectra for H-007 during pump-on and pump-off measurements. The broadband noise floor absorbs most tonals in the low frequency range. This low frequency noise can be high-pass filtered to prevent saturation of the signal-conditioning and data acquisition electronics for future measurements. . . . . . . . . . . . . . . . . 
3.22 Low frequency power spectra for the accelerometer during pump-on and pump-off measurements. The broadband noise floor absorbs most tonals in the low frequency range. This low frequency noise can be high-pass filtered to prevent saturation of the signal-conditioning and data acquisition electronics for future measurements. . . . . . . . . . . . . . . . .

3.23 Time series of reverberation measurements without the coolant pump running. Each group of waveforms represents a separate steady-state excitation and its corresponding cutoff. The first five periods were frequencies ranging from $1.6 \mathrm{kHz}$ to $2.4 \mathrm{kHz}$ in steps of $200 \mathrm{~Hz}$, and the sixth period was excited by broadband white noise. . . . . . . . . . . . . . .

3.24 Power spectra showing the five frequency peaks from $1.6 \mathrm{kHz}-2.4 \mathrm{kHz}$ in steps of $200 \mathrm{~Hz}$ for all three transducers. . . . . . . . . . . . . . .

3.25 Time series spectrogram of H-008 for the reverberation measurements with a 0.37 second (2.7 Hz bin) Hann window (no averaging) and $80 \%$ overlap between time records. The change in bin size varied based on visual inspection for smoothing in the spectrogram. Each slice in time represent a separate steady-state excitation and the corresponding cutoff. The first five periods between $0 \mathrm{~s}$ and $130 \mathrm{~s}$ correspond to excitation at frequencies ranging from $1.6 \mathrm{kHz}-2.4 \mathrm{kHz}$ in steps of $200 \mathrm{~Hz}$. The sixth period from $180 \mathrm{~s}$ to $230 \mathrm{~s}$ corresponds to broadband white noise. . . . . . . . . . . .

3.26 An example of the time series cutoff of $\mathrm{H}-007$ during the $2 \mathrm{kHz}$ reverberation measurement. The red line indicates the time when the sound source was cutoff, and everything after is considered the decay and reverberation of the reactor pool. . . . . . . . . . . . . . . . .

3.27 Transducer placement in the Breazeale reactor pool during the December 04, 2014 measurements. The red ovals represent the labeled transducer. H008 has been relocated to decouple it from the direct path to the sound source. Note: the accelerometer was not required for these reverberation time measurements. . . . . . . . . . . . . . . . . . .

3.28 Time series for each reverberation segment after H-008 was relocated. Each time interval represents a separate steady-state drive and its corresponding cutoff. The five periods contained frequencies ranging from $1.6 \mathrm{kHz}-2.4 \mathrm{kHz}$ in steps of $200 \mathrm{~Hz} . \ldots \ldots \ldots \ldots \ldots$

3.29 Power spectra showing the five frequency peaks from $1.6 \mathrm{kHz}-2.4 \mathrm{kHz}$, in steps of $200 \mathrm{~Hz}$, for all three transducers. . . . . . . . . . . . .

3.30 Time series spectrogram of $\mathrm{H}-008$ for the reverberation measurements after relocation. A 0.37 second Hann window $(2.7 \mathrm{~Hz}$ frequency bin) with $80 \%$ overlap between time records was used. No averaging between power spectra was done. Each slice in time represents a separate steady-state setup and its corresponding cutoff. The five records were driven at frequencies ranging from $1.6 \mathrm{kHz}-2.4 \mathrm{kHz}$ in steps of $200 \mathrm{~Hz}$. . . . . . . . . . . .

3.31 Example of the time series cutoff of $\mathrm{H}-008$ during the $2 \mathrm{kHz}$ reverberation measurement after the hydrophone was relocated. The red line indicates the time when the sound source was cut off, and everything after is the decay characterizing the reverberation in the reactor pool. . . . . . . . . 
3.32 Time series spectrogram of H-008 for the swept sine measurement after relocation. A 0.186 second long $(5.38 \mathrm{~Hz}$ bin) Hann window (no averaging) and $95 \%$ overlap was used. $95 \%$ overlap with a 0.186 second long window was used to capture enough cycles of the frequencies of interest, but smooth them enough to be able to observe subtle changes with high time resolution, which will be important for quick changing processes such as a frequency sweep. Subsequently, the sound source was swept from $1 \mathrm{kHz}$ to $3 \mathrm{kHz}$ to simulate a rapid rise in coolant temperature. The clarity of the sweep is a good indicator of the ability to monitor temperature in a nuclear reactor. Even in the low frequency end amidst the pump noise $(\mathrm{f}<1.5 \mathrm{kHz})$, a clear signal evident at the chosen drive level. . . . . . . . . . . . .

3.33 Example of the decibel level vs. time cutoff method applied to the signal from $\mathrm{H}-008$ during the $2 \mathrm{kHz}$ reverberation measurement after the hydrophone was relocated. The line approximates the slope of the energy decay. The slopes were approximated by using a slope-intercept method at two different points on the decay curve. . . . . . . . . . . . . . . . .

3.34 A longer view of the time series cutoff for $\mathrm{H}-008$ during the $2 \mathrm{kHz}$ reverberation measurement (after hydrophone-008 was relocated). The line indicates the slope of the amplitude decay after the cutoff that characterizes the losses in the pool. . . . . . . . . . . . . . . . . .

3.35 Time series spectrogram of $\mathrm{H}-008$ during the activation of the ${ }^{16} \mathrm{~N}$ diffuser pump. A 0.37 second $(2.7 \mathrm{~Hz}$ bin) Hann window (no averaging) and $90 \%$ overlap of time records was used. The sound source was generating a 1.6 $\mathrm{kHz}$ sine wave when the pump was activated. The activation of the pump is seen right before the 4 second mark. Notice the low frequency content of the pump signal. The frequency content after the 4 second mark is representative of the largest amount of noise during normal reactor operation. . . . . . . .

3.36 Low-frequency filtered time series spectrogram of H-008 during the activation of the ${ }^{16} \mathrm{~N}$ diffuser pump. A 0.37 second $(2.7 \mathrm{~Hz}$ bin) Hann window (no averaging) and $90 \%$ overlap of time records was used, as well as a 3rd order butterworth bandpass filter that rolls off at $1.2 \mathrm{kHz}$ and $20 \mathrm{kHz}$. The sound source was generating a $1.6 \mathrm{kHz}$ sine wave while the pump was activated. When compared to Fig. 3.35, the effect of the filter makes the background noise nearly independent of frequency. . . . . . . . . . . . . . .

3.37 Comparison of different filters for the power spectra of the five frequency peaks $(1.6 \mathrm{kHz}-2.4 \mathrm{kHz})$ during reverberation measurements of $\mathrm{H}-008$. The first plot at the top is the unfiltered power spectrum. The plot in the middle is a simple single-pole high-pass filter with a $-3 \mathrm{~dB}$ cutoff frequency at 1.2 $\mathrm{kHz}$, and the last plot is a butterworth bandpass filter that has cutoffs at 1.2 $\mathrm{kHz}$ and $20 \mathrm{kHz} . \ldots \ldots \ldots \ldots \ldots$

$3.381 .6 \mathrm{kHz}$ signal after narrow-band filtering. The amplitude has been attenuated (see Table 3.3). This method of 1/15-octave filtering can be used to track relative amplitudes of a specific frequency as it changes with time. . .

3.39 Power spectrum showing the indistinct frequency sweep. There is no clear differentiation between the noise and the energy of the signals because the frequency of the tonal is increasing continuously during acquisition of the time record. . . . . . . . . . . . . . . . . . . . 
3.40 Spectrogram of a frequency sweep from $1 \mathrm{kHz}-3 \mathrm{kHz}$. The spectrogram functions as a better method for illustrating frequency changes, whereas the power spectrum is not useful if there are significant frequency changes during acquisition of the time record. . . . . . . . . . . . . . . . . .

3.41 Top: power spectrum during the ${ }^{16} \mathrm{~N}$ pump activation. Notice that the lack of time discrimination makes it nearly impossible to see that the pump was activated within the measurement period. Bottom: spectrogram showing the frequency behavior over time. It is easy to see that there was a broadband disturbance that started around the 3.5 second mark. Both power spectrum and spectrogram are important for the tracking and monitoring of acoustic signals. . . . . . . . . . . . . . . . . . .

3.42 Waterfall plot showing the ${ }^{16} \mathrm{~N}$ diffuser pump activation. The waterfall method shows frequency, time, and amplitude values. This type of plot could be useful to a reactor operator monitoring the reactor in real time looking for simultaneous frequency-amplitude changes over time. . . . . . . . . . .

3.43 Sound propagation path in the Breazeale reactor pool. Path A shows the direct path from the underwater sound source to hydrophone-008 (after relocation), and Path B shows the direct path from the sound source to hydrophone007. The propagation distances are approximated for each path in Eqs. 3.2 and $3.3 \ldots \ldots \ldots \ldots \ldots \ldots \ldots \ldots$

3.44 Time series showing the overlay of hydrophone-007 and -008 during the initiation of a reverberation measurement. The time delay between the hy-

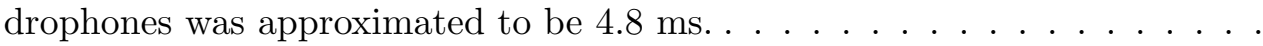

3.45 Time series showing the overlay of hydrophone-007 and -008 during the cutoff of a reverberation measurement. The time delay between the hydrophones was again approximated to be $4.8 \mathrm{~ms}$. Notice the complex nature of the waveform after cutoff. . . . . . . . . . . . . . . . .

4.1 Full model of the thermoacoustic resonator to be tested in the Breazeale reactor pool. The ends of the resonator pictured show the leaf spring suspension system that connects the inside resonator to the slotted fuel pin. The fuel pin is slotted to permit sound propagation into the surrounding water. The stack and nuclear fuel enclosure are pictured on the right end of the resonator. 62

4.2 A zoomed-in view of the resonator shown in Fig. 4.1. The inner resonator is very similar to that of the Ali apparatus, apart from the heating element. The slotted outer shell is shown in contact with the leaf spring suspension system. This allows the resonator to oscillate without interference from the slotted outer shell. . . . . . . . . . . . . . . . . . .

4.3 Leaf spring and fuel enclosure drawings for the fuel rod resonator. The leaf spring acts as the suspension system that allows the resonator to freely oscillate inside the slotted fuel pin. The fuel enclosure acts as both a casing for the nuclear pellets and a heat-exchanger on the flowing gas in the resonator. The leaf spring is the connecting component between the resonator and the fuel pin. . . . . . . . . . . . . . . . . . . 
4.4 Acoustic standing wave predicted by DELTAEC within the nuclear-powered thermoacoustic sound source with an overall resonator length of $21 \mathrm{~cm}$ (10 in). The green line is the gas-filled cross-sectional area $\left(\times 10^{4} \mathrm{~m}^{2}\right)$, pink is absolute temperature $\left(\times 10^{-2} \mathrm{~K}\right)$, black is pressure amplitude $\left(\times 10^{-4} \mathrm{~Pa}\right)$, purple is acoustic power (watts), and dashed blue is acoustic volume velocity $\left(\times 10^{3} \mathrm{~m}^{3} / \mathrm{s}\right)$, all as a function of position within the resonator. The model predicts that the gas exerts a peak force of $57 \mathrm{~N}$ on the resonator at a frequency of $1,566 \mathrm{~Hz} . \ldots \ldots \ldots \ldots$

4.5 Fuel pin locations in the core of the Breazeale Nuclear Reactor. The TAC Sensor will be placed in the E-6 location. . . . . . . . . . . . . . . .

5.1 Plot made by R. Ali for a thermoacoustic resonator in a calorimeter. Temperature and frequency are shown in red and blue, respectively. The resonator was electrically heated and showed linearity in both frequency and gas temperature. The ability to track these changes suggested an auspicious future and motivated this current thesis. . . . . . . . . . . . . . .

B.1 Data flow during the Breazeale Reactor Pool measurements. The oscilloscope, digital signal analyzer, and digital recorder were hardware that were cross referenced for real-time monitoring and post-processing analysis. . . .

B.2 Classic drawing of the Breazeale Reactor [24]. The figure shows all of the essential functions of an open-pooled research reactor. It was also a model for the drawings made in Figs. B.3-B.6. . . . . . . . . . . . . . . . .

B.3 AutoCAD ${ }^{\circledR}$ drawing of the Breazeale Reactor Pool with the floor, walls, and main infrastructure. . . . . . . . . . . . . . .

B.4 AutoCAD ${ }^{\circledR}$ drawing of the Breazeale Reactor Pool with the sides taken down. This view allows one to view the pumps, transducers, and the core. .

B.5 Labeled transducers with the reactor core and pumps in view. This is identical to Fig. 3.2 in Sec. 3.2.1. . . . . . . . . . . . . . . . . . .

B.6 Labeled pumps and their location in the reactor pool. The N-16 diffuser pump was the most significant noise source of the three pumps (see Sec. 3.2). 77 


\section{List of Tables}

2.1 First nine modal frequencies of the Breazeale reactor pool. Three types of modes are demonstrated: axial (only one non-zero index), tangential (only one zero index), and oblique (no zero indices). Mode types are important for accurate room acoustic calculations in spaces inhabited by humans to establish perception and hearing, however, the current analysis does not necessitate distinction between them. . . . . . . . . . . . . . . .

3.1 Reverberation times for hydrophone-007. The low reverberation times suggested that the hydrophone was experiencing direct sound decay from the sound source, rather than representative behavior of the diffuse sound field in the reactor pool. In Sec. 3.2.1, hydrophone-008 is relocated and longer $T_{60}$ values are calculated. . . . . . . . . . . . . .

3.2 Reverberation times and the corresponding critical distances for hydrophone008 after relocation. The longer reverberation times suggest that this hydrophone experienced effects not fully understood in its previous location, such as low side lobes due to a directional sound source or back-scattering attenuation. Each critical distance is based on the measured reverberation time in the previous column. The average critical distance was $1.26 \mathrm{~m}$. All of the sensors were farther than $1.26 \mathrm{~m}$ from any noise or sound source, therefore concluding that the measurements were made in a diffuse sound field, and it is possible that the original assumption - that low reverberation times were a result of direct sound decay from the sound source - was incorrect. .

3.3 Amplitude comparison before and after 1/15-octave filtering. The amplitude attenuation can be reduced by increasing the filter bandwidth, but only a simple peak-detection algorithm and narrow filter are used here. . . . . . .

A.1 Calibration and instrumentation for the November 12, 2014 and December 4, 2014 experiments. The top four transducers were used in the experiments, and the bottom three were used as calibration devices before and after the

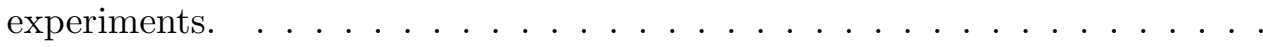




\section{Acknowledgements}

I would like to thank Dr. Steven Garrett for being the most honest and supportive advisor and mentor. He is the reason and result of my success as an engineer and a researcher. His patience and willingness to share his time and advice helped me to bond my academic and professional minds to flourish into a desired graduate of the Acoustics Program at Penn State. I would also like to thank Dr. James A. Smith and Idaho National Laboratory for their financial support and valuable input. Additionally, I thank Michael D. Heibel at Westinghouse Electric Company for the continuation of financial support and research, Mark Trump at Radiation Science and Engineering at Penn State for the time spent at the Breazeale reactor.

I would finally like to thank my parents for their unwavering support and guidance, even at the most fruitless times. I owe all of my success to the inherited tenacity from my father, and the inexorable aspirations acquired from my mother. I would also like to thank my brothers for being encouraging and relatable. Finally, without support from my friends and colleagues, this journey would have been much less enjoyable and not nearly as much fun. I thank everyone who made my graduate school experience both enjoyable and motivational, because at the end of the day if I don't love the people that surround me, it's hard to enjoy my work. I enjoyed every moment of the last two years, and I thank the my extensive support group for that. 


\section{Chapter 1 \\ Introduction}

\subsection{Motivation}

On March 11, 2011, the Fukushima Prefecture of Japan experienced one of humankind's

largest recorded disasters. The Great East Japan Earthquake marauded along the Japanese coast with a magnitude larger than anything the country had previously recorded. The Fukushima Daiichi Nuclear Power Plant, located on the country's eastern shore (see Fig. 1.1), was operating three of its six nuclear reactors when the earthquake struck. Reactors 1-3 were in operation during the seismic activity, and all three were properly shut down in accordance with typical emergency shutoff procedures. Backup generators and reactor cooling began; however, within 45 minutes of the earthquake a 15 meter tsunami hit the city coast and disabled nearly all electrical power. Reactors 1, 2, and 4 lost all electrical power immediately following the tsunami, and reactor 3 had a complete station blackout where only DC power remained. Within one day, reactor station 1 suffered a hydrogen explosion due to the inability to monitor and properly vent gas buildup in the reactor's core. Stations 3, and 4 also experienced these hydrogen explosions, and each subsequently released harmful radiation into the atmosphere and surrounding environment. The Fukushima Daiichi Plant was deemed a "major accident," the most severe rating on the International Nuclear and Radiological Event Scale [10][12].

A myriad of scientific papers were published on the disaster in Japan, and one in particular guided the research discussed in this thesis. As far back as March 2011, research on the feasibility of a nuclear powered acoustic resonator was conducted at Idaho National Laboratory and The Pennsylvania State University [22]. This resonator would be manufactured to resemble a fuel pin that could be placed in a nuclear reactor's core. The "fuel-rod resonator" would be thermally powered by nuclear fuel (or other fissionable material or gamma absorber) and have no electrical components or moving parts. This unique characteristic of the acoustic device was a response to the Fukushima accident, which was exacerbated by the inability to monitor the reactors after the tsumani caused power outages across the entire complex. The resonator would be part of the fuel assembly just as any other fuel pin, except that it would resonate and emit a single audible tone. This unique tone would allow reactor operators to monitor the behavior of the reactor without 
electrical power, and ultimately determine whether a disaster is imminent.

As this thesis will demonstrate, the Breazeale Nuclear Reactor provided the perfect venue to test the first nuclear powered acoustic resonator. To ensure that the resonator's radiated sound would be detectable above the background noise, measurements of the Breazeale reactor pool needed to be conducted to characterize the acoustic environment. Once the acoustic environment is characterized, the resonator's detectability can be estimated. This may eventually lead to the monitoring of a nuclear reactor using an acoustic resonator.

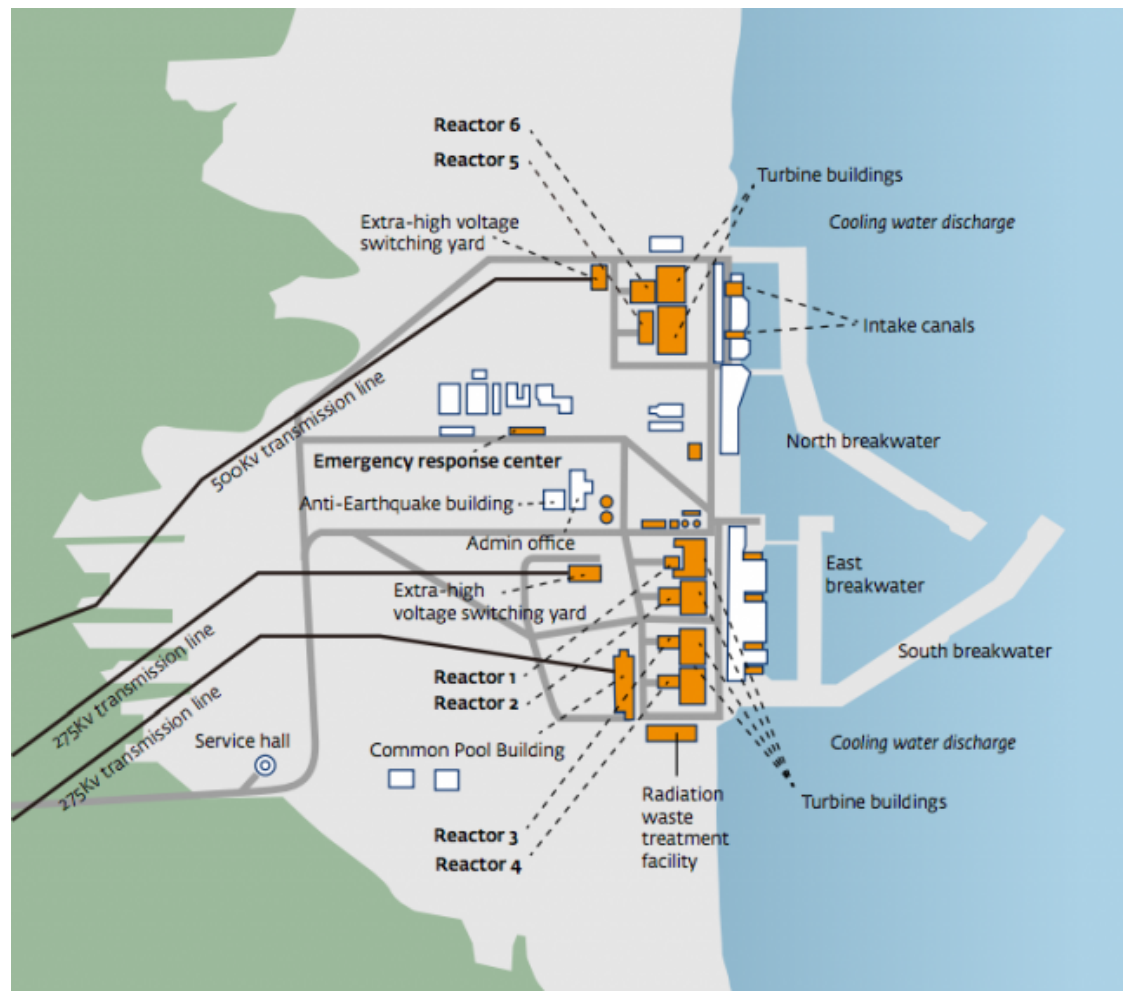

Figure 1.1: Fukushima Daiichi Nuclear Power Plant diagram showing the location of the reactors and the layout of the natural disaster prevention system. The anti-earthquake system, the emergency response center, and the breakwater partitions were constructed to prevent nuclear disasters of the type experienced on March 11, 2011 [12].

\subsection{Background Overview}

The Breazeale Nuclear Reactor is located on the eastern perimeter of Penn State's University Park campus. It is a research reactor capable of producing $1 \mathrm{MW}$ of thermal power. This low-power design (typical commercial reactors can be $500 \mathrm{MW}$ or more [5]) and operating restriction permits the study and testing of nuclear power at the university level without the risks involved with a high-power commercial reactor used by electric utility companies. The Breazeale reactor is a light water reactor cooled by a 70,000 gallon (265 $\mathrm{m}^{3}$ ) pool with a free surface. If the Breazeale reactor were pressurized, the acquisition 
of acoustical data would be more difficult.

If the acoustic resonator is to be powered by nuclear fuel it must first meet the criteria for thermoacoustic engines. The resonator is a hollow cylinder that will be closed at both ends to contain a pressurized gas mixture. It will contain a hot heat-exchanger and a "stack." This type of thermoacoustic engine is called a prime mover, since heat energy (from nuclear fission) is converted to mechanical work (acoustic power) [18]. The half-wavelength standing-wave resonance frequency will be determined by the length and closed-end design of the resonator, along with the gas mixture's sound speed.

The resonance frequency of the thermoacoustic engine will also depend on the appropriately averaged temperature of the gas mixture. Randall Ali and Steven Garrett, at the Pennsylvania State University, investigated the effects of a thermoacoustic resonator submerged in a calorimeter. These experiments conducted by Ali and Garrett, also with support from Idaho National Laboratory, involved the manufacture and testing of the thermoacoustic resonator shown in Fig. 1.2 [2]. The resonator in Ali's thesis used an electric heating element instead of nuclear fuel. A frequency vs. temperature relation was obtained. The results confirmed the resonator's ability to encode the average temperature of the surrounding water as the resonance frequency [3]. Westinghouse Electric Company then suggested that the amplitude of the sound radiated by the thermoacoustic resonator could also be correlated to the flux of energetic particles (i.e. neutrons or gamma rays) [8] [9]. Since the neutron flux of a nuclear reactor is related to its power output, the acoustic amplitude can be used to monitor reactor power. The Breazeale Nuclear Reactor proved to be the perfect initial test site for Westinghouse, Idaho National Lab, and Penn State. Acoustic characterization of the reactor pool was necessary for determining the feasibility of detecting sound generated by the fuel rod resonator.

An electrically driven underwater sound source acted as a mock resonator by emitting various acoustic tones, while hydrophones and an accelerometer picked up the response of acoustic excitation in the reactor pool. This method of excitation and detection was shown to be suitable for these studies in an environment as complex as a nuclear reactor.

\subsection{Thesis Scope}

The primary function of this thesis is to act as a bridge between the research conducted by Ali and Garrett using an electrically heated engine and the future work involving the placement of a TAC sensor in the core of a nuclear reactor. The thesis consists of four parts: theoretical calculations, experimentation, comparison of theory and measurements, and a summary followed by a discussion of future work. The acoustic environment of the reactor pool is the focus of this work. A nuclear was developed by Penn State and IST Mirion [1], and was successfully tested in the Breazeale Reactor Pool; however, publication of those measurements will be published after the completion of this thesis.

First, the shape of the Breazeale reactor pool is described. A few assumptions are made 


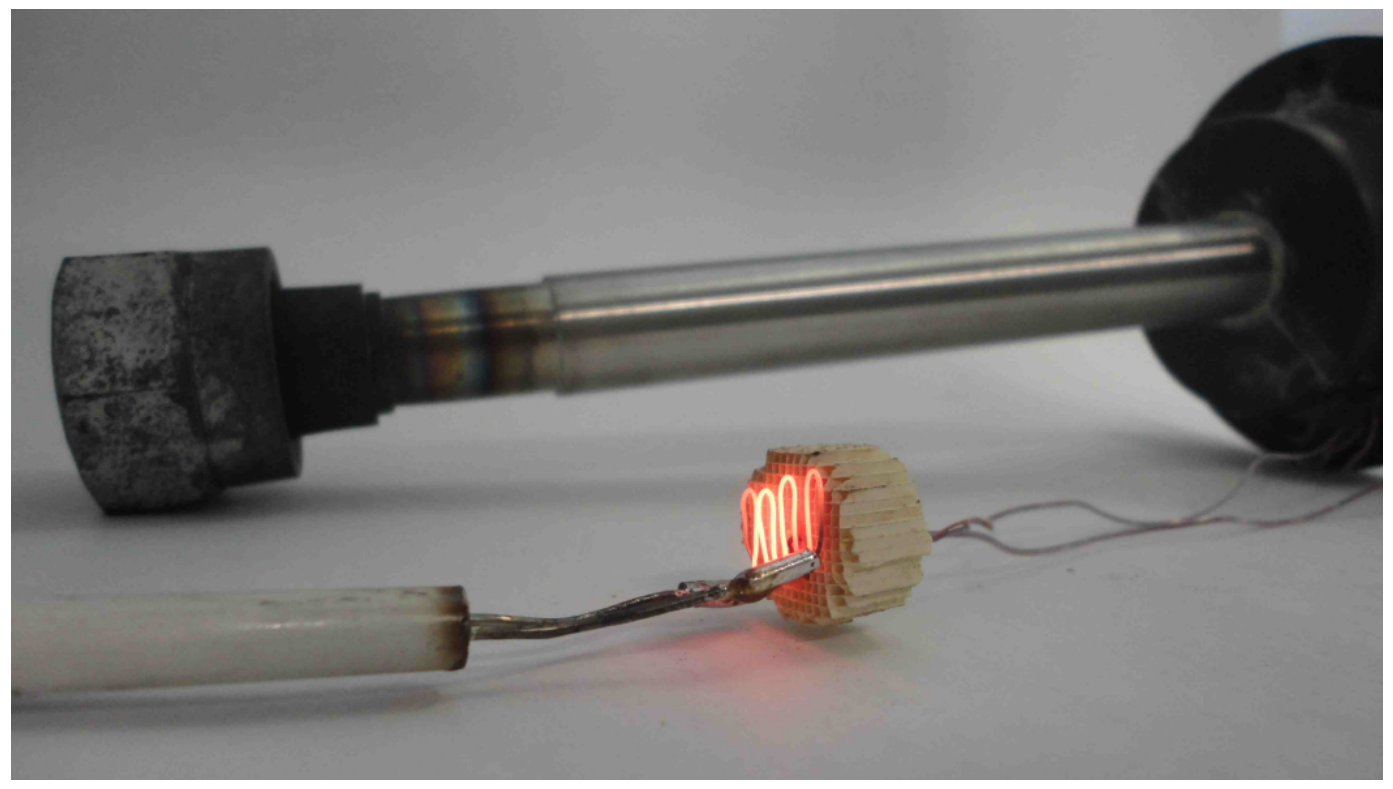

Figure 1.2: Photo of the resonator used for Ali and Garrett's experiments [2]. The heating element is the $\mathrm{NiCr}$ wire glowing in the foreground, and the stack is the larger honeycomblike material to the right of the heater wire. This device is a simplified version of the thermoacoustic fuel rod resonator that will be placed in the Breazeale reactor, but served as the first step in showing the relation between the temperature and frequency of a resonator submerged in water.

to simplify its characterization. Once its geometry is established, the modal character of the space is explored using classical acoustic theory assuming lossless conditions [13]. The pressure-release boundary between the air and water of the reactor pool is important for characterizing not only the modes of the pool, but the ability to monitor acoustic signals in the pool. This pressure-release boundary permits the easy insertion of hydrophones in water, whereas in normal reactors, the pressurization makes this extremely difficult due to potential leakage of irradiated water.

Next, the energy of the acoustic field is related to acoustic losses. Absorption in the reactor pool determines the steady-state acoustic energy. The energy absorbed by the pool walls or transmitted through its boundaries will directly influence the measured sound pressure levels produced by the resonator within the pool. Measurements of values such as reverberation time are used to quantify those losses.

The assumption of dipolar acoustic radiation from a resonator is also examined in that chapter. The focus in this thesis is not on resonator design, which is detailed in a separate Technical Report by Steven Garrett for Idaho National Lab [21].

The resonator was replaced with an underwater sound source for the vibroacoustic measurements reported in this thesis, and the frequency spectra of the reactor's pump noise was obtained under a variety of reactor operating conditions. In short, the underwater sound source was used as a surrogate fuel rod resonator in the Breazeale reactor 
pool. This permitted the study of the acoustic field in an operating nuclear reactor.

On November 12, 2014 and December 04, 2014, acoustic measurements were taken at Breazeale using two hydrophones and an accelerometer. First, both hydrophones were located on the far end of the reactor pool away from the core. During these measurements, both hydrophones were equally spaced on either side of the underwater sound source. The main reactor pumps were deactivated to acquire background noise levels. Once the background noise was measured, a coolant pump was activated and the measurements were repeated. The coolant pump did not raise the noise floor by much, but it did result in a detectable amount of broadband acoustic energy. Reverberation measurements were taken while the coolant pump was deactivated to determine the absorptive behavior of the walls of the reactor pool. The measurements showed that the hydrophones were placed too close to the underwater sound source (see Sec. 3.1.4).

After realizing that the hydrophones were picking up direct instead of reverberant energy, Hydrophone-008 was relocated across the pool. During the subsequent series of measurements (taken December 04, 2014) one hydrophone, Hydrophone-007, was about $6 \mathrm{ft}$. $(1.83 \mathrm{~m})$ from the sound source and the other, Hydrophone-008, was roughly $27 \mathrm{ft}$. (8.2 m) from the sound source. Upon reconfiguration of the hydrophone setup, reverberation measurements were retaken and yielded useful results. The reverberation measurements, in combination with energy absorption theory of the reactor pool, determine the necessary radiated acoustic power output needed to produce pressure amplitudes that could be detected over the background noise in the pool.

The radiated frequency of the fuel rod resonator is determined by the temperature of the gas in the resonator, and consequently the temperature of the water in the pool. With this in mind, measurements of various frequencies were taken. A frequency sweep was used to mimic frequency excursions of the resonator. With high signal-to-noise ratio, this experiment showed positive results after signal processing of the recorded signals. Similar signal processing showed that several frequencies are able to be distinguished simultaneously, proving that multiple resonators could be placed in a reactor core. This frequency-division multiplexing assumes that each resonator's frequency is spaced sufficiently far from any other resonator's frequency.

On November 12th and December 4th, recordings were made of single frequency, multifrequency, and frequency sweep excitations. Later analyses were conducted with the intention of developing software for characterizing frequency and amplitude data taken from a thermoacoustic resonator placed within the Breazeale reactor pool. Section 3.3 focuses on post-acquisition analysis of measured acoustic signals. When the resonator begins emitting sound, signal processing methods will be of paramount importance during the real-time analysis of the received signals to ensure accurate understanding of the resonator and how its properties, i.e. frequency and amplitude, relate to what is happening in the surrounding nuclear environment. Several filtering techniques are explored in this thesis, as well 
as the need for a two-factor analysis scheme for characterizing the resonator's frequency and amplitude.

A data acquisition system must be able to measure frequency and amplitude continuously. This is why the spectrogram and power spectrum are important for monitoring the frequency and amplitude content. The necessity for both analysis tools is discussed in detail in Sec. 3.3.3. A waterfall plot is also mentioned as a possible solution to the spectrogram/power spectrum requirement, however, it is not fully explored. The signal processing in this thesis is limited to the detectability of the signals emitted by the underwater sound source. Windowing, averaging, and overlap methods were all used to produce most of the plots provided in this thesis. It is likely that more complex methods of filtering, windowing, and processing methods can be developed, if required.

Chapter 4 uses the measurements and theory to characterize a fuel rod resonator. Signal processing methods are also being developed by Idaho National Laboratory. The conclusions made in the final chapter focus on the success of the vibroacoustic characterization of the Breazeale reactor using assumptions about the pool. 


\section{Chapter 2 \\ Reactor Pool Sound Field Analysis}

\subsection{Reactor Pool Geometry}

Acoustic characterization of any environment requires certain generalizations to be made about the behavior of sound in both the given geometry and at the frequencies of interest. In the case of the Breazeale Nuclear Reactor pool, sensor frequencies were chosen such that their wavelengths in water were much smaller than any given dimension of the pool. This analysis begins with calculation of the lowest 100 standing wave modes. At the frequencies of interest, the density of modes becomes sufficiently high that the statistical energy analysis of Sabine is useful [19]. Geometry, frequency, and energy simplifications, together provide a better understanding how an acoustic environment behaves during excitation. In the following calculations, these techniques will be used to characterize the sound field within a rigid-walled rectangular pool with a free surface at the air-water interface that is excited by a single dipolar source.

\subsubsection{Architectural Overview}

The Breazeale reactor pool (BRP) has a 2-D hexagonal floor plan and an inner geometry that contains a partition and several other obstructions. The top of the pool is a pressure-release surface (free), while the other six sides and bottom are composed of steelreinforced concrete. The low acoustic impedance of the free surface eliminates acoustic pressure oscillations at the water-air interface. Figure 2.1 shows a simplified model of the reactor pool's geometry.

The BRP is approximately $14 \mathrm{ft}(4.27 \mathrm{~m})$ wide and $30 \mathrm{ft}(9.14 \mathrm{~m})$ long. $24 \mathrm{ft}(7.32 \mathrm{~m})$ of depth gives an overall volume of more than 70,000 gallons $(265 \mathrm{~kL})$, this volume provides an ample amount of water for cooling the reactor during normal operation. The pool consists of a north and a south bay, connected through a $5 \mathrm{ft}(1.5 \mathrm{~m})$ wide opening. The north bay is considered the storage or drainage section, while the south bay acts as the primary operation area. All of the walls and partitions, with the exception of the southernmost wall labeled -4- in Fig. 2.1, are made of $1.5 \mathrm{ft}(.46 \mathrm{~m})$ thick concrete. The south wall (wall 4) is reinforced by $3.5 \mathrm{ft}(1.07 \mathrm{~m})$ thick high-density concrete to shield the neutron beam

ports next to the reactor. A removable aluminum gate that can be inserted between the 


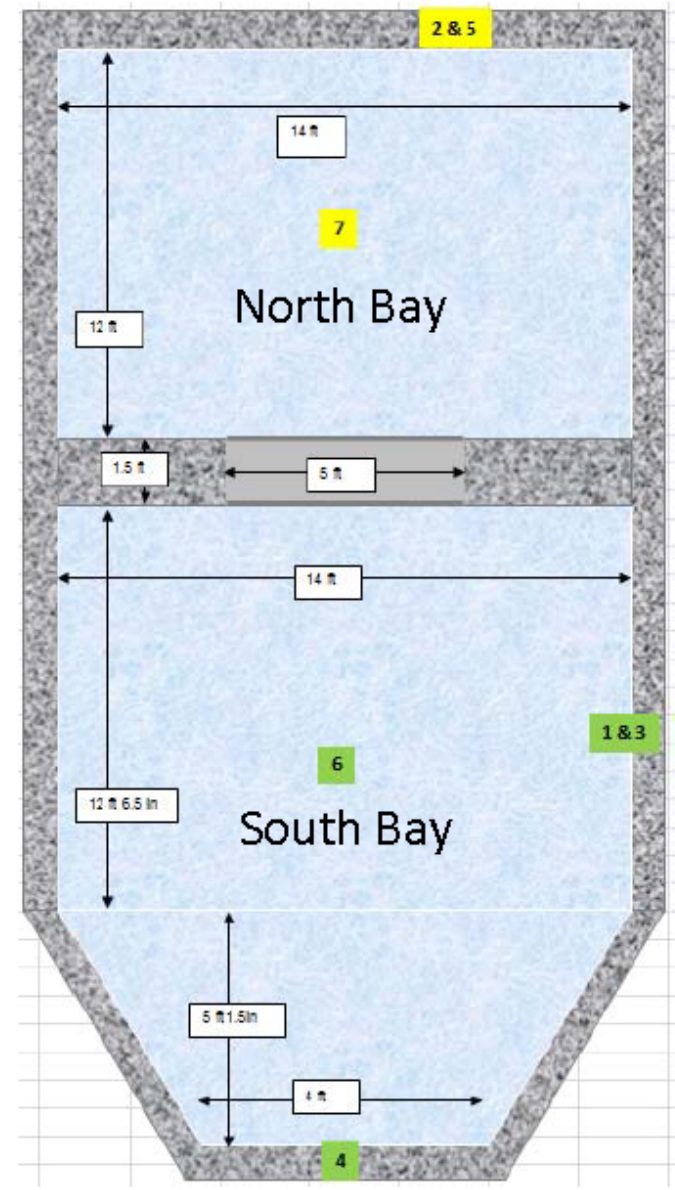

Figure 2.1: Simple geometric representation of the Breazeale Nuclear Reactor pool. The depth of the pool is $24 \mathrm{ft}(7.32 \mathrm{~m})$ and its sides are made of steel-reinforced concrete $1.5 \mathrm{ft}(0.46 \mathrm{~m})$ thick. The southernmost wall located in the south bay, labeled $-4-$, is $3.5 \mathrm{ft}(1.07 \mathrm{~m})$ thick to shield the neutron beam ports. Typically, the core is located near the center of the south bay, labeled $-6-$.

north and south bays in the $5 \mathrm{ft}(1.5 \mathrm{~m})$ gap that divides the two. The gate is usually absent to allow the two bays to mix. That gap is treated as open for the calculations that follow.

\subsubsection{Geometric Assumptions}

As a first step toward simplification of the acoustic environment, its partly hexagonal floor plan is approximated as rectangular with the same cross-sectional area. Using the measurements from Fig. 2.1, the approximate area of the $5 \mathrm{ft}(1.5 \mathrm{~m})$ gap between the two pools is $7.5 \mathrm{ft}^{2}\left(.7 \mathrm{~m}^{2}\right)$, and the area of the irregular polygon in the south pool is $71.8 \mathrm{ft}^{2}$ $\left(6.7 \mathrm{~m}^{2}\right)$. Figure 2.2 depicts the resulting adiabatic transformation from irregular to rectangular area (labeled A to B) [7]. Sections in red denote changes, while all other dimensions remain unchanged. Since the depth is unaltered and the overall area remains the same $\left(397 \mathrm{ft}^{2}\left(36.9 \mathrm{~m}^{2}\right)\right)$, the volume is conserved as well. Drawing B in Fig. 2.2 is the working model for acoustic analysis of the Breazeale reactor pool that follows. 

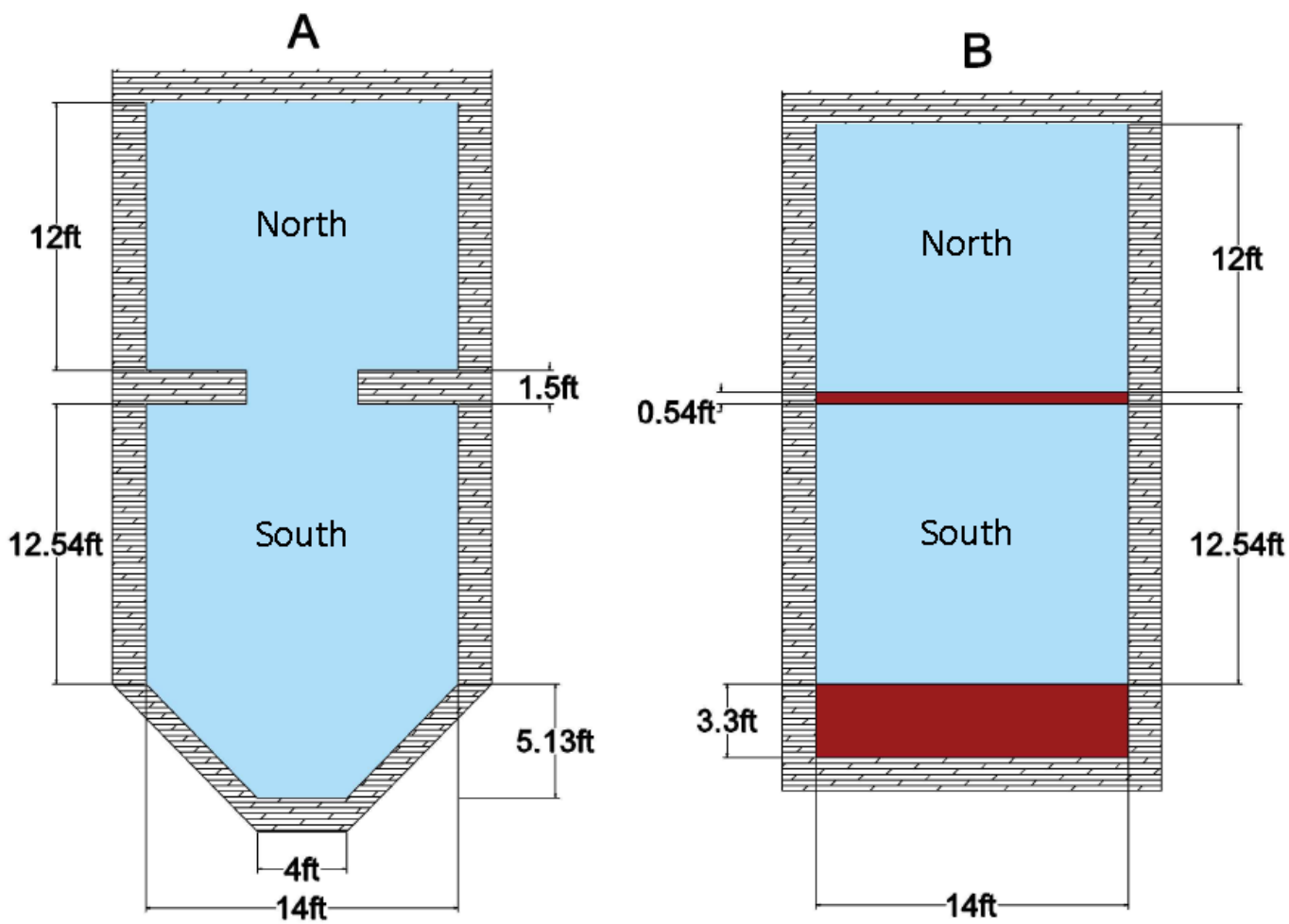

Figure 2.2: Simple geometric representation showing the adiabatic transformation from hexagonal to rectangular floor plan.

\subsection{Identifying a Suitable Frequency Band}

The Breazeale reactor pool (BRP), with its simplified geometry represented in Fig. 2.2(B), is treated as a "rectangular room." Furthermore, approximations and assumptions must be made about the acoustic environment with regard to propagation of sound in water. A perfect pressure-release surface is assumed for the BRP analysis. Absence of thermal, viscous, relaxation, transmission, and scattering energy losses are also assumed in the water and at the pool boundaries. These assumptions will later be modified to include losses, but their immediate neglect does not invalidate the derivations that follow. Lastly, it is assumed that the steel-reinforced concrete walls are sufficiently rigid that ideal standingwaves are formed with no normal fluid velocity at any boundary except the free surface. Through superposition of the aforementioned requirements, modal frequency information can be recovered for the desired geometry, therefore, facilitating further analysis of the acoustic environment [6] [15].

\subsubsection{Rigid and Pressure-Release Boundaries}

For the reactor pool, interpretation of boundary conditions and modal behavior requires that a three-dimensional wave equation be solved. To begin, $p$ is designated as the acoustic 
pressure. It is a function of position and time. $c$ is a constant defined as the speed of sound in the fluid. The spatial and temporal variation of $p$ is governed by the linearized, non-dissipative wave equation:

$$
\nabla^{2} p=\frac{1}{c^{2}} \frac{\partial^{2} p}{\partial t^{2}}
$$

Equation 2.1 can be expressed in rectangular coordinates:

$$
\frac{\partial^{2} p}{\partial x^{2}}+\frac{\partial^{2} p}{\partial y^{2}}+\frac{\partial^{2} p}{\partial z^{2}}=\frac{1}{c^{2}} \frac{\partial^{2} p}{\partial t^{2}}
$$

Since $p$ is a function of $x, y, z$, and $t$, it can be separated into the product of four separate functions by utilizing the Helmholtz equation, each labeled in terms of its respective coordinate $[15]$.

$$
p(x, y, z, t)=X(x) Y(y) Z(z) T(t)
$$

Plugging Eq. 2.3 back into Eq. 2.1, the solution results in a sinusoidal pressure distribution in terms of position and time (using $i=\sqrt{-1}$ as the unit imaginary number):

$$
\begin{gathered}
p(x, y, z, t)=A_{x, y, z} e^{i\left(k_{x} x+k_{y} y+k_{z} z-\omega t\right)} \\
\omega=c \sqrt{k_{x}^{2}+k_{y}^{2}+k_{z}^{2}}=c|\mathbf{k}|
\end{gathered}
$$

At this point, $A_{x, y, z}$ is the amplitude, $k_{n}$ is defined as a cartesian component of the acoustic wavenumber, and $\omega$ is called the angular frequency: $\omega=c|\mathbf{k}|$. Values of $k_{x}, k_{y}, k_{z}$ are predetermined by the boundary conditions set by the geometry and architecture of the pool. Each modal frequency can then be calculated using the definition of the wavenumber. If the lengths of the walls of the pool are labeled $L_{x}, L_{y}, L_{z}$, respectively for each dimension, then relations between boundary conditions and wavenumbers can be imposed. Due to the rigid-walled assumption, the normal component of the fluid velocity must be identically zero at a rigid boundary (i.e. concrete). Using Euler's equation, a relation between particle velocity and pressure can be established.

$$
\begin{gathered}
\rho \frac{\partial \mathbf{u}}{\partial t}=-\nabla p \\
\mathbf{u}=-\frac{1}{\rho} \int \nabla p d t=\frac{1}{\rho} \frac{k_{n}}{\omega} p=0 \quad \text { [for rigid boundary] } \\
\frac{\partial \mathbf{u}}{\partial t}=-\frac{1}{\rho} \nabla p=-\frac{i k_{n}}{\rho} p=0 \quad \text { [for pressure-release boundary] }
\end{gathered}
$$

A Cartesian coordinate system is assumed, with $x$ and $y$ in the directions parallel to the bottom of the pool, and $z$ in the direction vertically from the bottom of the pool toward the pressure-release surface. The origin of the coordinate system is assumed to be at $x=y=z=0$ at the bottom of the pool where the three perpendicular planes 
intersect. The lengths of the sides of the pool are $L_{x}, L_{y}$, and $L_{z}$, corresponding to its subscripted direction. The actual dimensions calculated previously will be used in the mode calculation, but are not necessary for the derivation.

The $x$ and $y$ pressure solutions are easily found through symmetrically rigid boundaries at $x=y=0$ and $x=L_{x}, y=L_{y}$. The $z$-direction is different because it requires a rigid surface at $z=0$, but a pressure released surface at $z=L_{z}$. Eqs. 2.7 and 2.8 for $z$-direction can be solved using the solution from Eq. 2.4:

$$
\begin{gathered}
p(x, y, z, t)=A_{x, y, z} e^{i\left(k_{x} x+k_{y} y+k_{z} z\right)} e^{-i \omega t} \quad \rightarrow \quad \mathbf{u}=\frac{1}{\rho} \frac{k_{n}}{\omega} p=0 \quad \text { [rigid boundary] } \\
p(x, y, z, t)=A_{x, y, z} e^{i\left(k_{x} x+k_{y} y+k_{z} z\right)} e^{-i \omega t} \quad \rightarrow \quad \frac{\partial \mathbf{u}}{\partial t}=-\frac{i k_{n}}{\rho} p=0 \quad \text { [soft boundary] }
\end{gathered}
$$

Euler's formula is employed using complex numbers to gain intuitive understanding into the behavior of sound waves at each boundary. The real part of each trigonometric function corresponds to the physical phenomena. Although sine or cosine are chosen based on a specified boundary, both must be incorporated for the complete solution [13]. The results derived for the $z$-direction are shown below using the sine function.

$$
\begin{aligned}
\mathbf{u}(0,0,0, t) & =\frac{1}{\rho} \frac{k_{z}}{\omega} p(0,0,0, t)=\frac{1}{\rho} \frac{k_{z}}{\omega} \sin \left(k_{z} \cdot 0\right)=0 \\
\mathbf{u}\left(0,0, L_{z}, t\right) & =-\frac{i k_{z}}{\rho} p\left(0,0, L_{z}, t\right)=\frac{k_{z}}{\rho} \cos \left(k_{z} L_{z}\right)=0
\end{aligned}
$$

Equation 2.11 indicates that for all values of $k_{z}$ the boundary condition is met. Equation 2.12, however, requires all values of $k_{z} L_{z}$ be an odd multiple of one-half multiplied by $\pi$.

$$
\begin{gathered}
k_{z}=\frac{\left(2 n_{z}-1\right) \pi}{2 L_{z}} \\
f_{z}=\frac{c}{2 \pi} \frac{\left(2 n_{z}-1\right) \pi}{2 L_{z}}=\frac{c}{4 L_{z}}\left(2 n_{z}-1\right) \\
\left(n_{z}=1,2,3,4 \ldots\right)
\end{gathered}
$$

This accounts for the modes in the $z$-direction. Now, the same technique must be used to find the modes in the $x$ and $y$ directions.

$$
k_{x}=\frac{n_{x} \pi}{L_{x}}, \quad k_{y}=\frac{n_{y} \pi}{L_{y}}
$$




$$
\begin{gathered}
f_{x}=\frac{c}{2 L_{x}} n_{x}, \quad f_{y}=\frac{c}{2 L_{y}} n_{y} \\
\left(n_{x}, n_{y}=0,1,2,3 \ldots\right)
\end{gathered}
$$

Invoking the definition of $\omega$ from Eq. 2.5, $k_{x}, k_{y}$ and $k_{z}$ from Eqs. 2.15 and 2.13, and the relation $\omega=2 \pi f$,

$$
f_{n_{x}, n_{y}, n_{z}}=\frac{c}{2} \sqrt{\left(\frac{n_{x}}{L_{x}}\right)^{2}+\left(\frac{n_{y}}{L_{y}}\right)^{2}+\left(\frac{\left(2 n_{z}-1\right)}{2 L_{z}}\right)^{2}}
$$

This is an important result that relates the geometry of a rectangular enclosure to the standing-wave frequencies produced during steady-state excitation. Each value for $n_{x}, n_{y}$ and $n_{z}$ will produce a modal frequency for the given geometry.

\subsubsection{Modal Characteristics of the Pool}

Implementation of Eq. 2.17 begins with substitution of allowed integer values for $n_{x}, n_{y}$, and $n_{z}$. The speed of sound in fresh water is $c \simeq 1500 \mathrm{~m} / \mathrm{s}$ (at $25^{\circ} \mathrm{C}$ ), the lengths of the sides of the pool given in Fig. $2.2 \mathrm{~B}$ are: $L_{x}=8.65 \mathrm{~m}, L_{y}=4.27 \mathrm{~m}$, and $L_{z}=7.32 \mathrm{~m}$. The nine lowest frequency modes of the BRP are displayed in Table 2.1 with their corresponding integer indices.

\begin{tabular}{|c||c|c|c|c|c|c|c|c|c|}
\hline \multicolumn{10}{|c|}{ Reactor Pool Modal Frequencies } \\
\hline & $f_{0,0,1}$ & $f_{1,0,1}$ & $f_{0,0,2}$ & $f_{1,0,2}$ & $f_{2,0,1}$ & $f_{0,1,1}$ & $f_{1,1,1}$ & $f_{2,0,2}$ & $f_{0,1,2}$ \\
\hline \hline$n_{x}$ & 0 & 1 & 0 & 1 & 0 & 1 & 1 & 2 & 0 \\
\hline$n_{y}$ & 0 & 0 & 0 & 0 & 1 & 1 & 1 & 0 & 1 \\
\hline$n_{z}$ & 1 & 1 & 2 & 2 & 1 & 1 & 1 & 2 & 2 \\
\hline Frequency $[\mathrm{Hz}]$ & 51 & 101 & 154 & 177 & 181 & 183 & 203 & 232 & 234 \\
\hline
\end{tabular}

Table 2.1: First nine modal frequencies of the Breazeale reactor pool. Three types of modes are demonstrated: axial (only one non-zero index), tangential (only one zero index), and oblique (no zero indices). Mode types are important for accurate room acoustic calculations in spaces inhabited by humans to establish perception and hearing, however, the current analysis does not necessitate distinction between them.

The frequency separation between neighboring peaks decreases as the indices increase, thus resulting in a larger density of modes at higher frequencies. At sufficiently high frequencies, a statistical theory of energy distribution can be used to characterize properties of reverberant spaces. The total volume, $V$, surface area, $S$, and volumetric perimeter, $L_{t o t}$, are used to approximate the number of modes, $N$, in a room below a particular frequency, $f$ [14]. 


$$
N \simeq\left(\frac{4 \pi f^{3} V}{3 c^{3}}\right)+\left(\frac{\pi f^{2} S}{4 c^{2}}\right)+\left(\frac{f L_{t o t}}{8 c}\right)
$$

For the Breazeale reactor pool, the following values are used:

$$
V \simeq 267 \mathrm{~m}^{3} ; \quad S \simeq 260.5 \mathrm{~m}^{2} ; \quad L_{t o t} \simeq 80.5 \mathrm{~m}
$$

Using this approach, the number of modes below $1 \mathrm{kHz}$ is approximately 433 for the BRP geometry. Using a simple MATLAB ${ }^{\circledR}$ routine, 408 modes were calculated, putting the approximation within $6 \%$ of the exact result. The discrete values and Eq. 2.18 converge as the frequency increases, causing the error to decrease.

Eq. 2.18 can also be used to calculate the density of modes within a specified bandwidth, $d f$, by taking the derivative with respect to $f$ :

$$
\frac{d N}{d f} \simeq\left(\frac{4 \pi f^{2} V}{c^{3}}\right)+\left(\frac{\pi f S}{2 c^{2}}\right)+\left(\frac{L_{t o t}}{8 c}\right)
$$

Eq. 2.19 is solved as a quadratic equation for $f$. For example, if the goal was to find the frequency where 10 modes per $1 \mathrm{~Hz}$ bandwidth existed, then the modal density can be set to $d N / d f=10$. In that case, one would arrive at a frequency around $3,063 \mathrm{~Hz}$, using the dimensions of the Breazeale pool. A quick check in MATLAB ${ }^{\circledR}$ confirms that around 3,063 Hz there are roughly 10 modes within each $1 \mathrm{~Hz}$ bandwidth.

Consideration of the modal density permits a smooth transition from frequency to energy considerations in a reverberant environment. Manfred Schroeder suggested a cutoff frequency, later renamed the eponymous "Schroeder frquency," that marks the transition between discrete resonance frequencies with resolvable peaks and resonant modes that overlap sufficiently that they can be modeled statistically. This cutoff frequency can also be used in conjunction with energy analysis to characterize the acoustic energy in the reactor pool [20]. Based on empirical observations, Schroeder defined his cutoff frequency as the frequency where three modes exist within the $-3 \mathrm{~dB}$ bandwidth of a single mode.

\subsection{Energy in the Acoustic Environment}

Energy losses through walls play an important role in the characterization of dissipation throughout the reactor pool's acoustic environment. The inclusion of these losses is the next logical step in the modal analysis, and will eventually connect experimental values with the steady-state acoustical energy distribution. The geometric and pressure-release assumptions still hold, however, the rigid-walled approximation no longer holds due to non-zero absorption and transmission mechanisms at the boundaries. For the Breazeale reactor pool, bulk thermoviscous losses are negligible because of the frequencies of interest and the short distances traveled by the acoustic waves. This will be covered in more detail in the next section. 
An enclosure can be thought of as a series of small resonators excited by a source, each relating to a standing wave frequency that will decay once the source is turned off. This behavior can be analyzed in the same way that statistical mechanics relates to classical mechanics. In classical mechanics, it is assumed that large bodies follow conservation laws individually, based on specific properties, whereas statistical mechanics relies on the grouping of large quantities of particles that produce statistical behavior. An example of this is frequently seen in acoustics with the treatment of air as a large group of particles moving together to create a wave. An extension of this analogy is applied to a different phenomenon in acoustics where the equal distribution of energy among modes that are very closely spaced in frequency treat modes as individual particles.

\subsubsection{Conserving Energy through Absorption}

The power density associated with an acoustic wave is often expressed as a sum of the time derivative of the kinetic and potential energy densities and the divergence of a flux. In acoustics, this energy flux is the acoustic intensity, I [15]:

$$
\frac{\partial w}{\partial t}+\nabla \cdot \mathbf{I}=0
$$

Integrating Eq. 2.20 over a specified volume gives the total power for sources emitting a band of frequencies:

$$
\begin{gathered}
\frac{d}{d t} \iiint w_{s} d V=\Pi_{s}-\Pi_{s, d} \\
w_{s}=\frac{1}{2} \rho_{0} v_{s}^{2}+\frac{1}{2} \frac{p_{s}^{2}}{\rho_{0} c^{2}} ; \quad \mathbf{I}=p \mathbf{v}_{\mathbf{s}}
\end{gathered}
$$

The variables $p_{s}$ and $\mathbf{v}_{\mathbf{s}}$ are functions of $\mathbf{x}$ and $t$ and represent source conditions. $\Pi_{s}$ and $\Pi_{s, d}$ are the radiated and dissipated power of the source, respectively, and $w_{s}$ describes the mechanical energy density of the system produced by the sources.

Time average functions of $\Pi_{s}, \Pi_{s, d}$, and $w_{s}$ are represented as $\bar{\Pi}, \bar{\Pi}_{d}$, and $\bar{w}$, respectively, and require that the values of each average be independent of location. This stems from Sabine's statistical analysis of reverberant rooms, which requires that any wavelength be much smaller than any fundamental dimension of the enclosure. The averaged "dissipated power," $\bar{\Pi}_{d}$, can be approximated by assuming all energy loss is due to transmission through the boundaries.

$$
\bar{\Pi}_{d}=\frac{c}{4} A_{a b s} \bar{w}
$$

Equation 2.23 relates the absorption of sound in a room to the energy produced by the source, $\bar{w}$, through an equivalent absorptive area, $A_{a b s}$. Substituting Eq. 2.23 into Eq. 2.21 results in a differential equation relating the average energy output, average power 
output, and absorptive area at the enclosure's boundaries:

$$
\begin{gathered}
\frac{d}{d t} \iiint \bar{w} d V=\bar{\Pi}-\frac{c}{4} A_{a b s} \bar{w} \\
\bar{\Pi}=V \frac{d \bar{w}}{d t}+\frac{c}{4} A_{a b s} \bar{w}
\end{gathered}
$$

Equation 2.25 states that the total power of a source will be broken into the sum of the average energy density and the absorption proportional to the average energy density in the system. Upon cessation of the source output $(\bar{\Pi}=0)$, the differential equation in 2.25 can be solved using a simple exponential decay solution:

$$
\begin{gathered}
\int \frac{1}{\bar{w}} d \bar{w}=\int-\frac{c}{4 V} A_{a b s} d t \\
\ln \left(\frac{\bar{w}}{\bar{w}_{0}}\right)=-\frac{c}{4 V} A_{a b s} t \rightarrow \bar{w}=\bar{w}_{0} e^{-t / \tau} \\
\tau=\frac{4 V}{c A_{a b s}}
\end{gathered}
$$

Equation 2.28 is often used to calculate the time it takes for the energy in a system to decrease by 60 decibels. This $60 \mathrm{~dB}$ decrease was chosen by Sabine as the time it took for sound in a room to decay to an inaudible level.

$$
\begin{gathered}
\text { decibel }=10 \log _{10}\left(\frac{p_{\text {rms }}^{2}}{p_{\text {ref }^{2}}^{2}}\right) \\
-60=10 \log _{10}\left(\frac{\bar{w}_{60}}{\bar{w}_{0}}\right) \rightarrow \bar{w}_{60}=\bar{w}_{0} 10^{-6}
\end{gathered}
$$

The specific subscripted $\bar{w}_{60}$ is designated as the 60 decibel decay from the original power level. The insertion of $\bar{w}_{60}$ and $\bar{w}_{0}$ as squared-pressures stems from the definition of the energy density in Eq. 2.22 and its dependence on the pressure, $p$. Through Euler's equation (Eq. 2.6), the velocity in Eq. 2.22 can also be written in terms of the pressure, if sinusoidal sources are assumed, as is the case here. Now if the second of Eq. 2.30 is used to calculate a value for $t$, the result is widely recognized as the "reverberation time," $T_{60}$, and can be determined through experimentation.

$$
\begin{gathered}
e^{t / \tau}=10^{6} \rightarrow t=6 \ln (10) \tau \\
T_{60}=6 \ln (10) \tau=13.82 \tau
\end{gathered}
$$

Equations 2.32 and 2.28 use the time it takes for a signal's power to decrease by 60 decibels to infer the effective absorptive area of an enclosure. It is often used to character- 


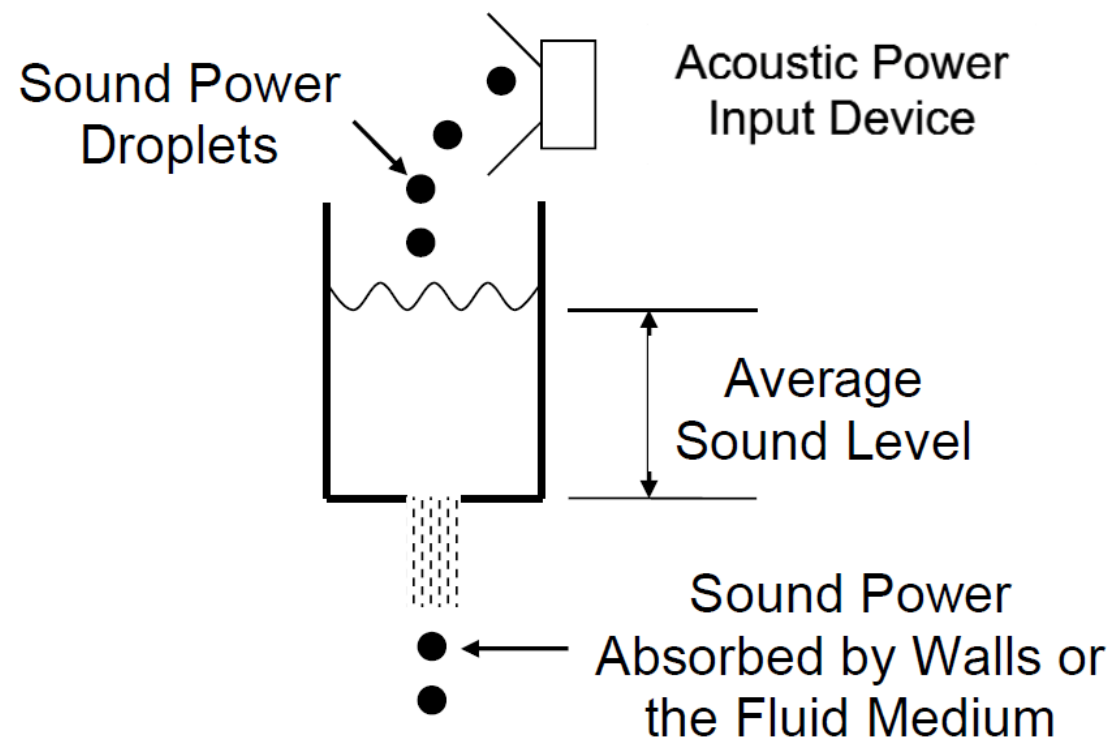

Figure 2.3: Illustration showing the effects of absorption on a system. The power output is a constant sound source emitting 'sound power droplets' into a bucket that represents the acoustic environment. An average sound level is produced, based on an average absorption. The power absorbed and the average measured sound power add to equal the total acoustic power input [21].

ize the clarity of speech in a room, but more importantly in our case, it helps determine how quickly energy escapes from an enclosure. Figure 2.3 is a cartoon that depicts an acoustic system, such as the Breazeale reactor pool, and simplifies the concepts of power output, average sound level, and average absorption. It also prompts a discussion of the steady-state level.

A method for calculating the time average of two complex variables is generally given by the relation:

$$
(U V)_{a v}=\frac{1}{2} \operatorname{Re}\left\{U V^{*}\right\}
$$

Eq. 2.33 is an identity for the time-averaged product of two complex variables, $U$ and $V$. As previously mentioned in Eq. 2.22, $w_{s}$ contains kinetic and potential energy densities. If assumptions are made about the pressure waveform, namely, that they are sinusoidal in space and time (see Eq. 2.4), then the kinetic and potential energy densities are equal when averaged over an integer number of cycles [15].

$$
\bar{w}=\frac{1}{4} \rho_{0} v v^{*}+\frac{1}{4} \frac{p p^{*}}{\rho_{0} c^{2}}=\frac{1}{2} \frac{|p|^{2}}{\rho_{0} c^{2}} \approx \frac{\overline{p_{r m s}^{2}}}{\rho_{0} c^{2}}
$$

Figure 2.3 illustrates the three mechanisms that determine the steady-state amplitude in an acoustic enclosure: A calibrated output power, an average sound power level, and 
an absorbing power. A microphone, or hydrophone in the case of water, is typically used to measure a root-mean-square pressure $\left(p_{r m s}\right)$ within the enclosure. The rms pressure is then squared to give an average sound power level using Eq. 2.34 and 2.25. The absorption power is then calculated from the subtraction of these two to give an average absorption in the system.

For water, bulk thermoviscous absorption exhibits a quadratic frequency dependence:

$$
\alpha_{t v}=\frac{\omega^{2} \nu}{2 c_{0}^{3}}\left[\left(\frac{4}{3}+\frac{\mu_{B}}{\mu}\right)+\frac{\gamma-1}{P r}\right]
$$

In pure water, the bulk viscosity, $\mu_{B}$, can be ignored along with thermal losses since $\gamma \simeq 1$. The kinematic viscosity is $\nu$ and $c_{0}$ is the sound speed here. Using experimental values for $\nu$ and $c_{0}$ result in a compact equation solely in terms of frequency. For sound attenuation in fresh water at $20^{\circ} \mathrm{C}$, the bulk thermoviscous absorption coefficient can be written as[4]:

$$
\alpha_{t v} \approx 2.17 \times 10^{-13} f^{2} \mathrm{~dB} / \mathrm{m}
$$

For the BRP, the maximum length of any side is $8.54 \mathrm{~m}$. Therefore, if each pressure wave travels two lengths of the pool, and there is only $0.1 \mathrm{~dB}$ of attenuation over that distance, the lowest frequency to achieve that amount of loss is approximately $164 \mathrm{kHz}$. Accordingly, frequencies below $160 \mathrm{kHz}$ experience less than $0.1 \mathrm{~dB}$ of loss over two lengths of the longest side of the pool. And at frequencies below $20 \mathrm{kHz}$ in fresh water at $20^{\circ} \mathrm{C}$, thermoviscous attenuation can be entirely neglected [4].

Conversely, sound waves in large enclosures filled with water experience dissipation governed primarily by wall losses. Equation 2.23 defines the equivalent absorptive area, $A_{a b s}$, as a ratio of the average power dissipated to the average energy in a system. $A_{a b s}$ has units of area, and is treated as a sum of all absorptive surfaces within the given geometry.

$$
A_{a b s}=\sum_{i} \alpha_{i} A_{i}
$$

The variable $\alpha_{i}$ in Eq. 2.37 is usually based on empirical data, but an average overall absorption will be assumed in the following analysis due to the linear nature of the losses at the walls. This will characterize an approximate energy dissipation from all boundaries, and will specify the average energy density in the enclosure. Now, Equations 2.25, 2.34, and 2.37 can combine to give a full expression for the energy in the Breazeale reactor pool:

$$
\bar{\Pi}=\frac{d(\Psi V)}{d t}+\frac{c \Psi}{4} \sum_{i} \alpha_{i} A_{i}
$$

$\Psi$ is defined as the solution to Eq. 2.38, but is simply the potential energy density from Eq. 2.34. Under the assumption that $\bar{p}(0)=0$, an exponential evolution can be determined: 


$$
\begin{aligned}
\Psi(t)=\frac{\overline{p_{r m s}^{2}}}{\rho_{0} c^{2}} & =\frac{4 \bar{\Pi}}{c \sum_{i} \alpha_{i} A_{i}}\left(1-e^{-t / \tau}\right) \\
\tau & =\frac{4 V}{c \sum_{i} \alpha_{i} A_{i}}
\end{aligned}
$$

Pressure amplitude measurements are assumed independent of position because due to averaging conditions stated previously and employed in Eq. 2.34. Several conclusions will be drawn from Eq. 2.39. The result will help formulate a 'diffuse field' analysis of the BRP, which assumes that the energy reaches a steady-state value when time, $t$, surpasses $\tau$ and approaches infinity. Additionally, the equivalent absorptive area of the pool boundaries can be calculated if the sound speed of the medium, the volume of the enclosure, and the exponential decay time, $\tau$, are measured. This method provides a bridge between theoretical calculations and experimental results.

\subsubsection{Steady-State Condition}

Determination of acoustic amplitudes and frequencies within a rectangular enclosure is greatly simplified under the assumption that a 'steady-state' condition has been attained. This steady-state condition assumes all transient disturbances have decayed, and that the average energy over a long period, $t \gg \tau$, remains constant.

$$
\Psi(t=\infty)=\frac{4 \bar{\Pi}}{c \sum_{i} \alpha_{i} A_{i}}
$$

And since $\Psi$ is the average potential energy density from Eq. 2.34, it is easy to relate the acoustic steady-state pressure to the average input power, $\bar{\Pi}$ :

$$
\Psi(t)=\frac{\overline{p_{r m s}^{2}}}{\rho_{0} c^{2}}=\frac{4 \bar{\Pi}}{c \sum_{i} \alpha_{i} A_{i}}\left(1-e^{-t / \tau}\right) \quad \rightarrow \quad p_{\mathrm{rms}}(t=\infty)=\sqrt{\frac{4 \rho_{0} c \bar{\Pi}}{\sum_{i} \alpha_{i} A_{i}}}
$$

Substitution of $\tau$ from Eq. 2.28 results in:

$$
p_{\text {rms }}(t=\infty)=c \sqrt{\frac{\tau \rho_{0}}{V} \bar{\Pi}}
$$

Previously, the variable $\tau$ was calculated from a $60 \mathrm{~dB}$ drop in energy (see Eq. 2.32). This allows Eq. 2.43 to be written as a function of the conventional architectural quantity, $T_{60}$.

$$
p_{\mathrm{rms}}(t=\infty)=c \sqrt{\frac{\rho_{0} T_{60}}{13.82 V} \bar{\Pi}}
$$


Equation 2.44 permits a relation between steady-state pressure amplitude and a known power output and $T_{60}$ measurement; and conversely, an output power can be determined from a measured steady-state pressure amplitude and $T_{60}$ measurement.

Equation 2.44 is also the basis for determining how far a measurement device must be from the source to be in the 'diffuse field.' The critical distance, $r_{d}$, is defined as the distance at which the energy density from a spherically-spreading omni-directional sound source is equal to that of the reverberant energy density. $\Psi_{s}$ is the energy density of the source at a distance $r$, whereas $\Psi_{r}$ is the energy density of the reverberant room derived in Eq. 2.41.

$$
\begin{gathered}
\Psi_{s}=\frac{\bar{\Pi}}{4 \pi r^{2} c}=\Psi_{r}=\frac{T_{60}}{13.82 V} \bar{\Pi} \\
r_{d}=\sqrt{\frac{13.82 V}{4 \pi c T_{60}}}
\end{gathered}
$$

Equation 2.46 designates the minimum distance a measurement device must be placed from a sound source to be in the diffuse sound field and has units of [20].

Section 2.2.2 derived the modal properties of the pool, and specifically, Eq. 2.19 gave insight into the modal density per $1 \mathrm{~Hz}$ bandwidth as a function of frequency. Manfred Schroeder suggested a transition between low and high frequencies where the resolution of frequency peaks changes from easily resolvable to statistically blurred.

Schroeder defines a width between two points that equal half the maximum power of a single frequency peak.

$$
B=\frac{1}{2 \pi \tau}
$$

Equation 2.19 is then used to equate the half-power bandwidth, $B$, to a frequency spacing per mode (the inverse of modal density, see Eq. 2.19). Schroeder suggested that three times the frequency spacing per mode, $\delta f$, could be equated to the $-3 \mathrm{~dB}$ bandwidth of a single mode to obtain an expression for a cutoff frequency. Once above this cutoff frequency, statistical methods would predict the behavior of sound waves emitted from a source.

$$
\begin{gathered}
\delta f \equiv \frac{d f}{d N} \approx \frac{c^{3}}{4 \pi f^{2} V} \\
B=\frac{1}{2 \pi \tau}=3 \delta f=3 \cdot \frac{c^{3}}{4 \pi f^{2} V} \\
f_{c}=c \sqrt{\frac{3 c \tau}{2 V}}=c \sqrt{\frac{3 c T_{60}}{2(13.82) V}}
\end{gathered}
$$


Figure 2.4 demonstrates high modal density within the first 100 modes of the reactor pool. This suggests that the Schroeder frequency will fall below the thermoacoustic resonator's resonant frequency of about $1.6 \mathrm{kHz}$; however, since $\tau$ is inversely proportional to the absorption, the Schroeder frequency could vary. Consequently, it is difficult to determine a Schroeder frequency without some experimentation or previous knowledge of the dissipation mechanisms in a reverberant space.

Garrett also indicates that the Schroeder frequency and the critical distance are interrelated through absorption, and accordingly, both characterize a diffuse environment where acoustic detection and monitoring are not specifically mode dependent [21]. The connection between critical distance and Schroeder frequency is evident upon substitution of the definition of $\tau$ (Eq. 2.40) into the critical distance (Eq. 2.46) as well as the Schroeder frequency (Eq. 2.50).

$$
r_{d}=\sqrt{\frac{\sum_{i} \alpha_{i} A_{i}}{16 \pi}} ; \quad \lambda_{c}=\frac{c}{f_{c}}=\sqrt{\frac{\sum_{i} \alpha_{i} A_{i}}{6}}
$$

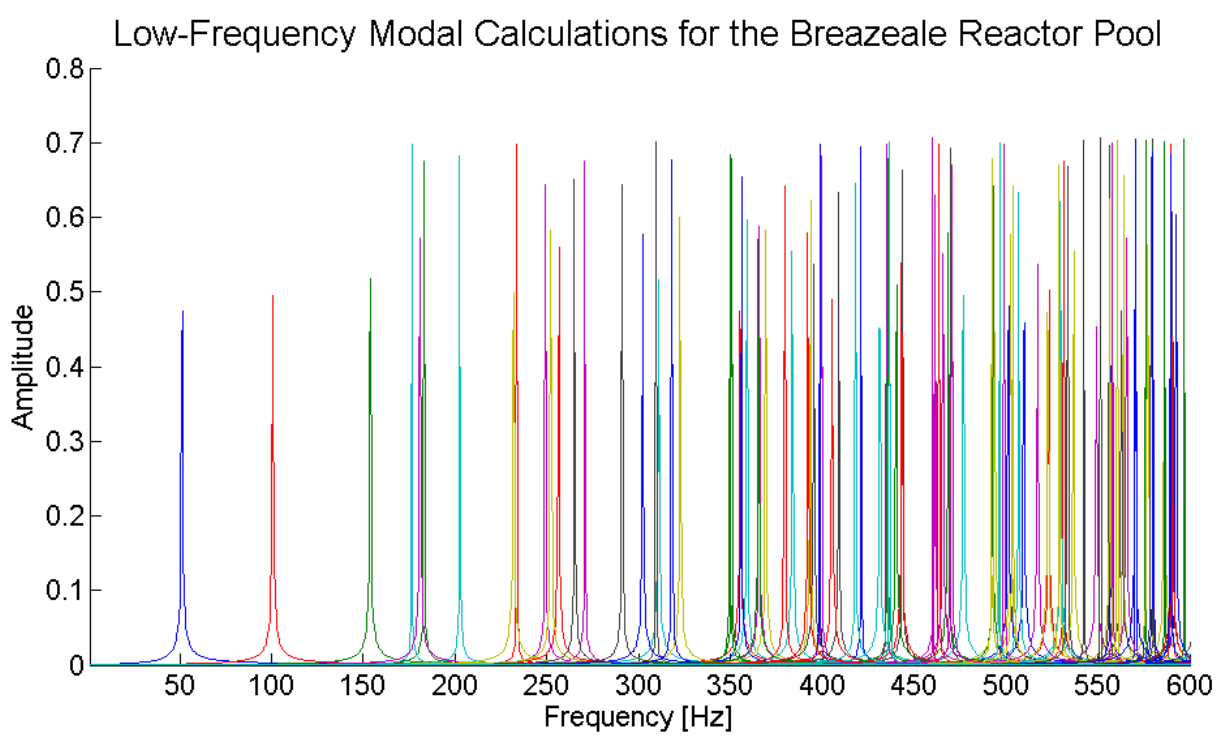

Figure 2.4: First 100 calculated modes of the BRP. The different colors distinguish between adjacent modes and the modal amplitudes are arbitrary. Notice that the modes begin to crowd as early as $180 \mathrm{~Hz}$, and are densely overcrowded by the $500 \mathrm{~Hz}$ mark. For the reactor pool, it is evident that the Schroeder frequency should be lower than the thermoacoustic sensor's resonance frequency.

The connection between the Schroeder frequency and critical distance further determine the frequencies of interest within a given enclosure. And in the case of the BRP, this will help designate an acceptable starting frequency to ensure that the sound source will be emitting frequencies that create a diffuse sound field in the pool. Under this as- 
sumption, the amplitude and frequency will be easy to monitor during temperature and neutron flux measurements.

All of the geometry, frequency, and energy considerations made thus far have contributed to the determination of the diffuse sound field parameters, i.e. critical distance, equivalent absorptive area, and suitable frequency band. The theory thus far has focused on detection of an acoustic sound source placed within the reactor pool, and indicate that information regarding power output and absorption from walls will influence the feasibility of an acoustic resonator used as a sensor within a nuclear reactor. Implementation of such a resonator within a reactor core is limited by sensitivity of materials to radiation that could limit the effectiveness of such a resonator. Below, optimization designs for a nuclear fuel rod resonator are explored.

\subsection{Thermoacoustic Fuel-Rod Resonator}

Now that the acoustic environment of the Breazeale reactor pool has been analyzed from a modal and a reverberant perspective, the feasibility of the proposed thermoacoustic sensor can be explored. The proposed resonator is a form of thermoacoustic engine that functions by converting heat energy to acoustic energy within a given working fluid. There are two types of acoustic heat engines: the prime mover and the heat pump. Heat is added to a prime mover engine, which results in energy flow from hot to cold temperatures. This causes work to be done by the engine. Conversely, work is done on a heat pump, where heat is removed from low temperature and redeposited to higher temperature. Heat pumps are typically called refrigerators, whereas prime movers are generally referred to as heat engines. Appropriately, the thermoacoustic fuel rod resonator is considered a prime mover due to the acoustic work being done by the system as a result of the heat flow [23].

Randall Ali published his Master's thesis in May of 2013 on the fabrication and initial testing of a thermoacoustic sound source. Ali's thermoacoustic sound source was submerged in a small calorimeter filled with water and heated at one end by $\mathrm{NiCr}$ wire. It contained a Celcor ${ }^{\circledR}$ material called a "stack" that functioned as the second thermodynamic medium that could exchange thermal energy with the "working fluid." In Ali's case, the working fluid was air pressurized above atmospheric pressure. The stack material's primary function is to exchange heat with the gas through the four processes illustrated schematically in Fig. 2.5. The four processes are: 1. movement of gas toward the hot end of the stack, accompanied by compression of gas; 2. transfer of heat from the stack to the gas; 3. movement of gas from the hot toward the cold end of stack, accompanied by expansion of gas; 4. transfer of heat from the gas to the stack. The cyclic processes of compression and expansion occur with a phasing that increase the amplitude of the standing wave of the gas inside the resonator [2]. This process was first described by J.W. Strutt, known as Lord Rayleigh, where he explained that heat-maintained acoustic oscillations were governed by the proper phasing of heat transfer and gas compression [17]: 
"If heat be added to the air at the moment of greatest condensation, or be taken from it at the moment of greatest rarefaction, the vibration is encouraged."

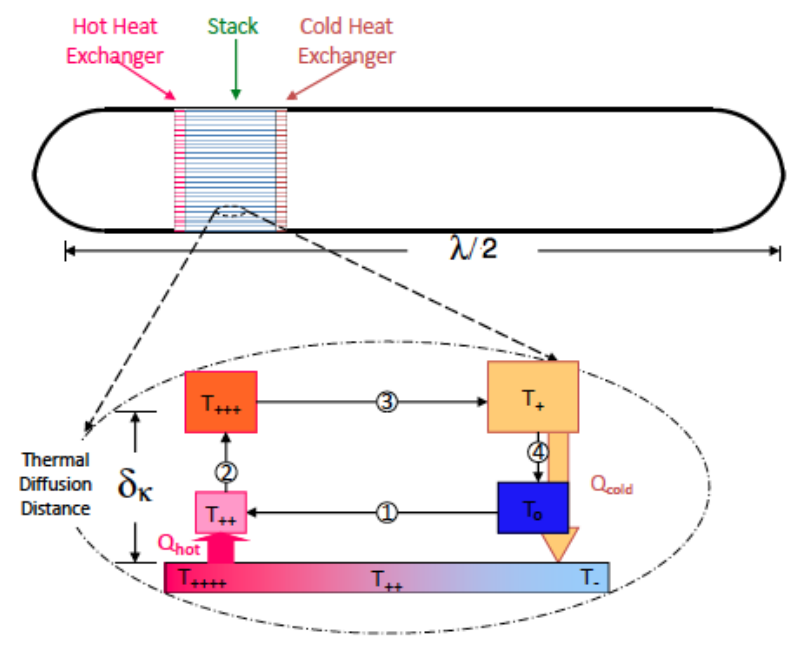

Figure 2.5: Illustration of a half-wavelength resonator that shows a zoomed-in view of the compression and expansion of a gas within the resonator's stack material. Four processes take place within the stack: 1 . movement of gas toward the hot end of the stack, accompanied by compression of gas; 2. transfer of heat from the stack to the gas; 3. movement of gas toward the cold end of stack, accompanied by expansion of gas; 4. transfer of heat from the gas to the stack. The stack is designed so that the pores have gaps only a few thermal diffusion distances, $\delta_{\kappa}$, to permit ample thermal interaction. The heat exchangers promote oscillation by removing and adding heat to the ends of the stack [2].

The results produced by Ali et al. [3] stimulated the interest of acoustic monitoring within a reactor core. His results successfully demonstrated acoustic resonance and the potential for a submerged, heat-driven acoustic sensor to measure the temperature of the surrounding water and encode that information as the frequency of the standing wave. This sensor, photographed in Fig. 1.2, shows the heat supply and stack removed from the resonator. It is a prime mover, closed at both ends, and produces a resonance frequency with a wavelength that is approximately twice the length of the enclosure. The left side of the photo is the hot end of the resonator while the right side is the ambient (cold) end. This closed-closed condition forces a standing-wave resonance based on the geometry of the resonator, the gas, and the temperature of the gas. The gas, stack pore size, stack length, and stack position in the standing wave are parameters that can be optimized to produce the most efficient and dependable resonator.

The oscillatory motion of gas within the resonator causes the entire resonator to move in reaction, and it moves in opposition to the gas motion. The volume of the resonator 
remains constant, but the forces that the gas motion exerts on the resonator can excite dipolar acoustic radiation if the resonator is free to move. Details regarding the dipole radiation of an acoustic resonator experiencing simple harmonic motion are presented in the Idaho National Laboratory Technical Report referenced in the bibliography [21]. 


\section{Chapter 3 \\ Breazeale Reactor Vibroacoustic Measurements}

On November 12th and December 4th of 2014, experiments were conducted to acoustically characterize the Breazeale Nuclear Reactor pool. The goal was to experimentally verify that single-frequency emitted sine waves could be resolved in the reactor pool with background noise produced from mechanical pumps. This required the placement of two hydrophones, an underwater sound source, and a single axis accelerometer within the reactor pool. Both hydrophones and the sound source were submerged at the bottom of the pool, while the accelerometer was mounted atop an instrumentation dolly directly in contact with the water. A dynamic signal analyzer, oscilloscope, amplifier, and digital recorder were used as data acquisition tools during those measurements. The data taken by the digital recorder, in conjunction with MATLAB $^{\circledR}$ computational software, were used to produce the figures and graphics included in this summary.

\section{$3.1 \quad$ November 12, 2014}

Three measurement sets were taken under different operating conditions: two without the coolant pump running and one with the coolant pump running. The first two sets included the emission of three single-frequency sine waves $(1234 \mathrm{~Hz}, 1579 \mathrm{~Hz}, 2345 \mathrm{~Hz}$ ), one without the coolant pump running and one with the coolant pump running. The third set acquired six different reverberation measurements $(1.6 \mathrm{kHz}, 1.8 \mathrm{kHz}, 2 \mathrm{kHz}, 2.2 \mathrm{kHz}, 2.4$ $\mathrm{kHz}$, broadband white-noise) using the steady-state cut-off method without the coolant pump running [15]. Figure 3.1 shows the locations of the transducers for the experiments that follow. Figure 3.2 is a 3 -D rendering of the transducer positions. Throughout this thesis, the underwater sound source is labeled USS, and the two hydrophones and accelerometer are labeled H-007, H-008, and Accel, respectively. Both hydrophones were HTI-96 models and the accelerometer was a PCB-321A02. Their sensitivities and calibrations are summarized in Appendix A. 


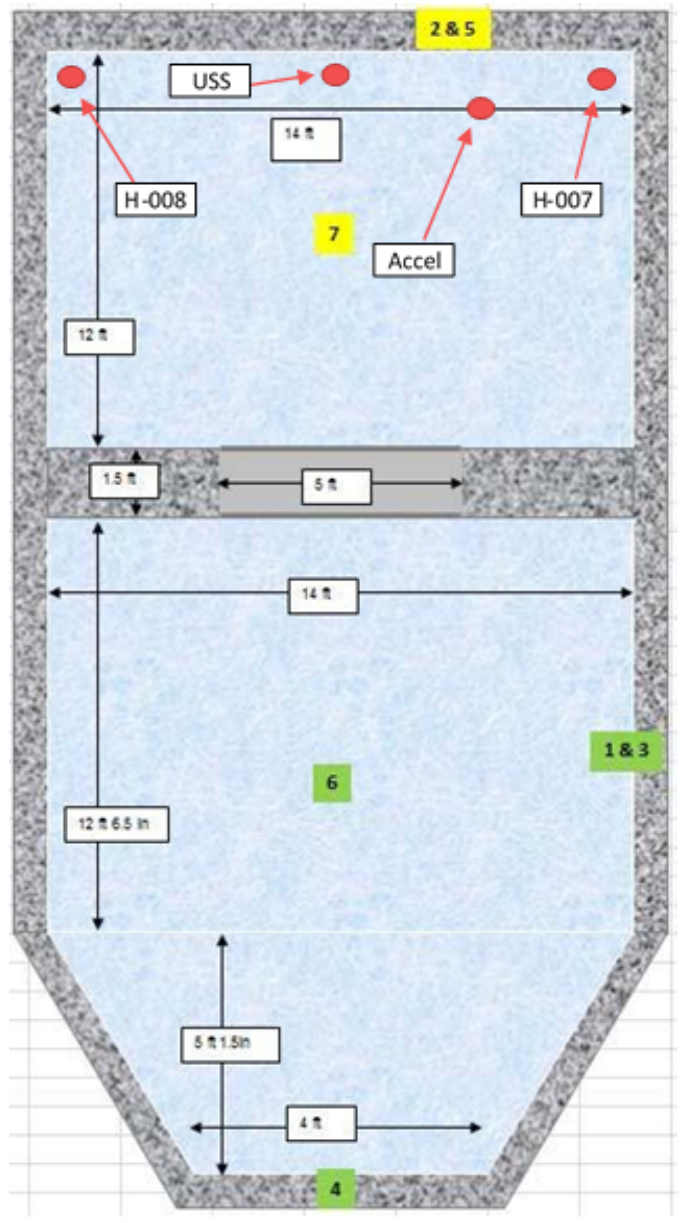

Figure 3.1: Transducer placement in the Breazeale reactor pool during the November 12, 2014 measurements. The red ovals represent the respective transducer. All except the accelerometer were placed at the bottom of the pool. The accelerometer was placed on an instrumentation dolly that was in direct contact with the water (see Fig. 3.9). A 3dimensional rendering of the source and sensor locations is provided in Fig. 3.2. 


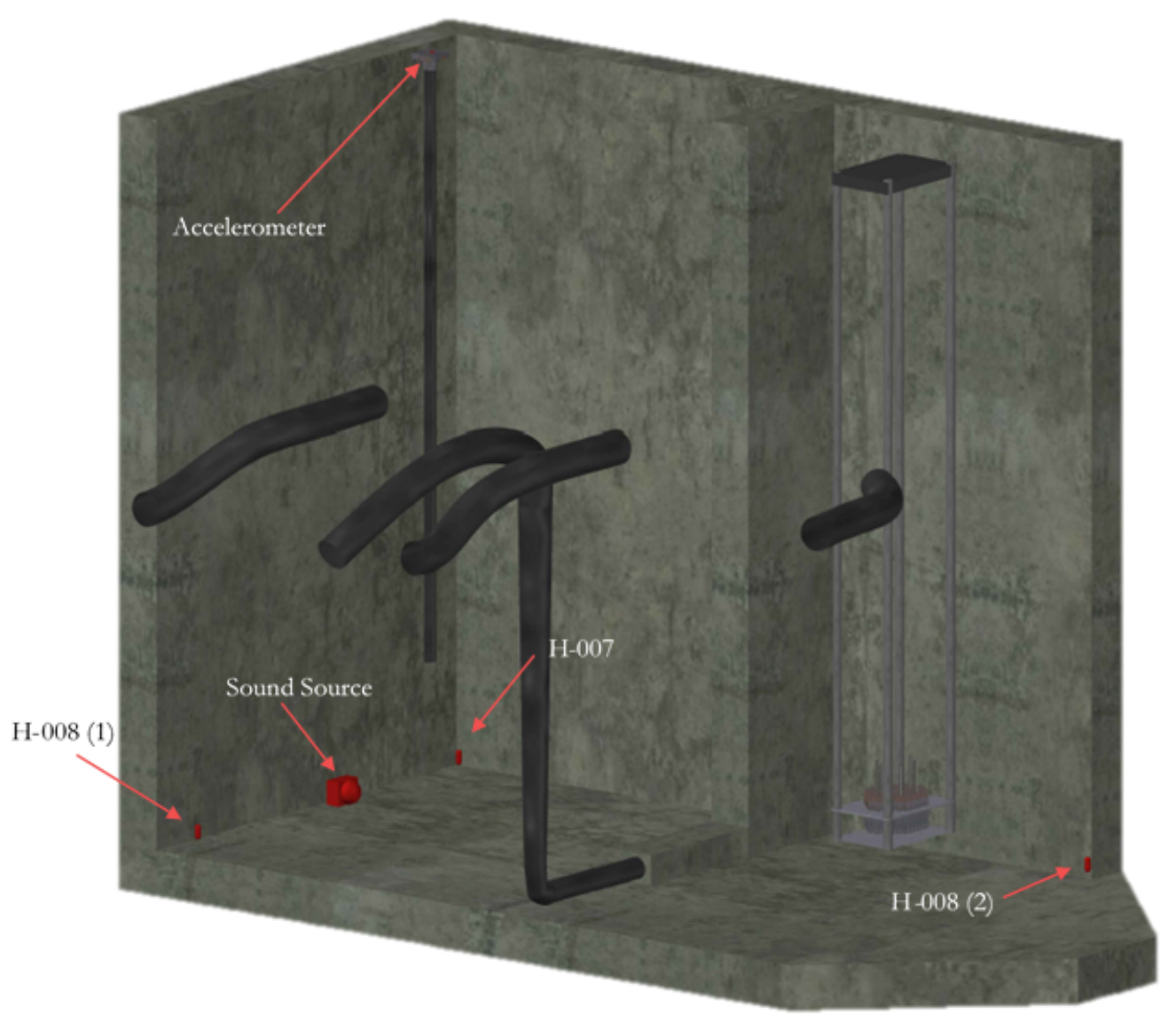

Figure 3.2: Transducer placement in the Breazeale reactor pool during the November 12, 2014 and December 04, 2014 measurements. The left-hand side of the figure represents the north bay and the right-hand side the south bay. The red objects represent the respective transducer. H-008(1) represents the hydrophone placement on November $12^{\text {th }}$, and H-008(2) represents the hydrophone after it was move for the December $4^{\text {th }}$ measurements. For more information on the reactor pool visualization refer to the Fig. 3.10 and Appendix B. 


\subsubsection{Coolant Pump Deactivated}

The reactor coolant pump is just one of three major pumps used in the reactor pool. It is used to circulate water throughout the pool to cool the reactor core. The coolant pump, along with the Nitrogen-16 diffuser pump, are the main sources of noise that interfere with the sensors' ability to achieve high signal-to-noise. As a result, measurements with and without the coolant pump were taken. Later, measurements with the ${ }^{16} \mathrm{~N}$ diffuser pump were also taken. The third pump, the filtration pump, does not introduce a significant amount of noise and will therefore be included as part of the total noise when the ${ }^{16} \mathrm{~N}$ diffuser pump is activated. To start, the coolant pump was deactivated, as well as all other significant noise sources that were not needed for "normal operation" of the Breazeale reactor.

The underwater sound source was a Lubell-LT9162T. It was driven by a 2.7 Volt (rms) voltage source with three simultaneous frequencies produced by an HP-8904A multifunction synthesizer at $1234 \mathrm{~Hz}, 1579 \mathrm{~Hz}$, and $2345 \mathrm{~Hz}$. This resulted in measured pressure levels between $100 \mathrm{~Pa}-500 \mathrm{~Pa}$, or underwater sound pressure levels (SPL) of $160 \mathrm{~dB}$ - $173 \mathrm{~dB}$ re $1 \mu \mathrm{Pa}$.

Figure 3.3 shows the received time series of all three transducers in the reactor pool. The top two plots are the hydrophones H-008 (upper) and H-007 (middle) and the bottom plot is the accelerometer. Figure 3.4 is a plot of the power spectra of Fig. 3.3 above to show the frequency content and amplitude of the received signals. The power spectra are represented in terms of root-mean-square pressure amplitudes. This was done by multiplying the double-sided power spectrum by the frequency separation between frequency bins. This product was then square-rooted and plotted to produce a rough estimate of the amplitude of a sine wave at a given frequency. This method, of course, does not account for signal processing phenomena such as leakage or attenuation due to windowing.

Figures 3.5 - 3.7 show zoomed-in views of each individual transducer's power spectrum. Since this was the first measurement, the accelerometer performance was surprisingly good, considering the arbitrary choice of mounting location (see Fig. 3.9). The spectrogram in Fig. 3.8 shows three very clear tonal signals, signifying that hydrophone-008 had a high signal-to-noise ratio. 
Time Series of Reactor Background Noise with Coolant Pump Off

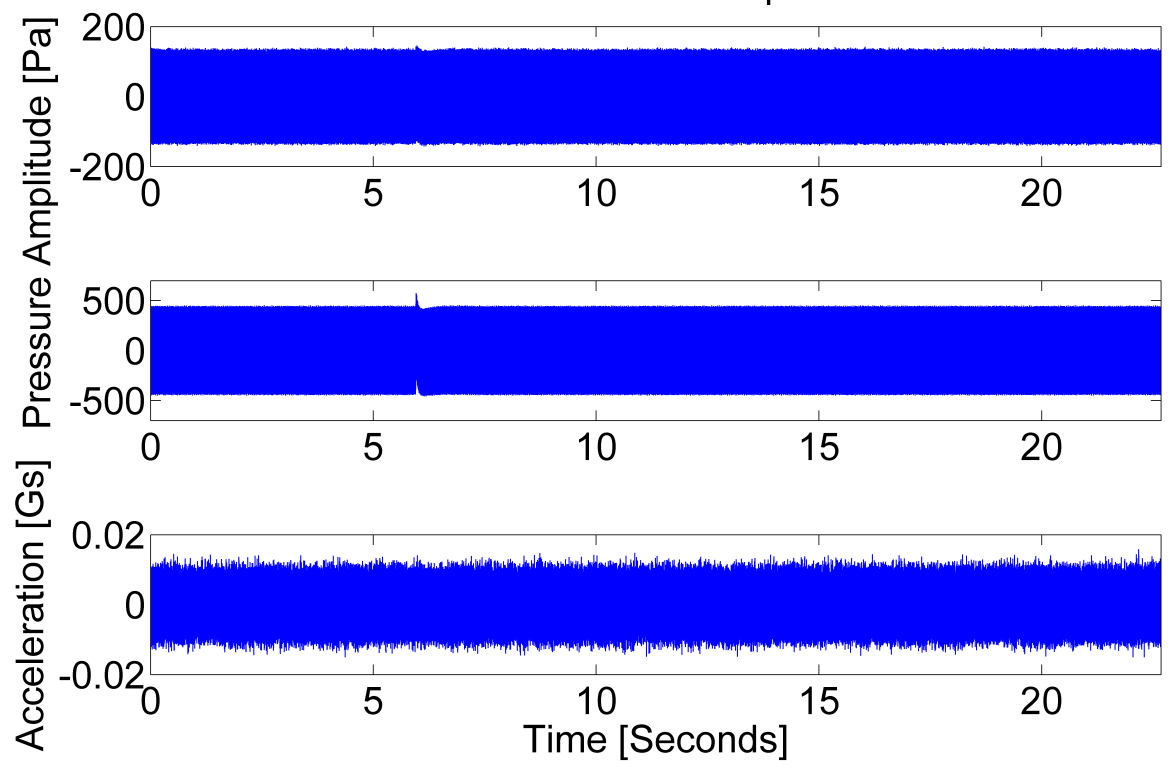

Figure 3.3: Time series of tri-tone frequencies while the coolant pump was deactivated. From top to bottom: hydrophone-008, hydrophone-007, and the accelerometer. The long time series was recorded to ensure ample signal-to-noise ratio.

Power Spectra of Tri-Tone [1234Hz, $1579 \mathrm{~Hz}, 2345 \mathrm{~Hz}]$ in Reactor with Coolant Pump Off

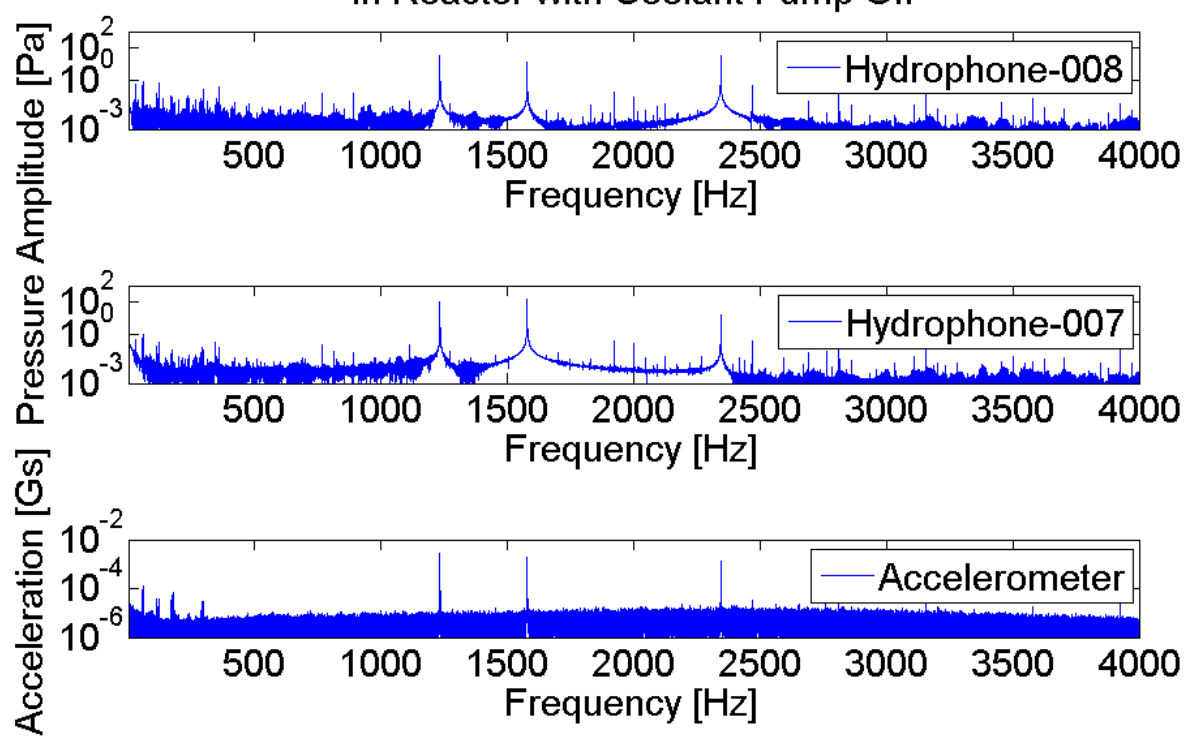

Figure 3.4: Power spectra from the time records of Fig. 3.3 for all three transducers while the tri-tone was emitted during the coolant pump deactivation. Each tonal frequency was more than two orders of magnitude above the noise for all three transducers. 
Power Spectra of Tri-Tone [1234Hz, 1579Hz, 2345Hz]

in Reactor with Coolant Pump Off

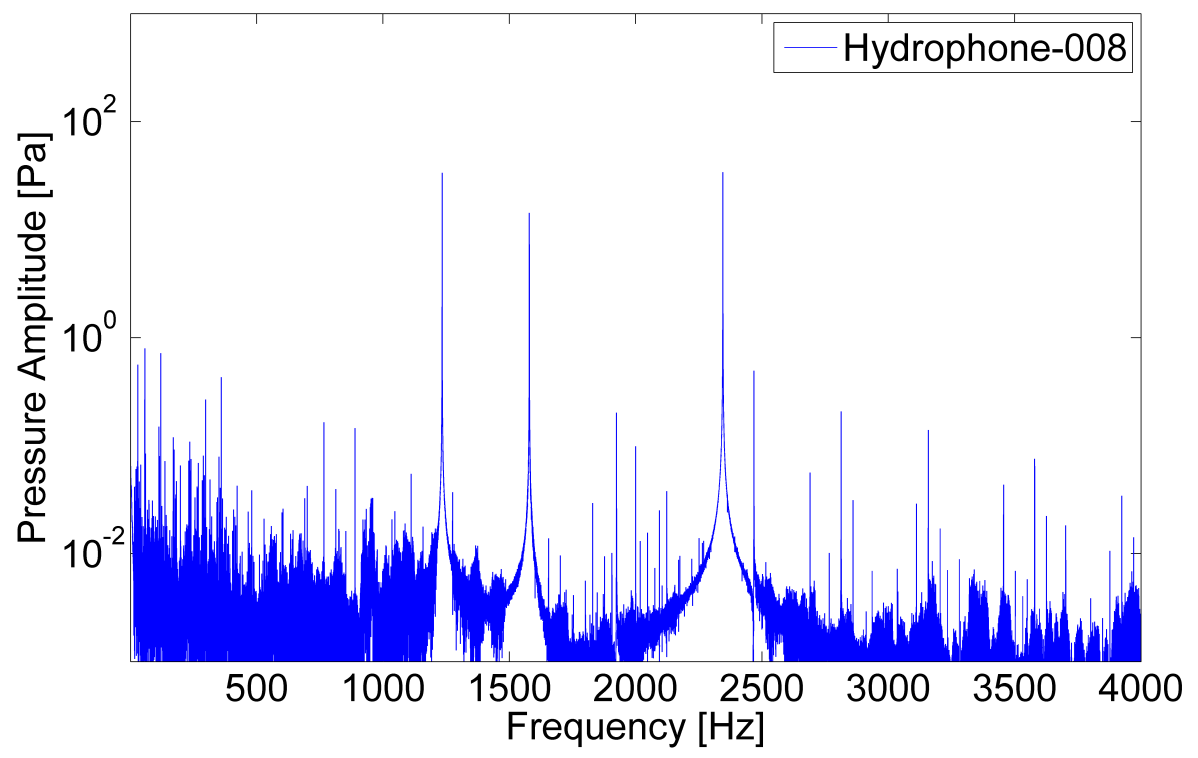

Figure 3.5: Power spectrum of a 23 second time window measurement of hydrophone-008 during the pump off measurement based on the time history of Fig. 3.3.

Power Spectra of Tri-Tone [1234Hz, 1579Hz, 2345Hz] in Reactor with Coolant Pump Off

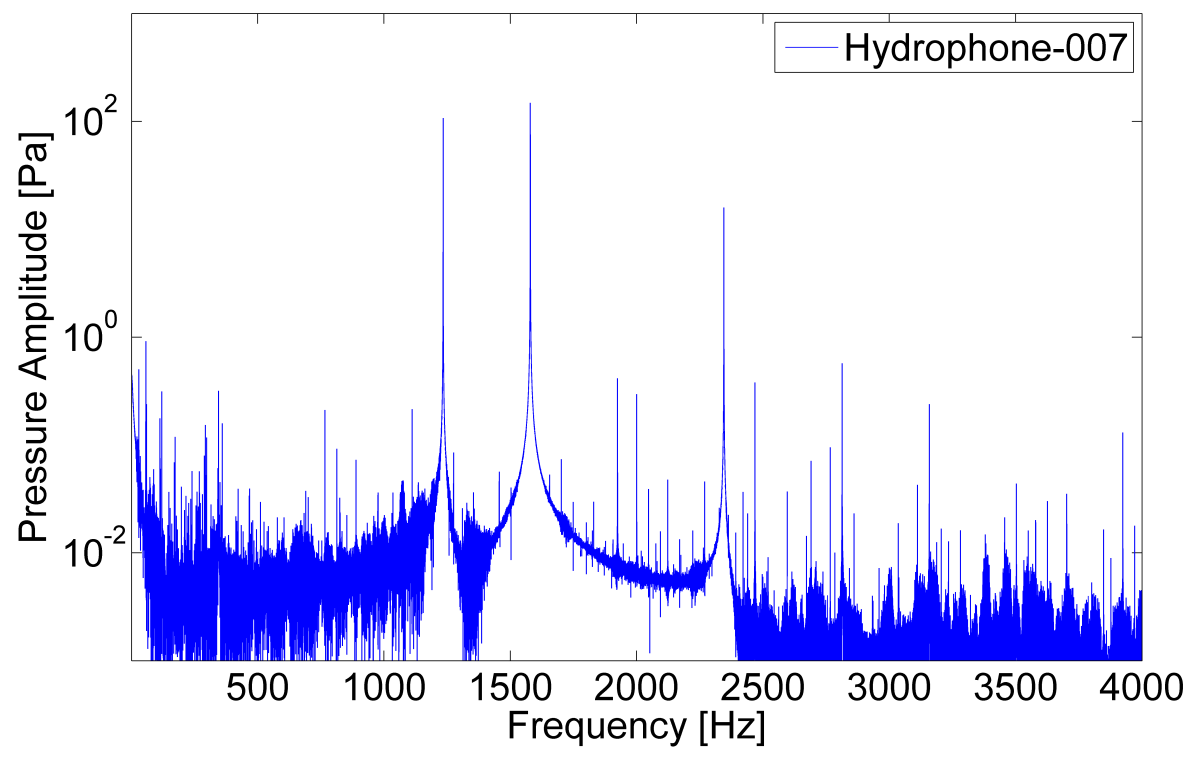

Figure 3.6: Power spectrum of a 23 second time window measurement of hydrophone-007 during the pump deactivation measurement based on the time history of Fig. 3.3. 


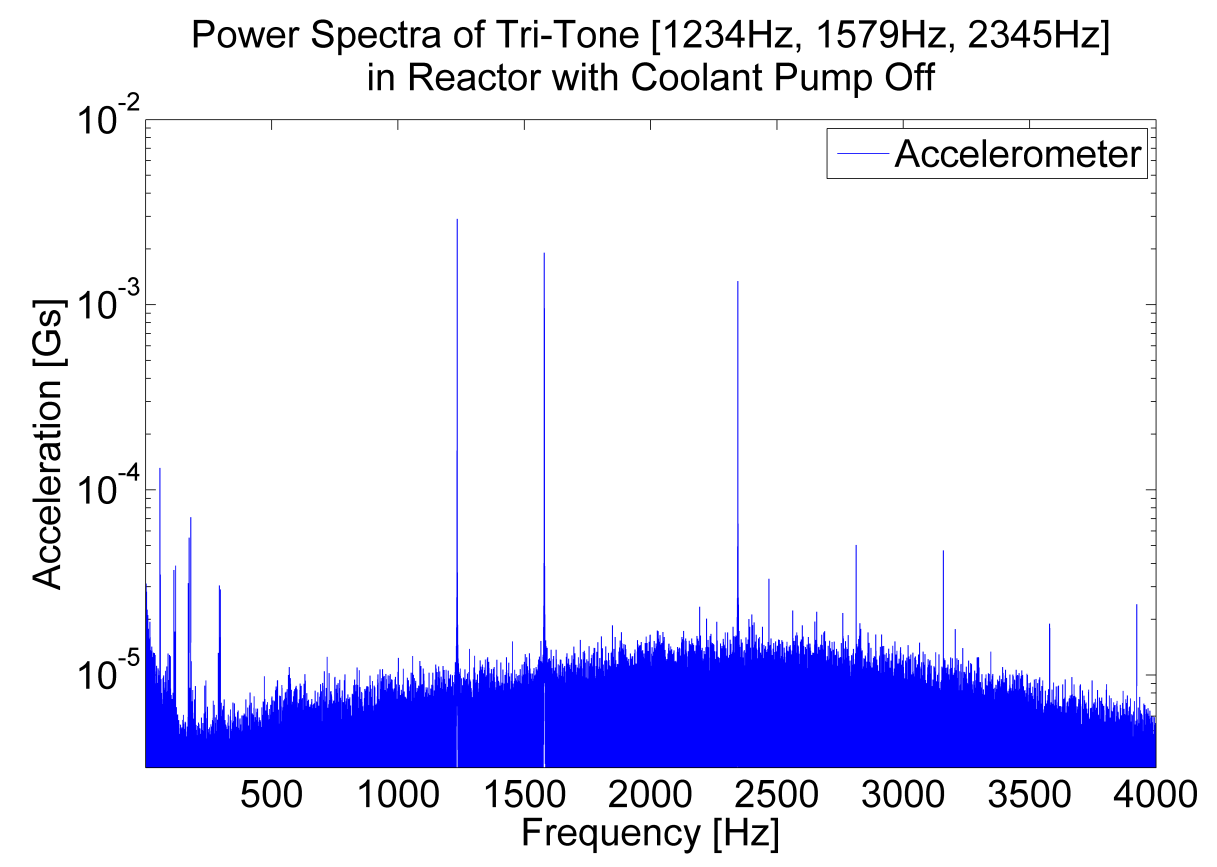

Figure 3.7: Power spectrum of a 23 second time window measurement of the accelerometer during the pump deactivation measurement based on the time history of Fig. 3.3.

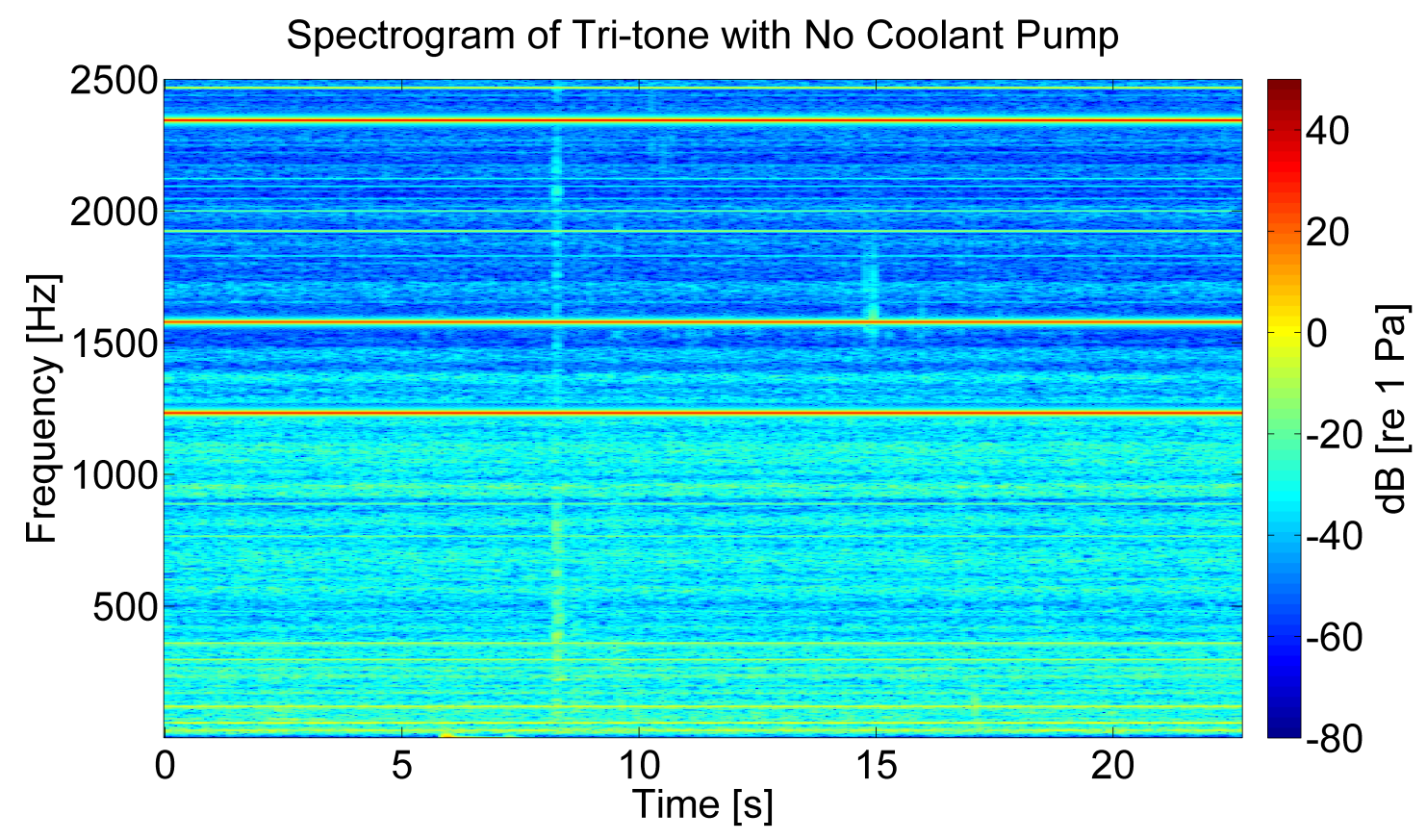

Figure 3.8: Spectrogram of a 23 second time window measurement of hydrophone-008 with the coolant pump deactivated. A 0.37 second Hann window $(2.7 \mathrm{~Hz}$ frequency bin) and $85 \%$ overlap between time records was used to enhance smoothing. No averaging between power spectra was done. 


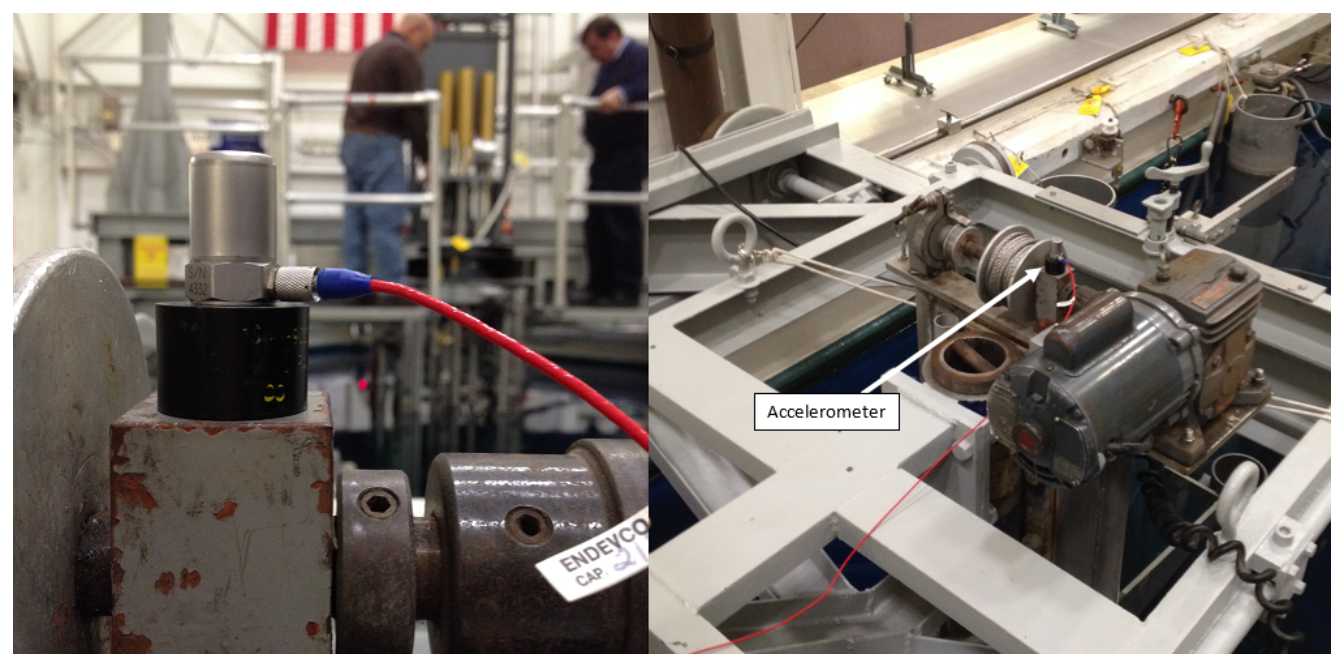

Figure 3.9: Accelerometer placement on the instrumentation dolly in the Breazeale reactor pool. 


\subsubsection{Coolant Pump Activated}

Figure 3.10 is a model of the reactor pool indicating the primary pump noise locations. The coolant pump is located a few feet below the surface of the pool and is labeled "coolant pump outlet" in the figure. The coolant pump represents the primary noise source for the following measurements.

Figure 3.11 is a plot of the 226 second time series recording for all three transducers after the activation of the coolant pump. Hydrophone-007 experiences some interference over time and was due to the interaction between the hydrophone's cable and a nearby bobbing iron pole in the water. The interference modulated the amplitude of the time series, as is evident in Fig. 3.11, but the frequency domain was only minimally affected. Notice that the effect present in the time series only minimally affects the spectrogram data. This verifies the theory of the broadband interference, and therefore, allows further analysis of the data despite the interference. A Hann window 0.186 second long $(5.38 \mathrm{~Hz}$ bin) and 50\% overlap between time records was used to produce the frequency vs. time spectrogram of H-007 in Fig. 3.13. The chosen length of the Hann window and percentage of overlap were chosen such that frequencies in the $2 \mathrm{kHz}$ range could cycle at least $5-10$ times before new data points were introduced.

The three tones $(1234 \mathrm{~Hz}, 1579 \mathrm{~Hz}, 2345 \mathrm{~Hz})$ are resolved clearly in the spectrum, despite the obvious rise in background noise caused by the coolant pump. Figure 3.12 shows the power spectrum for each transducer. The activation of the coolant pump does not obscure the tonal frequencies in the spectrum. Figures 3.14-3.16 show the individual power spectrum for each transducer. The underwater sound source operated under conditions identical to that of the pump deactivation, and produced high SNR despite the increased background noise level. In the next section, the frequency content of the added pump noise will be explored, and in later sections a few methods for filtering and isolating the signals will be presented. 


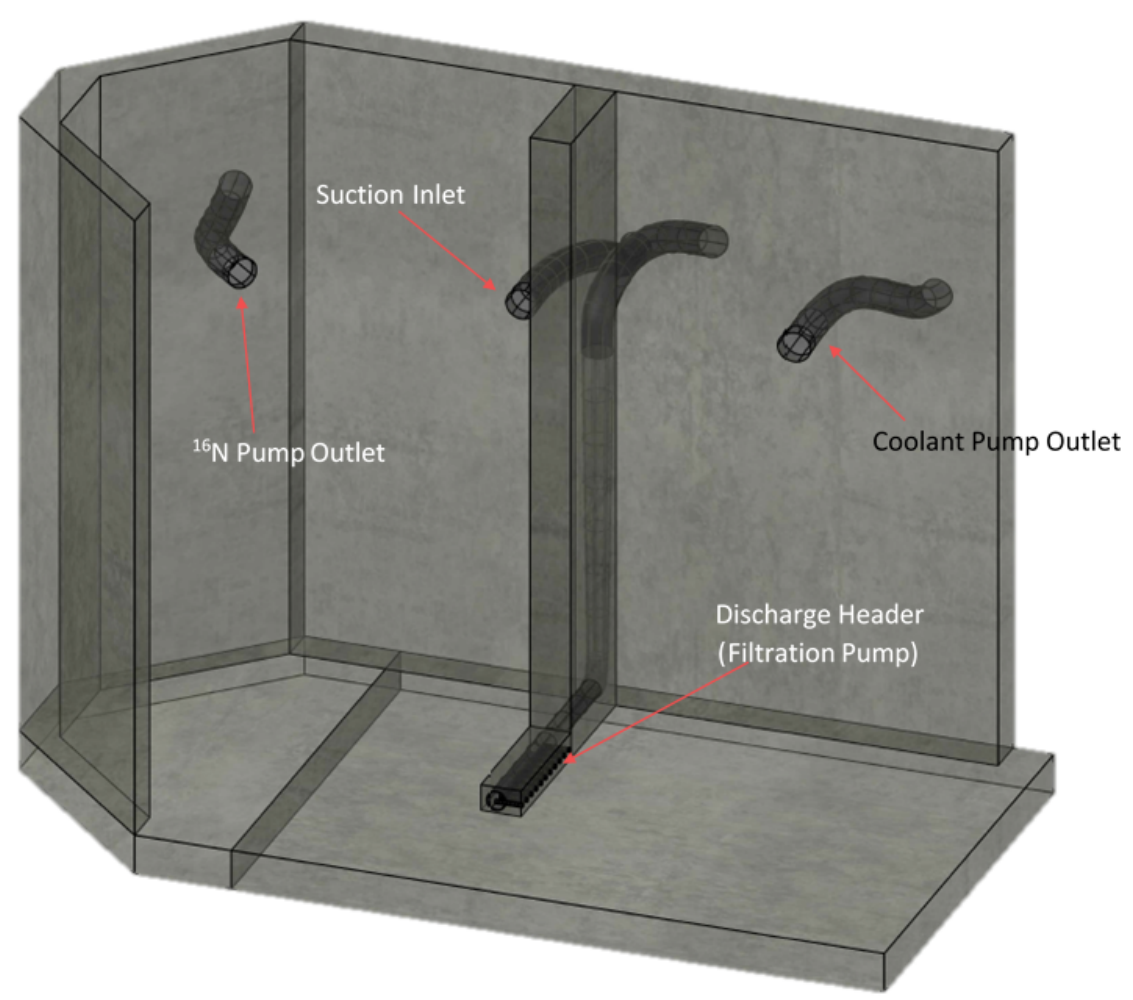

Figure 3.10: AutoCAD ${ }^{\circledR}$ rendering of the primary noise sources due to pump fluid flow. The left side of the figure represents the south bay where the reactor core is typically located. The ${ }^{16} \mathrm{~N}$ pump discharges toward the top of the core assembly, while the filtration pump discharges from the bottom of the pool. The coolant pump discharges from the north bay where the transducers were located. 


\section{Time Series of Reactor Background Noise}

With Coolant Pump On

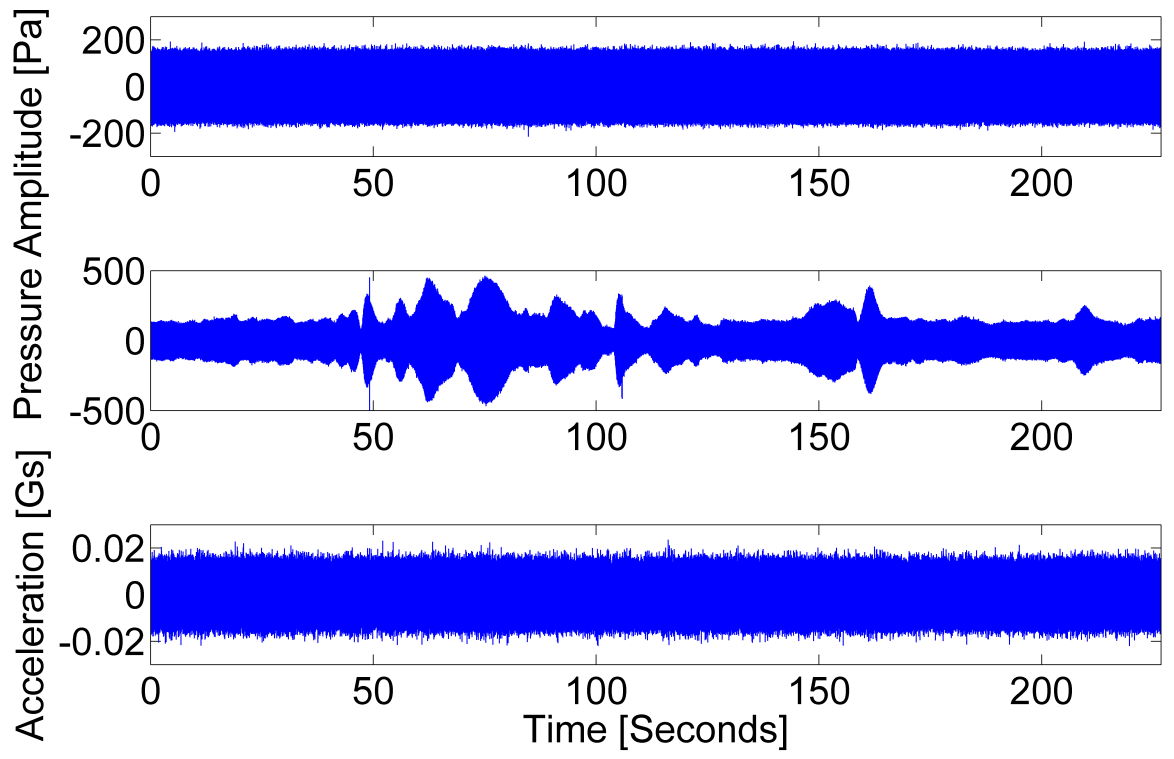

Figure 3.11: Time series of tri-tone frequencies after coolant pump activation. The axes are identical to those in Fig. 3.3, from top to bottom: hydrophone-008, hydrophone-007, and the accelerometer. Notice the fluctuations in H-007. This was due to interaction between the hydrophone's cable and a nearby randomly-bobbing pipe. Some interference caused the time series to fluctuate greatly, but the frequency domain is minimally impacted by the slow amplitude modulation, as shown Fig. 3.13. 


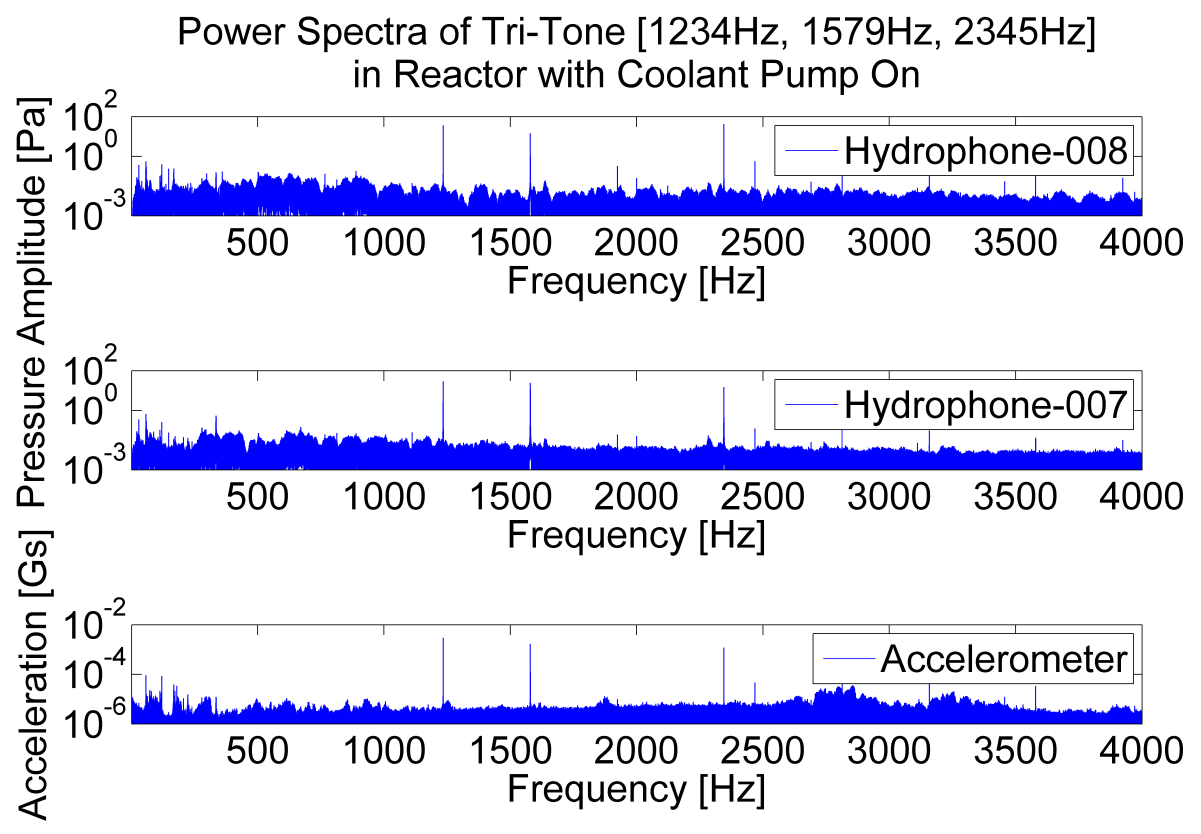

Figure 3.12: Power spectrum of each transducer after the pump activation. No debilitating effects are present when the coolant pump is running. Each transducer still shows a high signal-to-noise ratio.

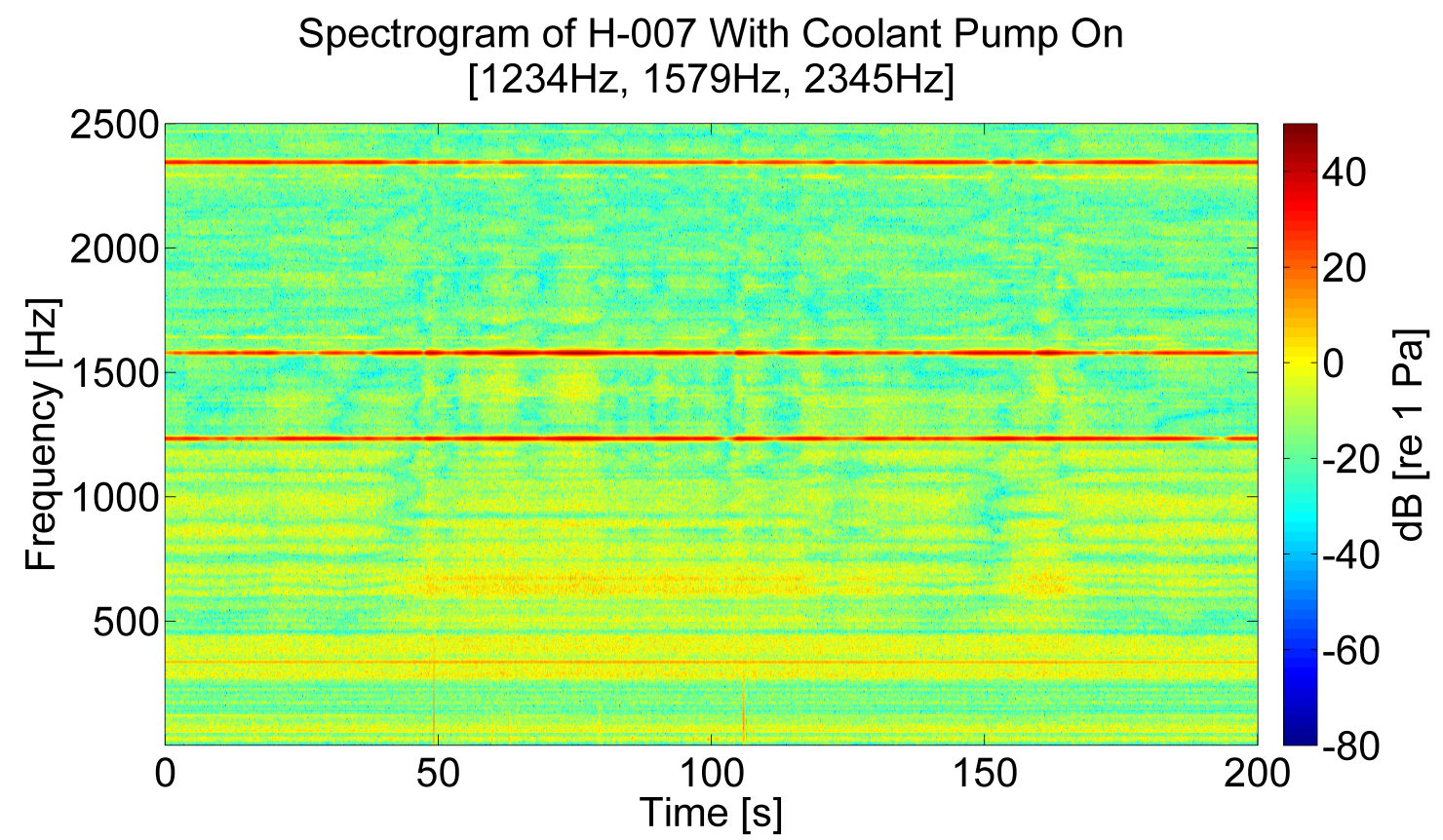

Figure 3.13: Spectrogram of a 200 second time window measurement of hydrophone-007 after the pump activation. A 0.186 second long $(5.38 \mathrm{~Hz}$ bin) Hann window was implemented without averaging and $50 \%$ overlap between time records was used to enhance smoothing. 


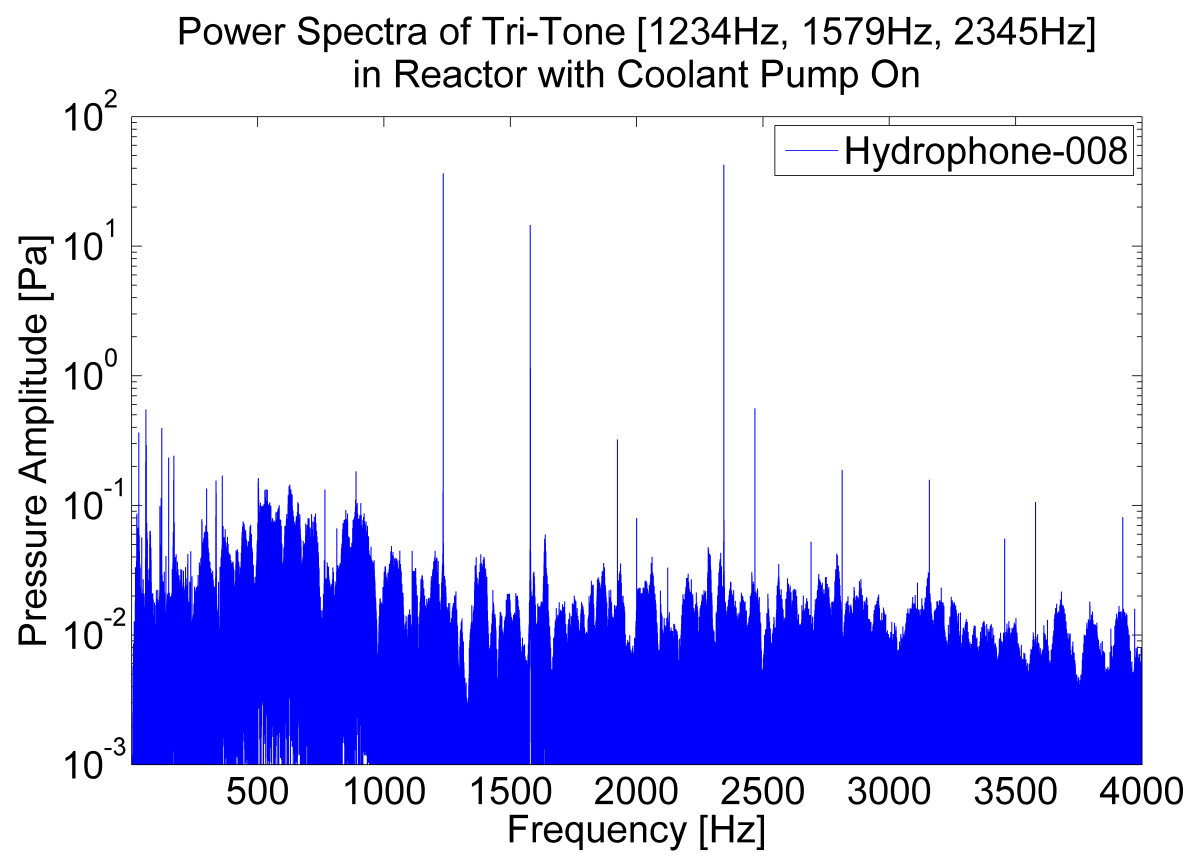

Figure 3.14: Power spectrum of a 226 second time window measurement of hydrophone-008 while the coolant pump was operating.

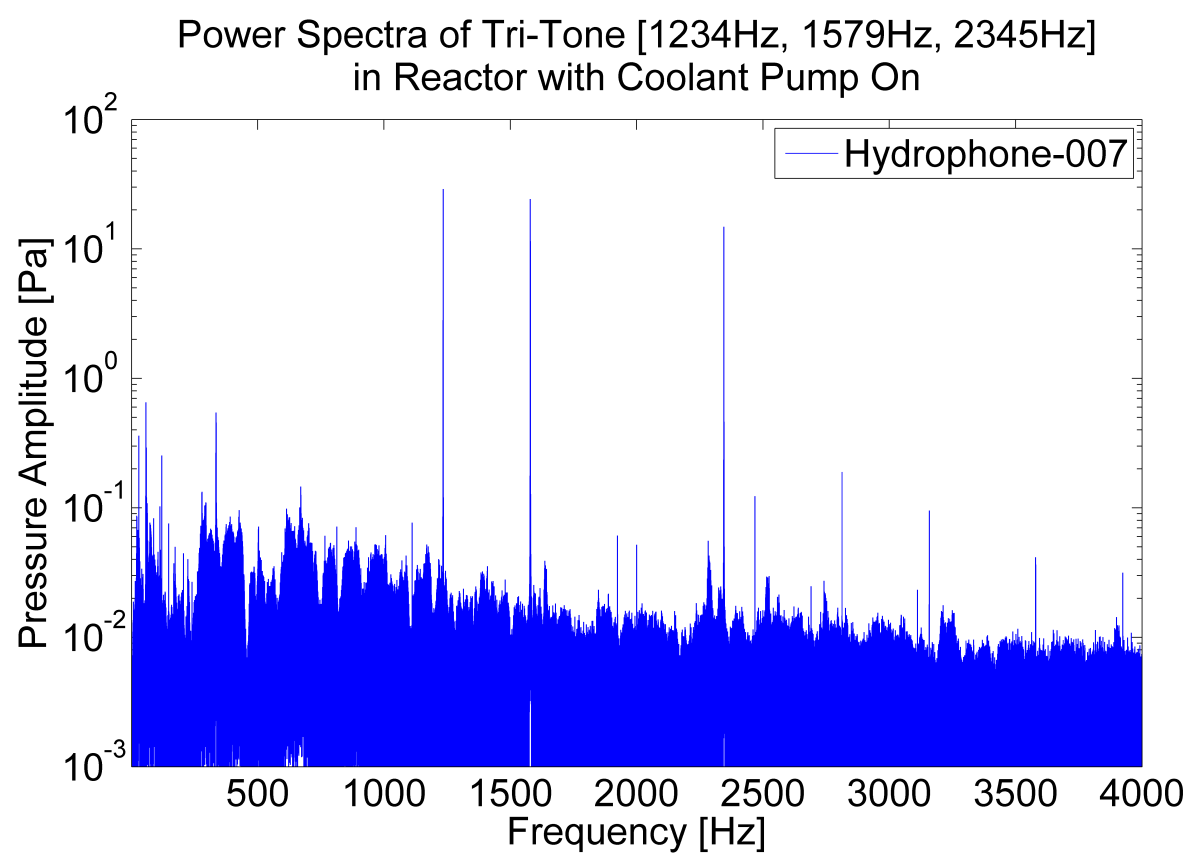

Figure 3.15: Power spectrum of a 226 second time window measurement of hydrophone-007 while the coolant pump was activated. 


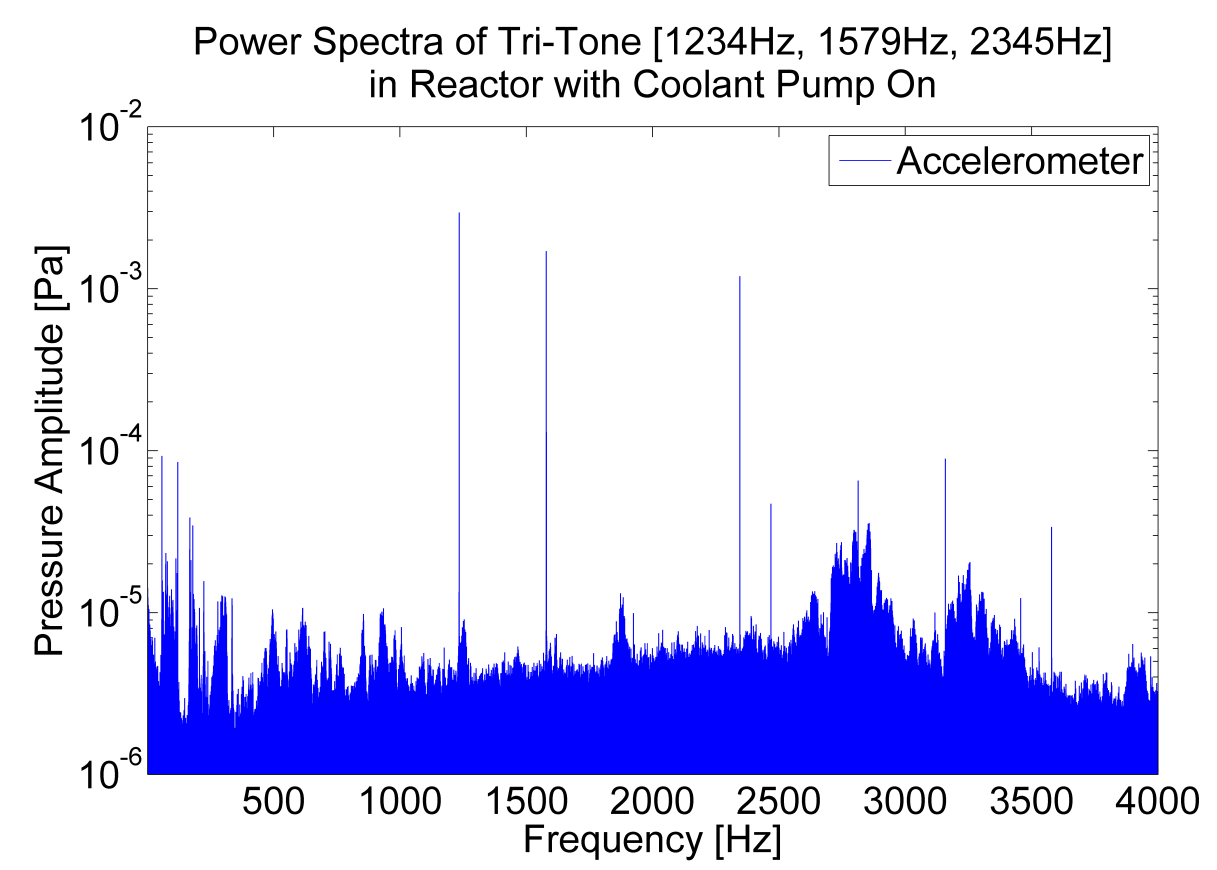

Figure 3.16: Power spectrum of a 226 second time window measurement of the accelerometer while the coolant pump was activated.

\subsubsection{Frequency Content of Pump Noise}

The operation of the coolant pump raised the noise floor without introduction of tonals that interfere with the sensor frequencies $(f>1.2 \mathrm{kHz})$. This is demonstrated by Figs. 3.17-3.22. The first three Figs. (3.17-3.19) contain the power spectra for each transducer with a frequency range from $0 \mathrm{~Hz}$ to $4 \mathrm{kHz}$. The second half of the plots (Figs. 3.20-3.22) are expanded views of the prominent noise (below $1 \mathrm{kHz}$ ).

The activation of the coolant pump raised minimal concern after comparing the responses from pump-on and pump-off measurements. The pump tripled the rms background noise power level. However, the original pump noise was low enough that the signal-tonoise ratio (SNR) remained adequate for detection of the tonals. Figures 3.19 and 3.22 are the accelerometer's response to the pump activation. The accelerometer measurement sensitivity was a big surprise in this measurement. It had a response comparable to the hydrophones, despite having been placed in an arbitrary location. While both hydrophones experienced a linear rise in the noise floor, the accelerometer appeared to experience more high-frequency noise. Figure 3.19 shows a rise in noise floor between $2.5 \mathrm{kHz}$ and $3.5 \mathrm{kHz}$. This could be attributed to a mechanical vibration mode of the instrumentation dolly upon activation of the coolant pump. Also, for a fixed displacement amplitude, $x$, the sensitivity of any accelerometer increases quadratically with increasing frequency by the relation: $a=\omega^{2} x$. Overall, the pump noise was dominant in the low frequency region for the hydrophones, far enough below the sensor frequencies to be filtered without attenuation of the sensor frequencies. Insertion of a high-pass filter, with a $-3 \mathrm{~dB}$ frequency in the 
range of $800 \mathrm{~Hz}$, between the sensor and the first stage of signal-conditioning electronics will improve the dynamic range of the data acquisition and analysis without significant impact on the tonals of interest. Filtering and isolation of these frequencies will be covered in later sections (see Sec. 3.3.2).

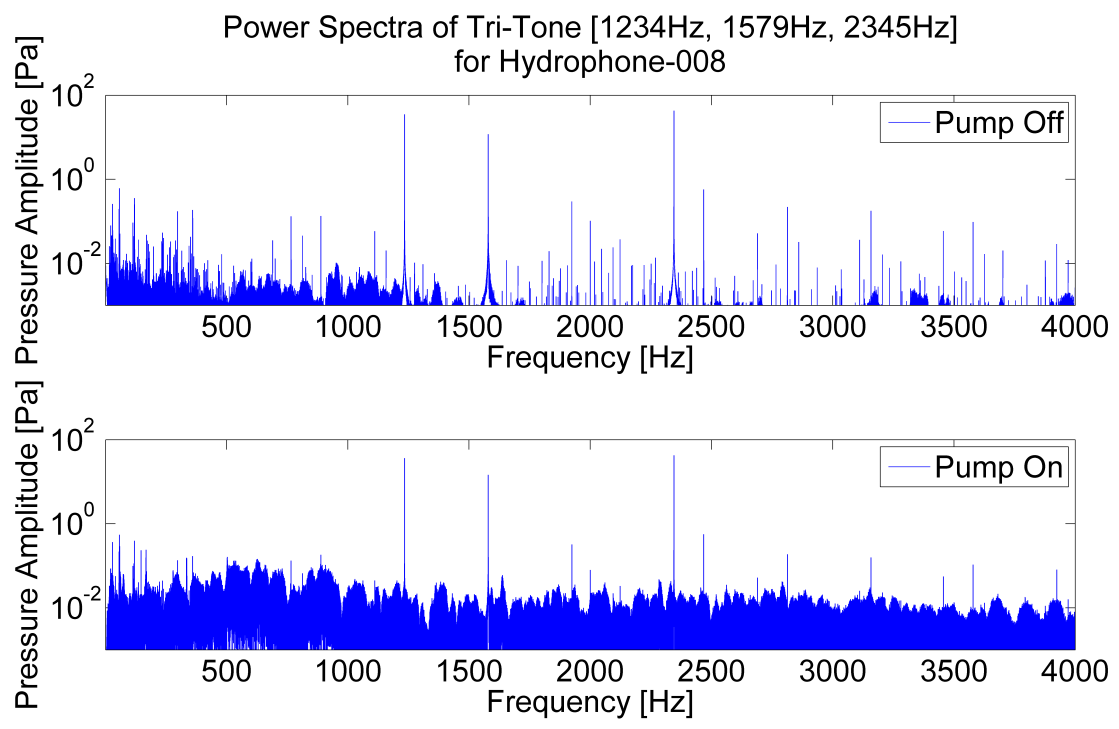

Figure 3.17: Power spectra for H-008 during pump-on and pump-off measurements. The noise floor is raised when the coolant pump is activated, but the tri-tone frequencies are seemingly unaffected and remain resolvable.

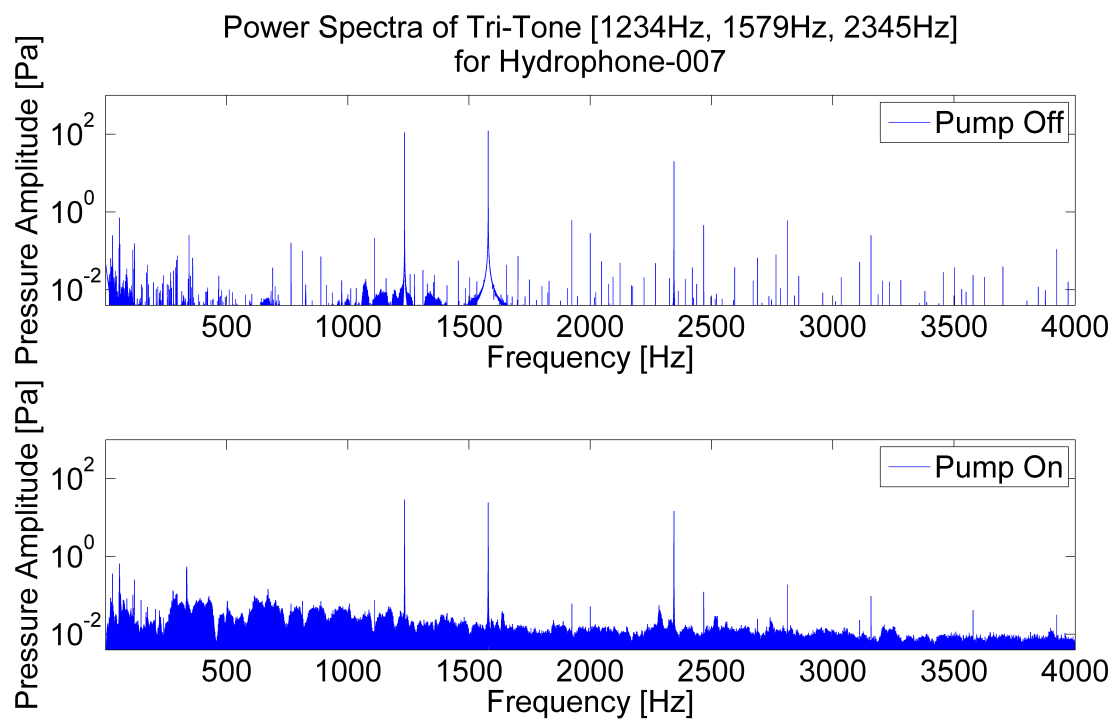

Figure 3.18: Power spectra for H-007 during pump-on and pump-off measurements. The noise floor is raised when the coolant pump is activated, but the tri-tone frequencies are unaffected and remain resolvable. 

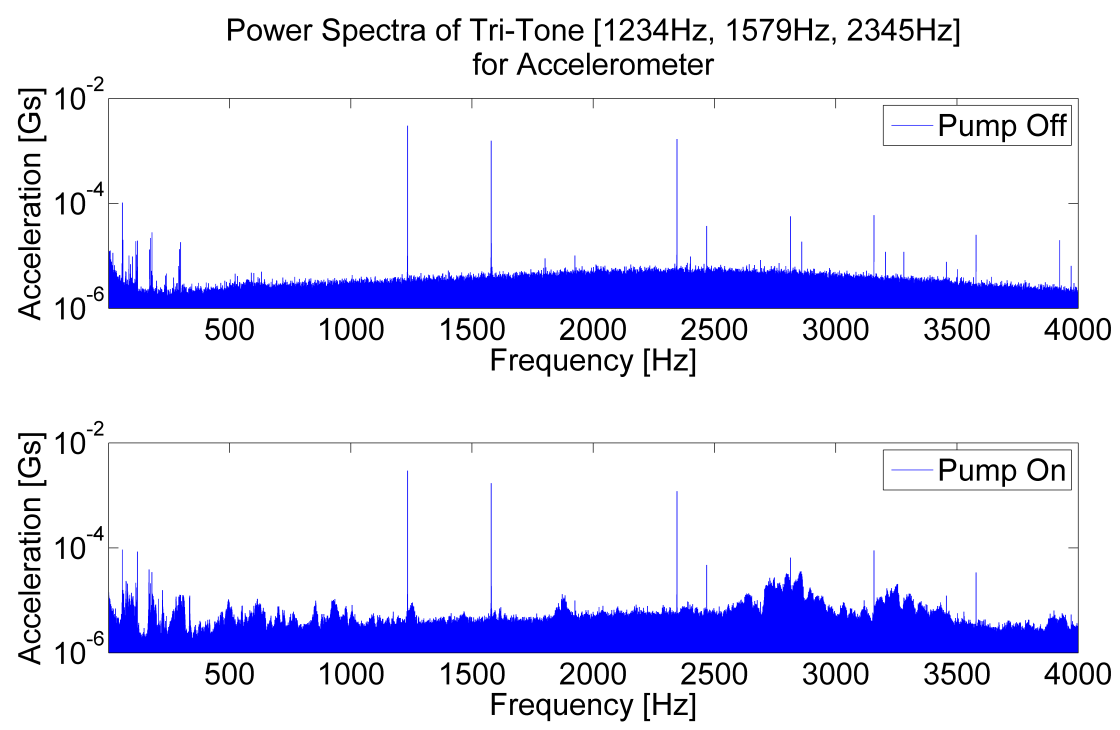

Figure 3.19: Power spectra for the accelerometer during pump-on and pump-off measurements. The noise floor is raised when the coolant pump is activated, but the tri-tone frequencies are well above the noise floor. It appears that a high frequency contribution to the noise exists between $2.5 \mathrm{kHz}$ and $3.5 \mathrm{kHz}$. This may be attributed to vibrational modes of the instrumentation dolly and to the nonlinear increase in accelerometer response with increasing frequency.

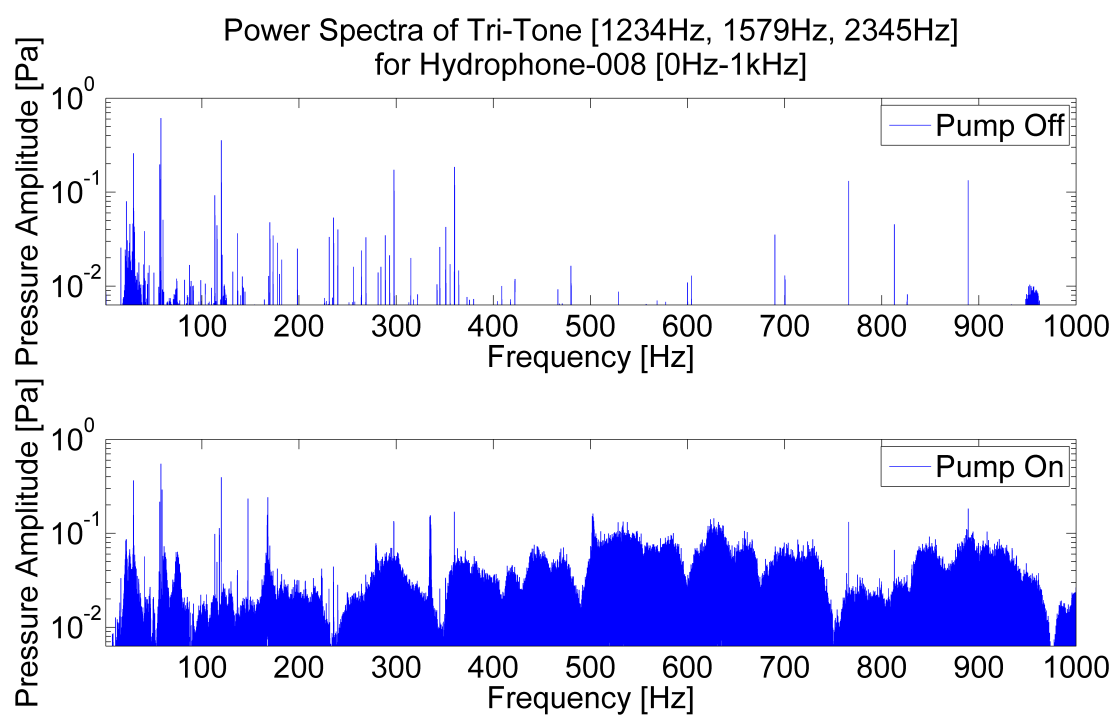

Figure 3.20: Low frequency power spectra for H-008 during pump-on and pump-off measurements. The broadband noise floor absorbs most tonals in the low frequency range. This low frequency noise can be high-pass filtered to prevent saturation of the signal-conditioning and data acquisition electronics for future measurements. 


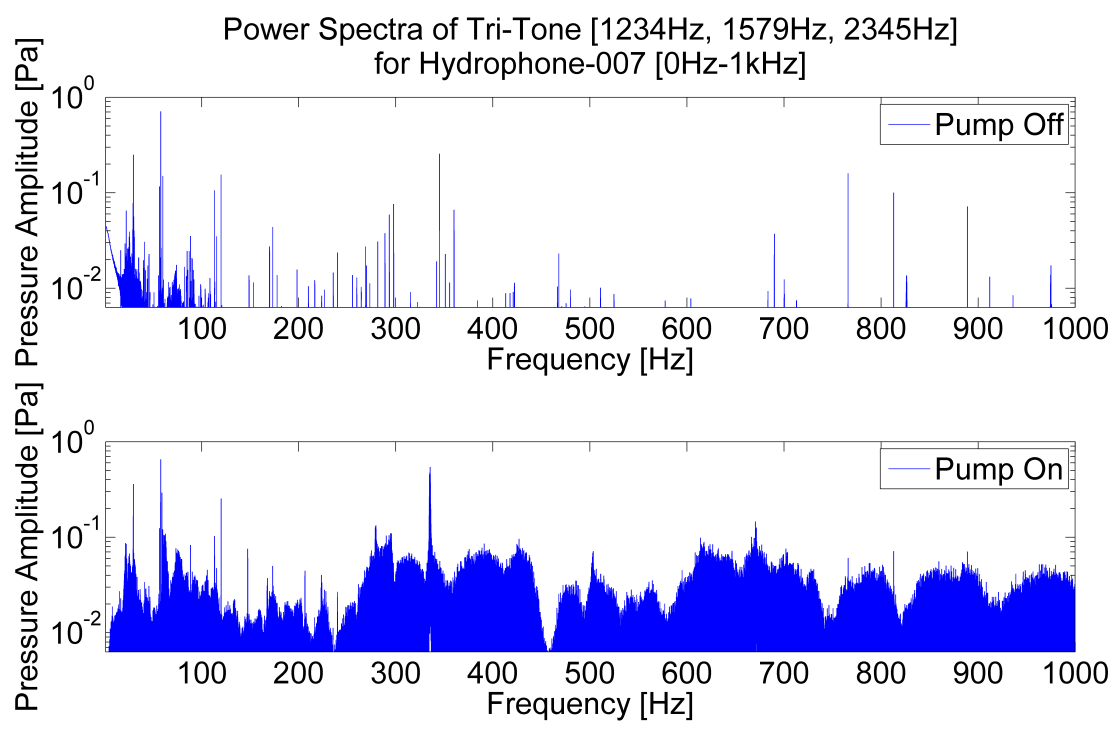

Figure 3.21: Low frequency power spectra for H-007 during pump-on and pump-off measurements. The broadband noise floor absorbs most tonals in the low frequency range. This low frequency noise can be high-pass filtered to prevent saturation of the signal-conditioning and data acquisition electronics for future measurements.
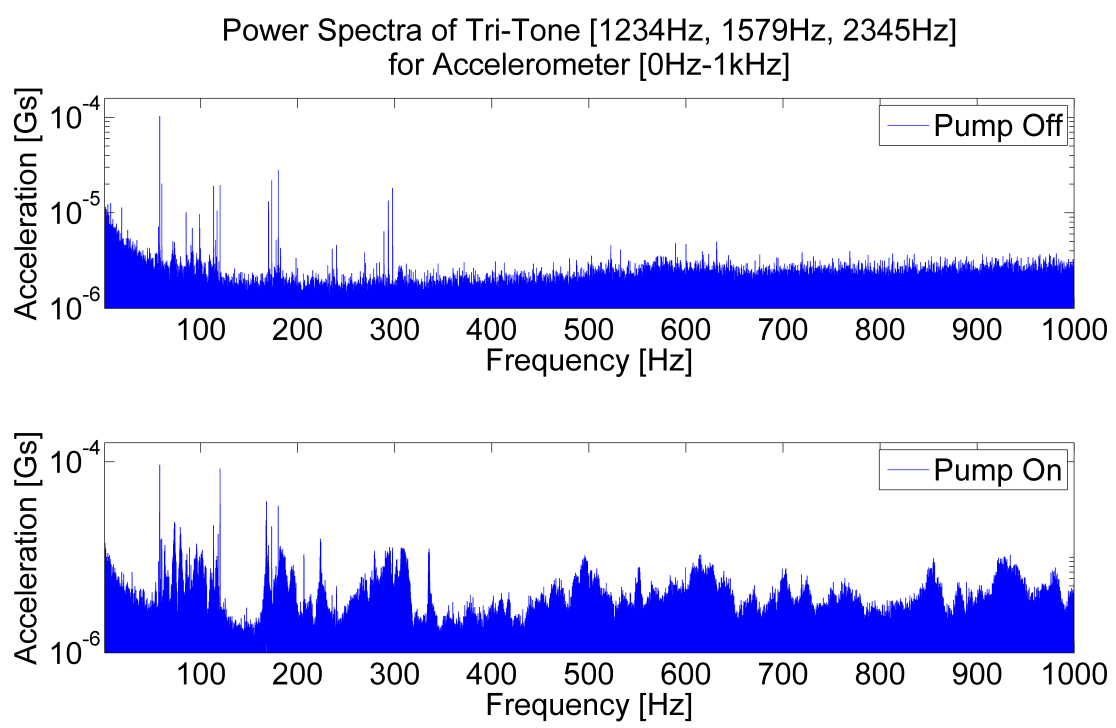

Figure 3.22: Low frequency power spectra for the accelerometer during pump-on and pumpoff measurements. The broadband noise floor absorbs most tonals in the low frequency range. This low frequency noise can be high-pass filtered to prevent saturation of the signal-conditioning and data acquisition electronics for future measurements.

\subsubsection{Reverberation Measurements}

The reverberation time measurement allows quantification of the losses in the pool and prediction of the steady-state amplitude of the sound produced by the acoustical power 
radiated from the fuel rod resonator under the assumption that the resultant sound field is diffuse. One way to characterize reverberant behavior is to use the steady-state cutoff method [15]. This involves sending a steady signal into an enclosure, such as the reactor pool, and then abruptly terminating the signal. Earlier in Sec. 2.3.2, the reverberation time was utilized to calculate the critical distance and the Schroeder cutoff frequency. Figure 3.23 shows six different periods of the steady-state cutoff, each produced by excitation at a different frequency. The first five records were excited by frequencies ranging from $1.6 \mathrm{kHz}$ to $2.4 \mathrm{kHz}$ in steps of $200 \mathrm{~Hz}$, and the sixth record consisted of broadband white noise. Figure 3.24 is the power spectra for the whole time series, and Fig. 3.25 shows a frequency spectrogram. The frequency peaks, despite being taken from short time bursts, are clear and easily detectable.

Table 3.1 shows all six steady-state $T_{60}$ values. $T_{60}$ is defined in Eq. 2.32 as the time it takes for a signal to decay $60 \mathrm{~dB}$ from its peak amplitude after the drive signal is terminated. Figure 3.26 is an example of the $2 \mathrm{kHz}$ time record that was used to calculate its corresponding value in Table 3.1. The $T_{60}$ values were extrapolated from the logarithmic amplitude of each decay period to determine an equivalent $60 \mathrm{~dB}$ drop. Most of the amplitudes did not drop $60 \mathrm{~dB}$ from their original amplitude because the noise floor was generally within $15 \mathrm{~dB}$ or $10 \mathrm{~dB}$ of the amplitude, so $15 \mathrm{~dB}$ or $10 \mathrm{~dB}$ drops were used to extrapolate the $60 \mathrm{~dB}$ decay time. These decay times can then be used to approximate equivalent sound absorption area of the walls of the reactor (see Eq. 2.28 and 2.32).

The reverberation times in Table 3.1 were due to the close proximity of the hydrophones and the source. It is possible that those "reverberation times" were actually the time it took the underwater sound source to decay after cutoff, rather than characterizing the diffuse sound field environment. To escape this artifact, hydrophone-008 was relocated across the length of the pool where it would be sufficiently far from any directly transmitted sound from the underwater sound source. In the next section, the results of the relocated hydrophone are more representative of the diffuse sound field in the reactor pool. 


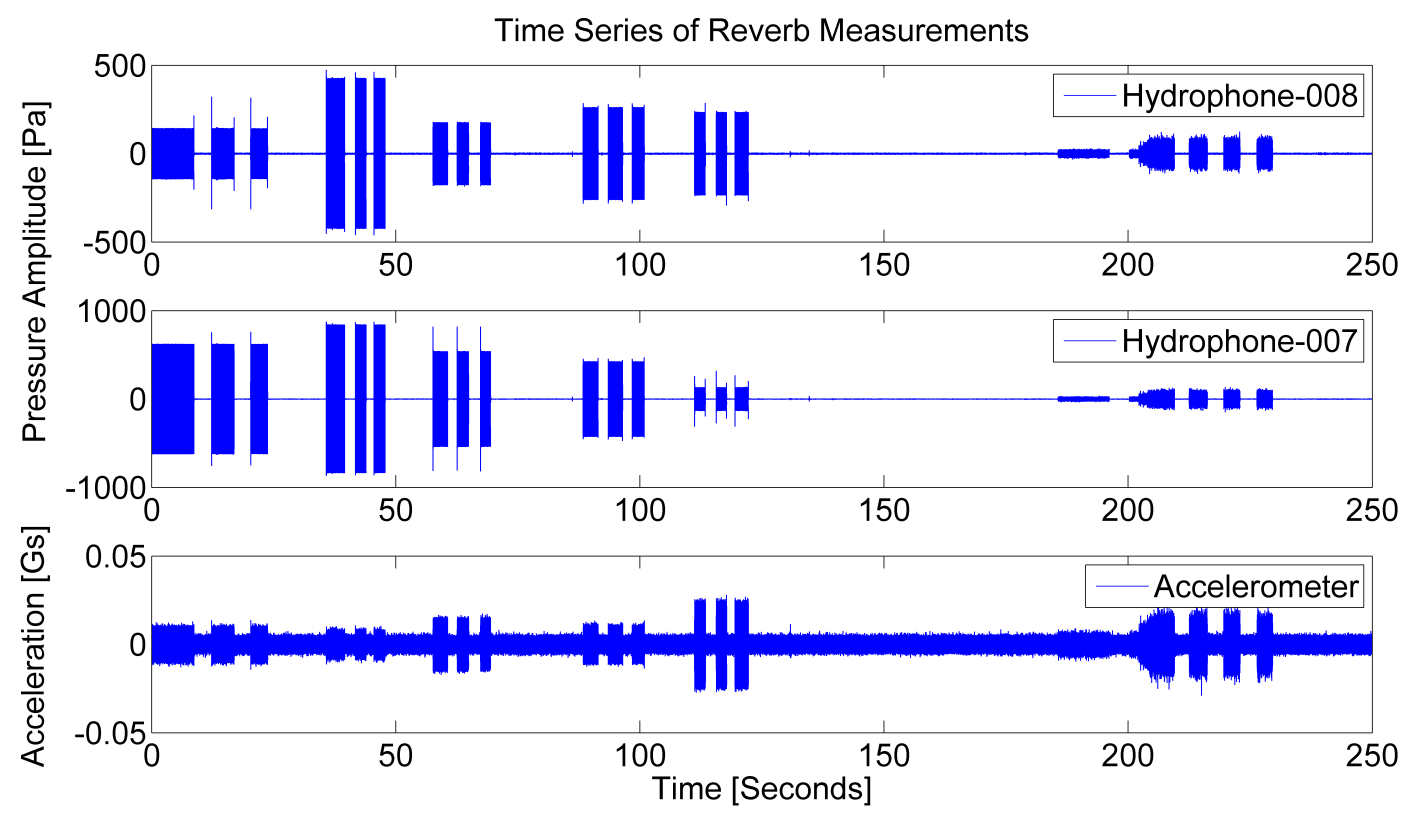

Figure 3.23: Time series of reverberation measurements without the coolant pump running. Each group of waveforms represents a separate steady-state excitation and its corresponding cutoff. The first five periods were frequencies ranging from $1.6 \mathrm{kHz}$ to $2.4 \mathrm{kHz}$ in steps of $200 \mathrm{~Hz}$, and the sixth period was excited by broadband white noise.
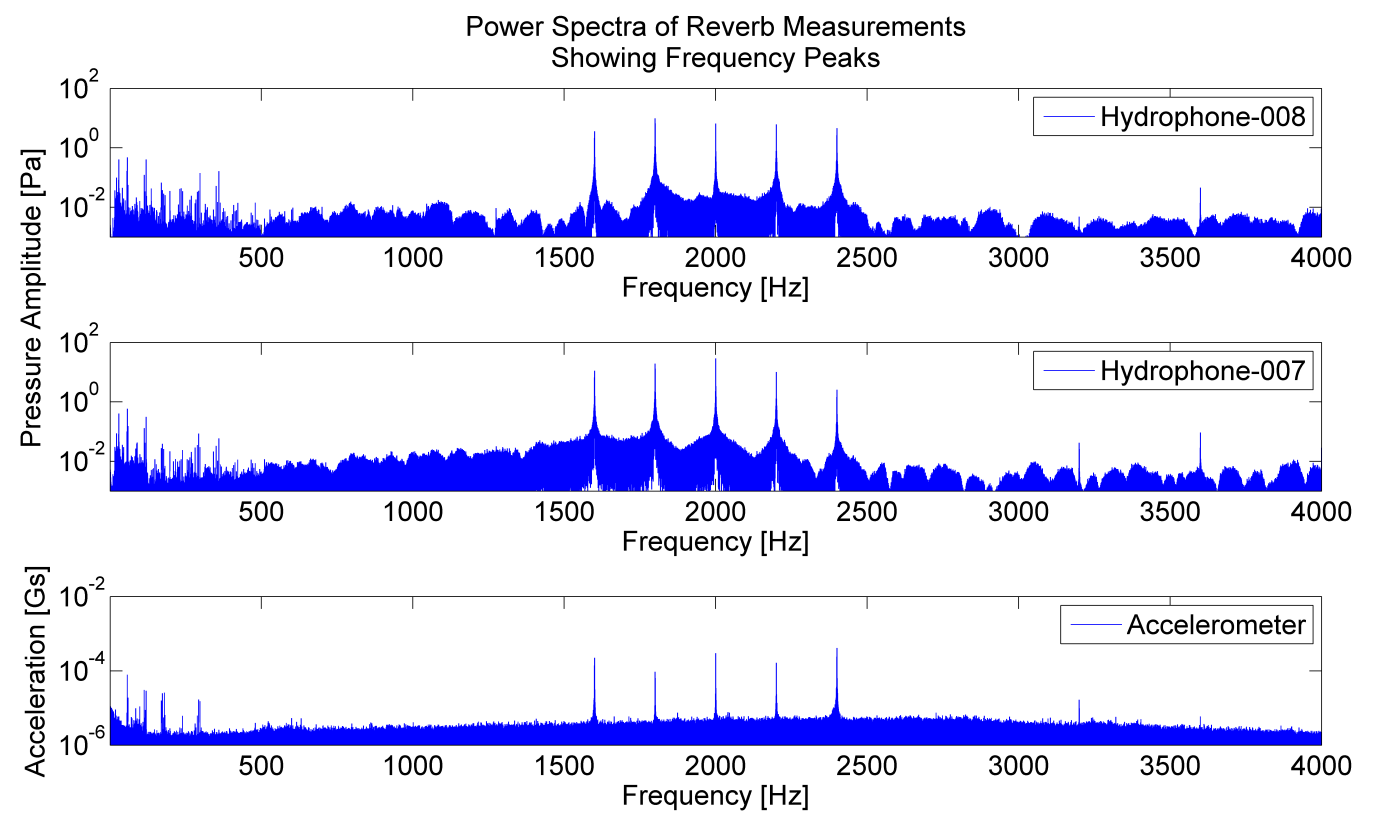

Figure 3.24: Power spectra showing the five frequency peaks from $1.6 \mathrm{kHz}-2.4 \mathrm{kHz}$ in steps of $200 \mathrm{~Hz}$ for all three transducers. 


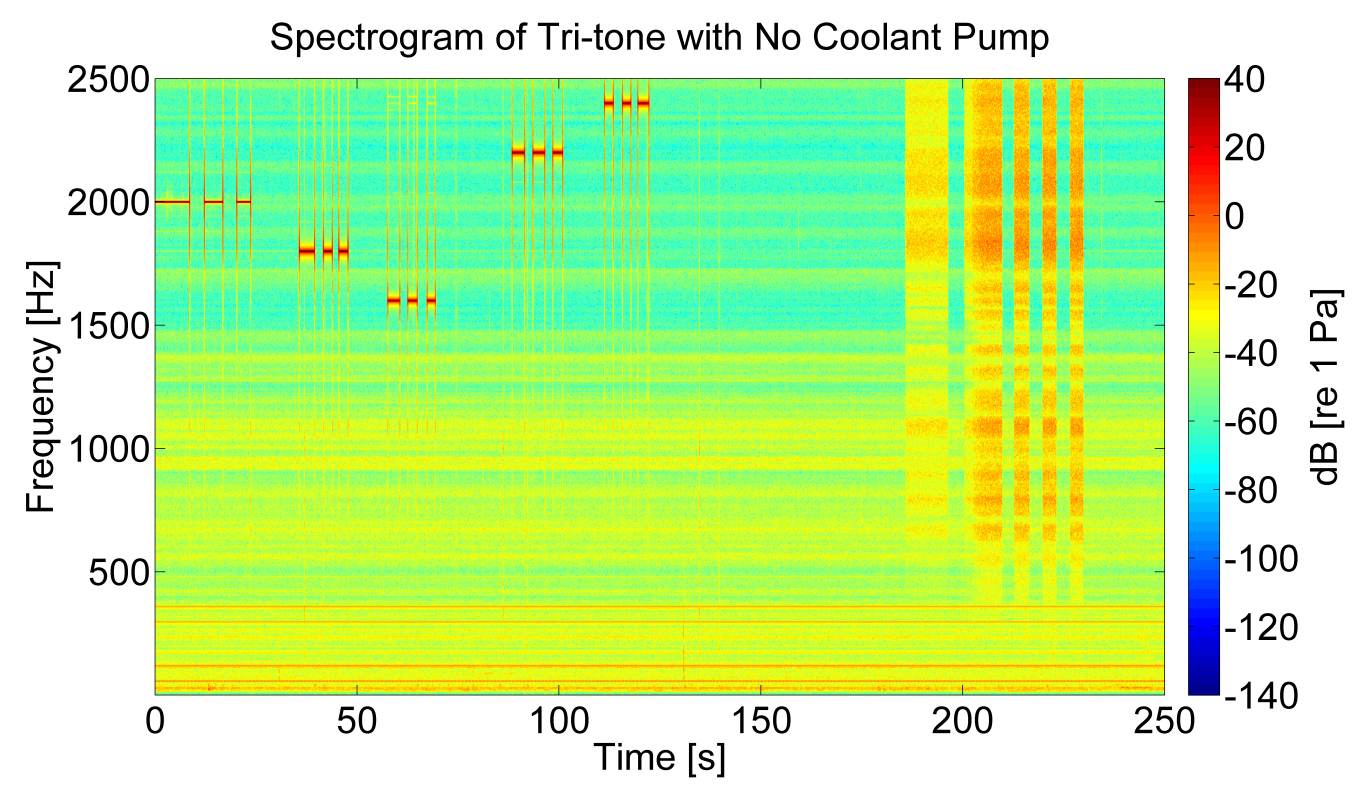

Figure 3.25: Time series spectrogram of H-008 for the reverberation measurements with a 0.37 second $(2.7 \mathrm{~Hz}$ bin) Hann window (no averaging) and $80 \%$ overlap between time records. The change in bin size varied based on visual inspection for smoothing in the spectrogram. Each slice in time represent a separate steady-state excitation and the corresponding cutoff. The first five periods between 0 s and 130 s correspond to excitation at frequencies ranging from $1.6 \mathrm{kHz}-2.4 \mathrm{kHz}$ in steps of $200 \mathrm{~Hz}$. The sixth period from 180s to 230s corresponds to broadband white noise.

\begin{tabular}{|c|c|}
\hline \multicolumn{2}{|c|}{$\begin{array}{c}\text { Reverberation Times } \\
\text { (Hydrophone-007) }\end{array}$} \\
\hline Frequency & $\mathbf{T}_{60}(\mathrm{~ms})$ \\
\hline $1.6 \mathrm{kHz}$ & 36 \\
\hline $1.8 \mathrm{kHz}$ & 8.0 \\
\hline $2 \mathrm{kHz}$ & 12 \\
\hline $2.2 \mathrm{kHz}$ & 22 \\
\hline $2.4 \mathrm{kHz}$ & 51 \\
\hline Broadband & 36 \\
\hline
\end{tabular}

Table 3.1: Reverberation times for hydrophone-007. The low reverberation times suggested that the hydrophone was experiencing direct sound decay from the sound source, rather than representative behavior of the diffuse sound field in the reactor pool. In Sec. 3.2.1, hydrophone-008 is relocated and longer $T_{60}$ values are calculated. 


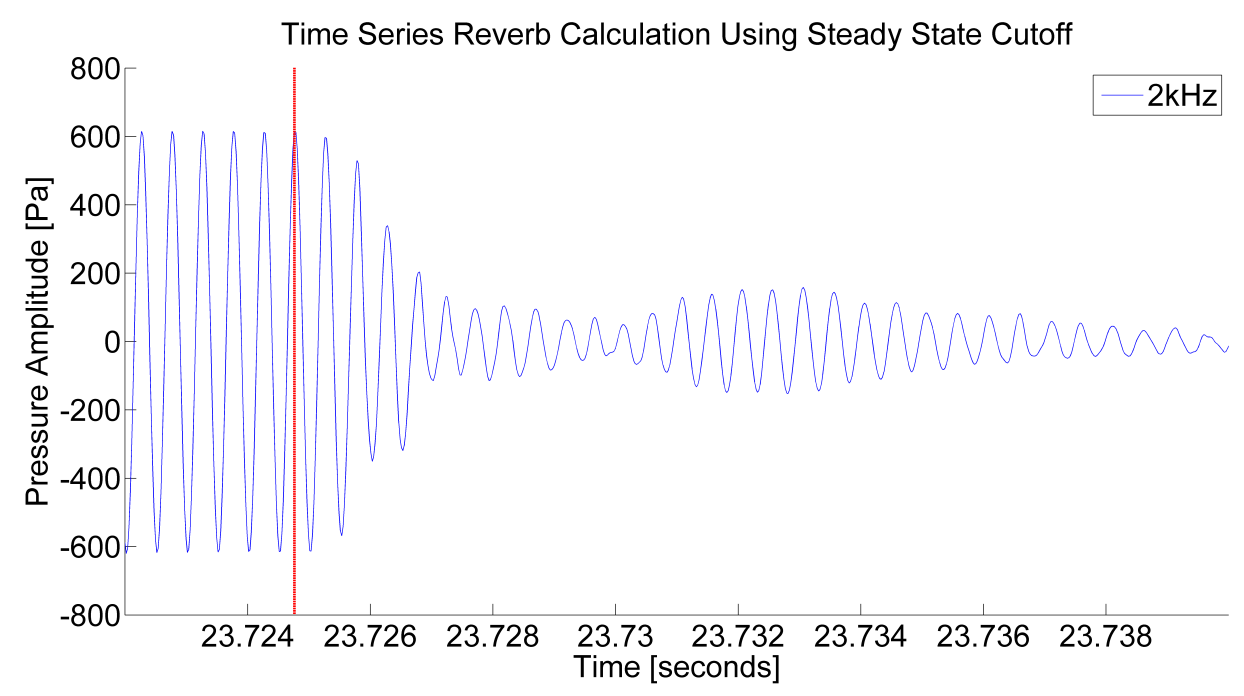

Figure 3.26: An example of the time series cutoff of $\mathrm{H}-007$ during the $2 \mathrm{kHz}$ reverberation measurement. The red line indicates the time when the sound source was cutoff, and everything after is considered the decay and reverberation of the reactor pool. 


\subsection{December 04, 2014}

Further background measurements were taken in the Breazeale reactor using the same two hydrophones, but no accelerometer. Hydrophone-008 was moved to the opposite end of the reactor pool to acquire non-direct sound decay measurements. The new location of H-008 is shown in Fig. 3.2. Two methods of measurement were used: a steady-state cutoff reverberation measurement with five different frequencies $(1.6 \mathrm{kHz}-2.4 \mathrm{kHz}$ in steps of $200 \mathrm{~Hz}$ ); and a linear frequency sweep from $1 \mathrm{kHz}-3 \mathrm{kHz}$. The Lubell underwater sound source and both hydrophones were again used for these measurements. Figure 3.27 illustrates the relocation of hydrophone-008 in the reactor pool.

\subsubsection{Reverberation Measurements After Relocation of Hydrophone-008}

Figure 3.28 shows the time series during the reverberation measurements. Hydrophone008 shows a significantly smaller amplitude than H-007. Since the transmission loss is negligible due to low absorption of sound waves in water (see Eq. 2.36), this decrease in amplitude can be attributed to absorption from the walls of the reactor and other phenomena not fully explored (reflection effects, scattering, etc.).

Hydrophone-008 also shows very high SNR, and the lengthened time decay (see Table 3.2) for each frequency burst validates the original hypothesis that the hydrophone measurements on November 12, 2014 were picking up direct sound from the sound source. Figure 3.31 shows the time series and cutoff point for the $2 \mathrm{kHz}$ reverberation measurement. The longer time window and slower decay represents the diffuse sound field in the pool, rather than the direct transmission from source to receiver.

A spectrogram (Fig. 3.30) was then plotted to demonstrate the ability to monitor frequencies and amplitudes over time. The ability to monitor frequency changes over time is analogous to temperature monitoring over time, something that is critical to reactor monitoring in the nuclear industry. 


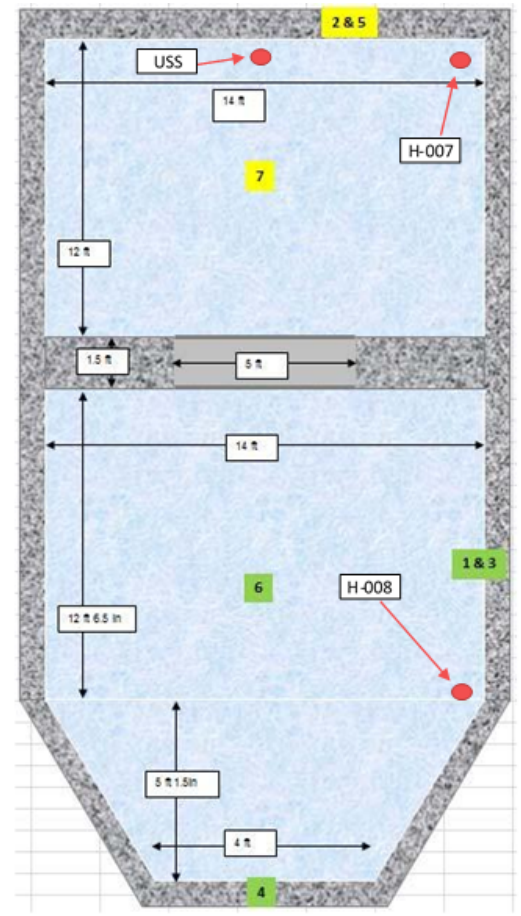

Figure 3.27: Transducer placement in the Breazeale reactor pool during the December 04, 2014 measurements. The red ovals represent the labeled transducer. H-008 has been relocated to decouple it from the direct path to the sound source. Note: the accelerometer was not required for these reverberation time measurements.
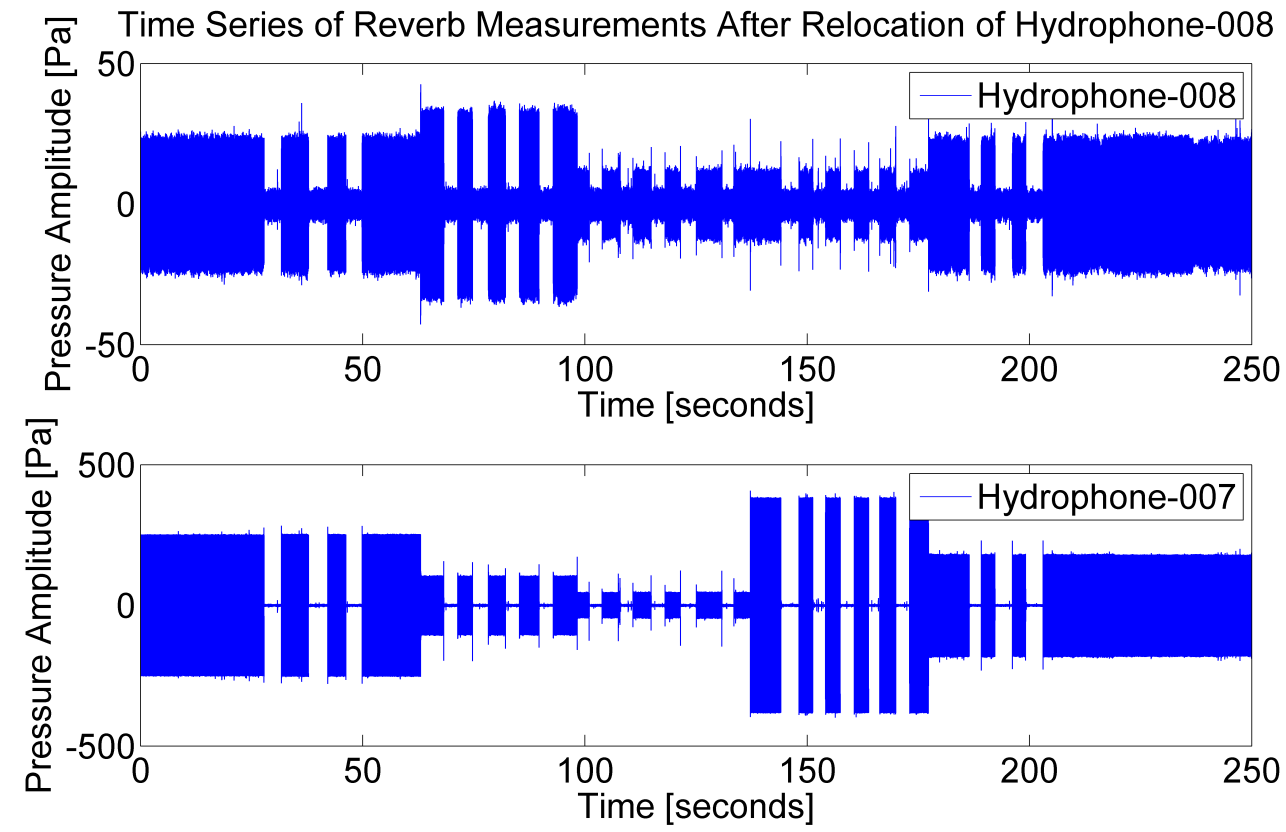

Figure 3.28: Time series for each reverberation segment after H-008 was relocated. Each time interval represents a separate steady-state drive and its corresponding cutoff. The five periods contained frequencies ranging from $1.6 \mathrm{kHz}-2.4 \mathrm{kHz}$ in steps of $200 \mathrm{~Hz}$. 


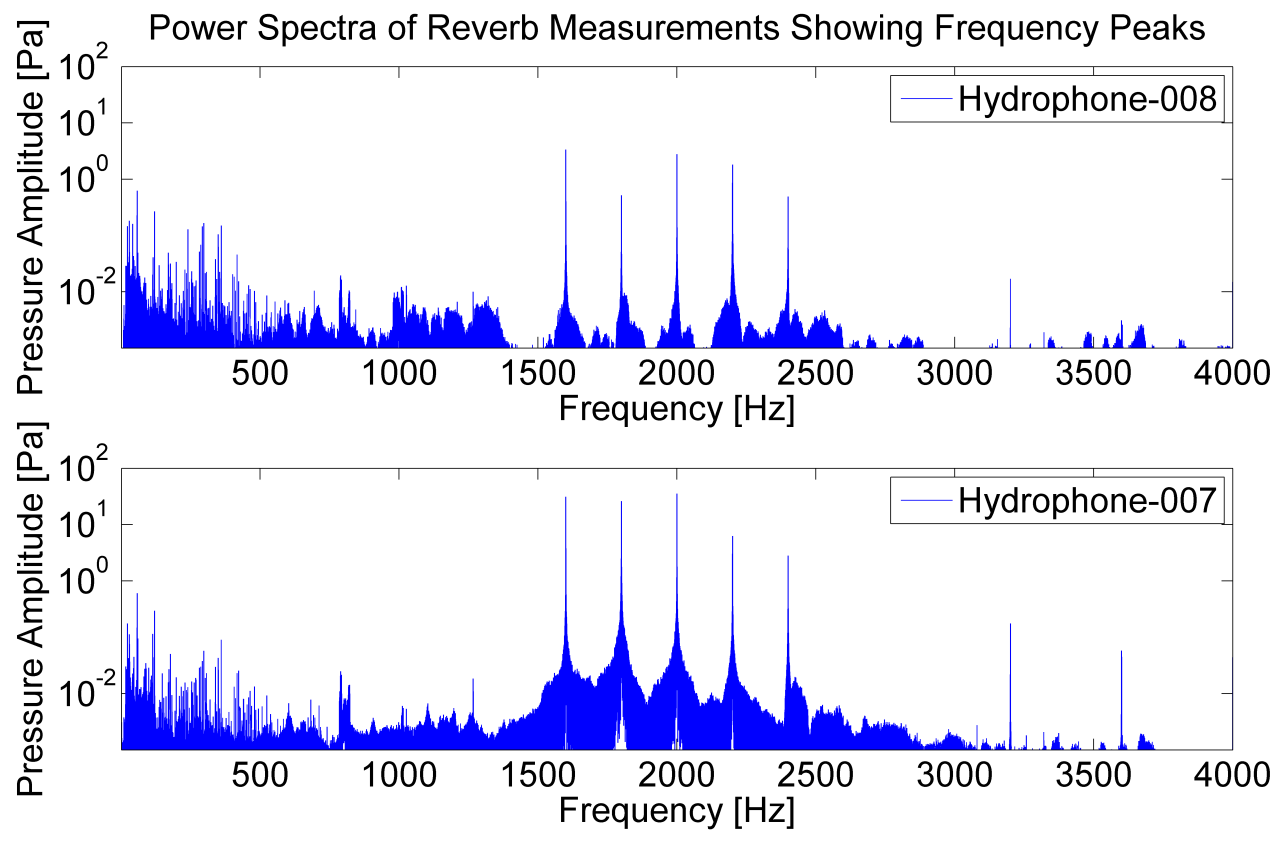

Figure 3.29: Power spectra showing the five frequency peaks from $1.6 \mathrm{kHz}-2.4 \mathrm{kHz}$, in steps of $200 \mathrm{~Hz}$, for all three transducers.

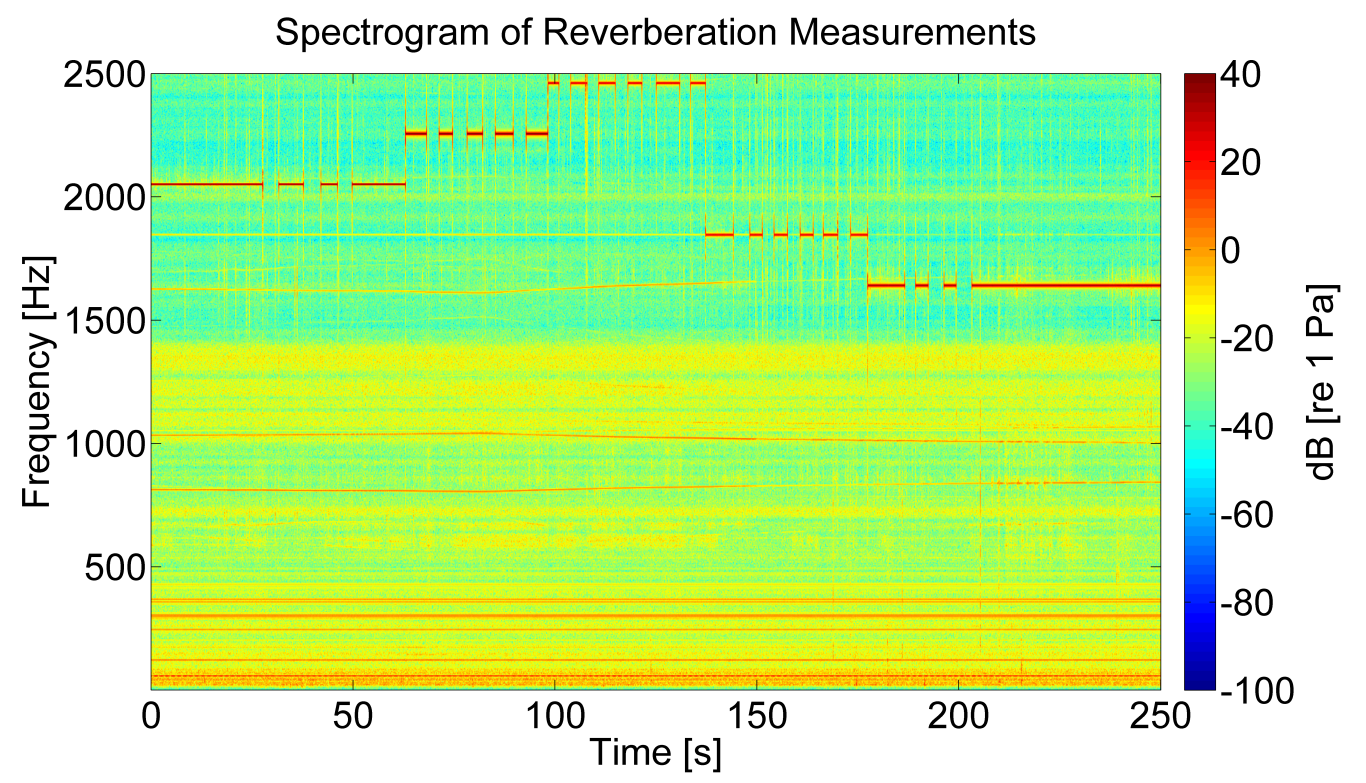

Figure 3.30: Time series spectrogram of $\mathrm{H}-008$ for the reverberation measurements after relocation. A 0.37 second Hann window $(2.7 \mathrm{~Hz}$ frequency bin) with $80 \%$ overlap between time records was used. No averaging between power spectra was done. Each slice in time represents a separate steady-state setup and its corresponding cutoff. The five records were driven at frequencies ranging from $1.6 \mathrm{kHz}-2.4 \mathrm{kHz}$ in steps of $200 \mathrm{~Hz}$. 


\begin{tabular}{|c|c|c|}
\hline \multicolumn{2}{|c|}{$\begin{array}{c}\text { Reverberation Times } \\
\text { (Hydrophone-008) }\end{array}$} \\
\hline Frequency & $\mathrm{T}_{60}$ (ms) & $\begin{array}{c}\text { Critical } \\
\text { Distance (m) }\end{array}$ \\
\hline $1.6 \mathrm{kHz}$ & 168 & 1.08 \\
\hline $1.8 \mathrm{kHz}$ & 54.0 & 1.90 \\
\hline $2 \mathrm{kHz}$ & 200 & 1.00 \\
\hline $2.2 \mathrm{kHz}$ & 180 & 1.04 \\
\hline $2.4 \mathrm{kHz}$ & 120 & 1.28 \\
\hline
\end{tabular}

Table 3.2: Reverberation times and the corresponding critical distances for hydrophone-008 after relocation. The longer reverberation times suggest that this hydrophone experienced effects not fully understood in its previous location, such as low side lobes due to a directional sound source or back-scattering attenuation. Each critical distance is based on the measured reverberation time in the previous column. The average critical distance was $1.26 \mathrm{~m}$. All of the sensors were farther than $1.26 \mathrm{~m}$ from any noise or sound source, therefore concluding that the measurements were made in a diffuse sound field, and it is possible that the original assumption - that low reverberation times were a result of direct sound decay from the sound source - was incorrect.

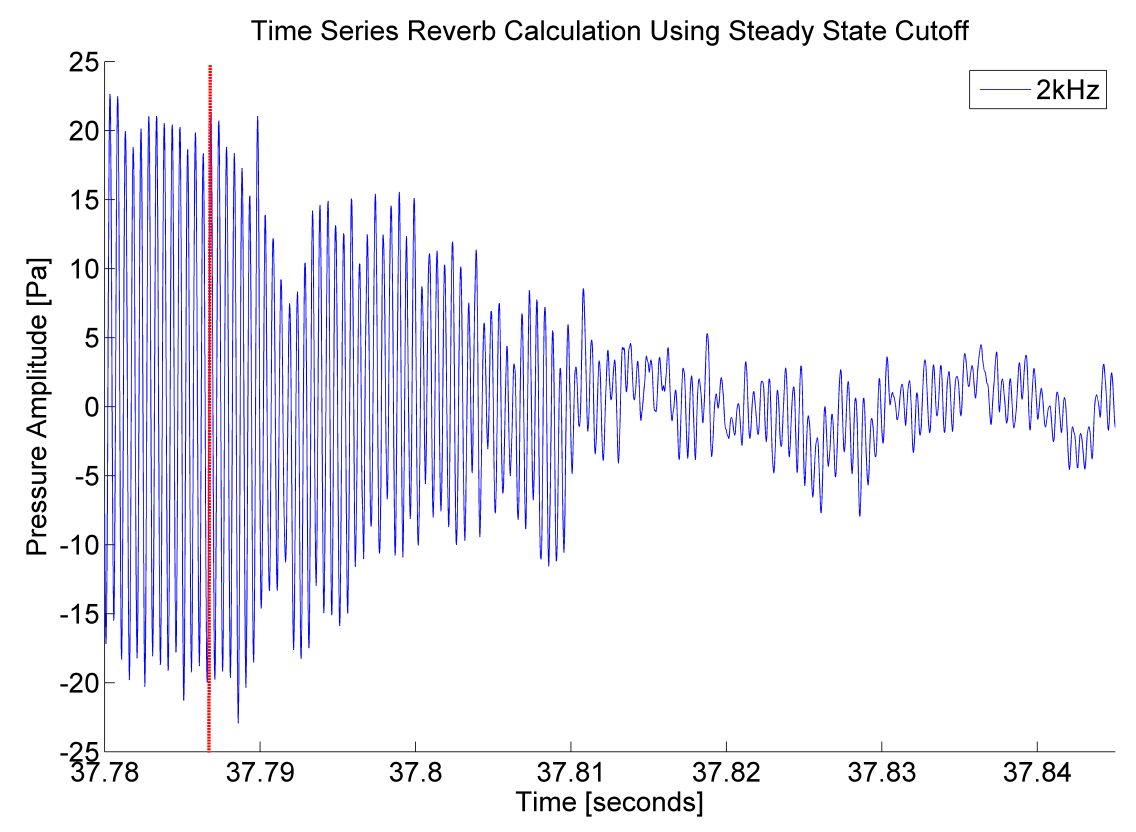

Figure 3.31: Example of the time series cutoff of $\mathrm{H}-008$ during the $2 \mathrm{kHz}$ reverberation measurement after the hydrophone was relocated. The red line indicates the time when the sound source was cut off, and everything after is the decay characterizing the reverberation in the reactor pool. 


\subsubsection{Frequency Sweep Measurement}

A swept-sine signal was provided to the source to mimic the frequency variations over time that would indicate a monotonic increase of coolant temperature, expressed by a TAC sensor, in a nuclear reactor. A Nitrogen-16 $\left({ }^{16} \mathrm{~N}\right.$ or N-16) diffuser pump was active during these measurements. The ${ }^{16} \mathrm{~N}$ diffuser pump is designed to distribute, hence dilute, the radioisotope of Nitrogen produced during normal reactor operation before it can reach the pool surface. This also means that all possible pump noise was present and active during the acquisition of this frequency sweep measurement. Figure 3.32 shows the frequency being swept from $1 \mathrm{kHz}$ to $3 \mathrm{kHz}$ over approximately 3 minutes. Despite the noise introduced by the ${ }^{16} \mathrm{~N}$ pump, the high SNR confirms the potential for reactor temperature monitoring. The amplitude and frequency tracking will be explored later in Sec.

3.3.3 when the spectrogram and power spectrum are compared with one another.

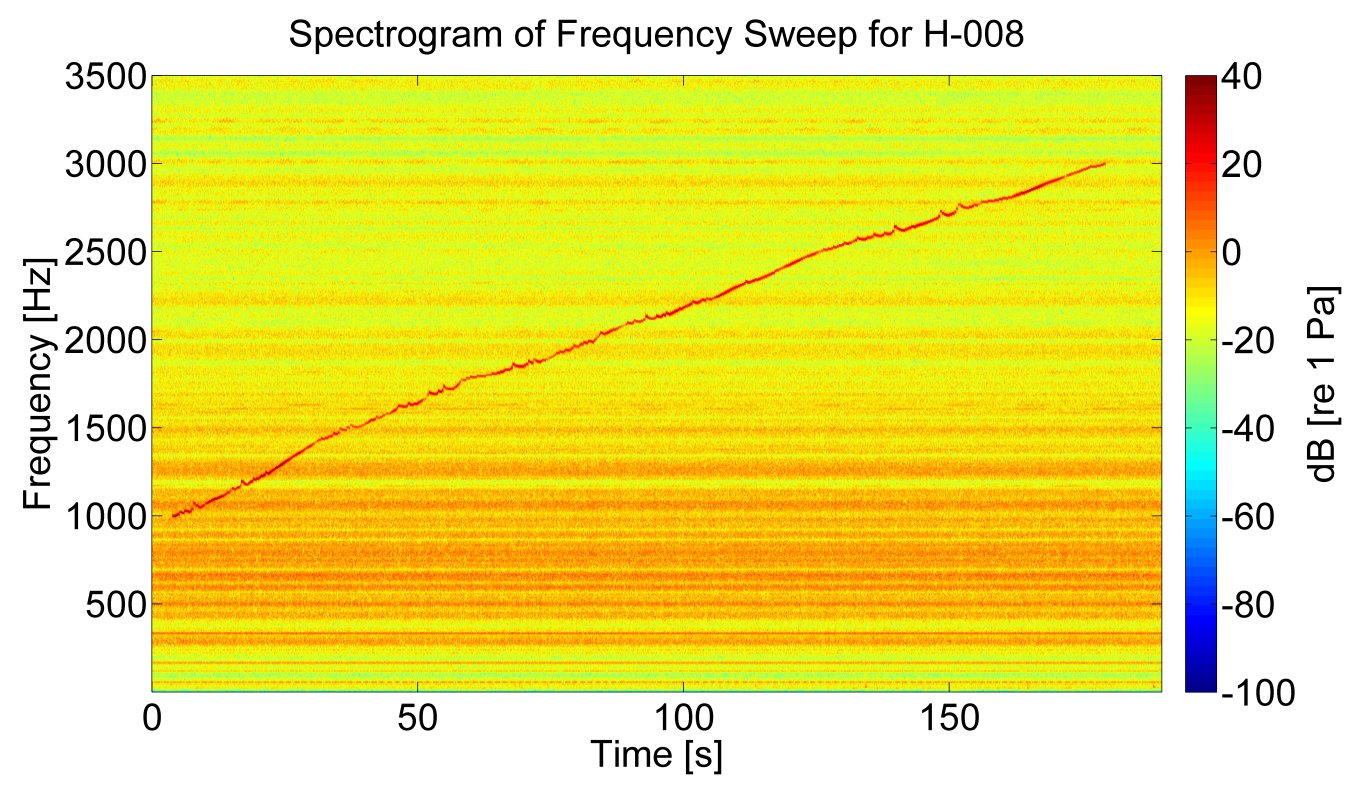

Figure 3.32: Time series spectrogram of H-008 for the swept sine measurement after relocation. A 0.186 second long $(5.38 \mathrm{~Hz}$ bin) Hann window (no averaging) and $95 \%$ overlap was used. $95 \%$ overlap with a 0.186 second long window was used to capture enough cycles of the frequencies of interest, but smooth them enough to be able to observe subtle changes with high time resolution, which will be important for quick changing processes such as a frequency sweep. Subsequently, the sound source was swept from $1 \mathrm{kHz}$ to $3 \mathrm{kHz}$ to simulate a rapid rise in coolant temperature. The clarity of the sweep is a good indicator of the ability to monitor temperature in a nuclear reactor. Even in the low frequency end amidst the pump noise $(\mathrm{f}<1.5 \mathrm{kHz})$, a clear signal evident at the chosen drive level.

\subsection{Reverberation Analysis and Frequency Filtration}

This section provides examples of the reverberation time analysis that produces the values of $T_{60}$ in Table 3.2. There is also a brief calculation of the propagation delay between 
hydrophone-007 and hydrophone-008 that demonstrates that the paths are understood. Pump noise filtering and measurement display techniques are then presented in Sec. 3.3.2.

\subsubsection{Reverberation Time and Direct Sound Decay}

$T_{60}$ values were measured once Hydrophone-008 was relocated across the pool. For room acoustics in air, the standard for calculation of reverberation time involves terminating the sound emission source, and calculating the time it takes to drop from $5 \mathrm{~dB}$ below its peak value to either $25 \mathrm{~dB}$ below or $35 \mathrm{~dB}$ below that peak value [11]. Unfortunately, for the measurements in the Breazeale reactor pool, noise levels were so high that the maximum signal amplitude never exceeded $15 \mathrm{~dB}$ above the noise level. As a result of this, decibel levels vs. time were plotted on a linear axis and fitted with a straight line to calculate a single $T_{60}$ value. This would emulate the extrapolation technique used in room acoustics for $20 \mathrm{~dB}$ and $30 \mathrm{~dB}$ drops [16]. An example of this process is shown in Figs. 3.33 and 3.34 using the $2 \mathrm{kHz}$ tone. Figure 3.33 shows a longer time record for the same event as shown in 3.34. The decay was quite complex for many of the cutoff records, and as a result the $T_{60}$ values are only approximate. The slope that accounted for the most linear decay was used to determine the reverberation time for each measurement. After fitting a straight line to the decay, the slope of that line is divided by 60 to obtain the time it takes for a signal to decay $60 \mathrm{~dB}$. The values in Table 3.2 were calculated using this approach.

The average $T_{60}$ value was roughly $144 \mathrm{~ms}$ with a standard deviation of $58 \mathrm{~ms}$, representing a standard error of about $40 \%$. This error will propagate into the calculations for the minimum power output needed for a detectable pressure amplitude. Since the $T_{60}$ value is under a square root with the power term (see Eq. 2.43), the error could be as high as $65 \%$. However, the error propagation is not large enough that it is of concern in the calculations of the predicted pressure amplitude. This is due to the highly variable nature of the nuclear power, and the approximations made under countless variables included in the resonator physics (See Ref. [21]). 


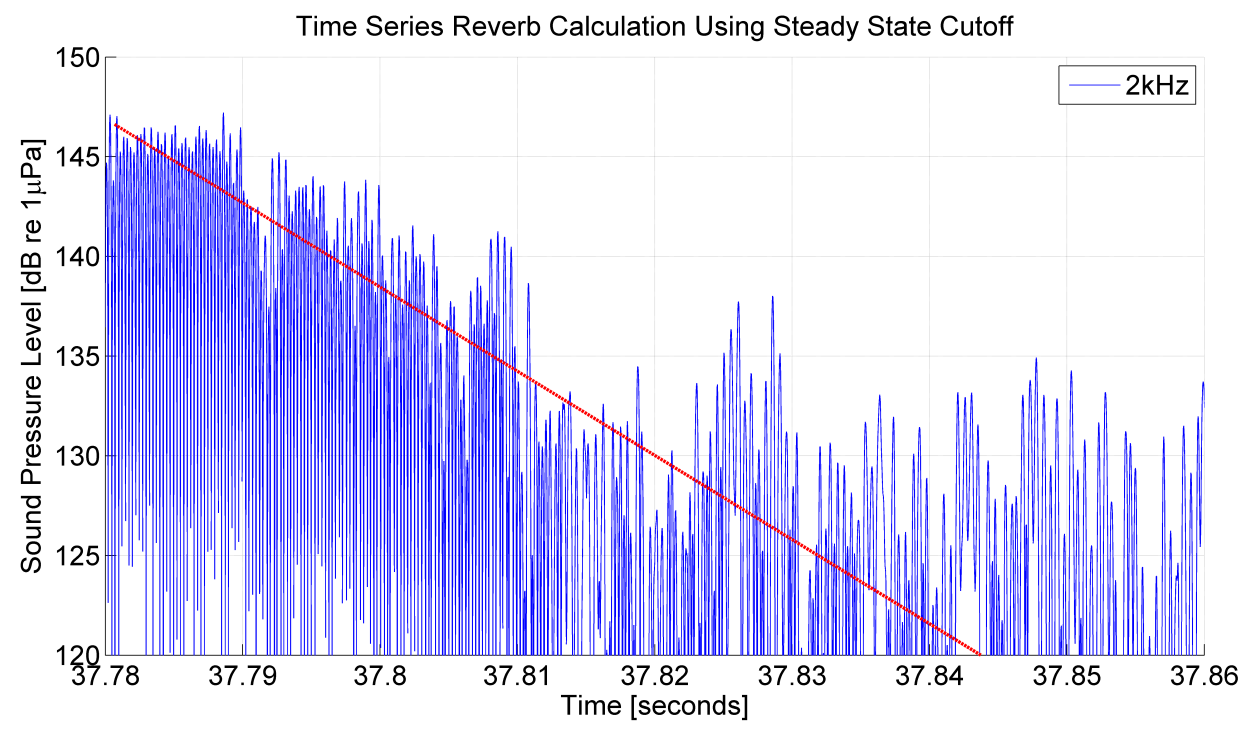

Figure 3.33: Example of the decibel level vs. time cutoff method applied to the signal from $\mathrm{H}-008$ during the $2 \mathrm{kHz}$ reverberation measurement after the hydrophone was relocated. The line approximates the slope of the energy decay. The slopes were approximated by using a slope-intercept method at two different points on the decay curve.

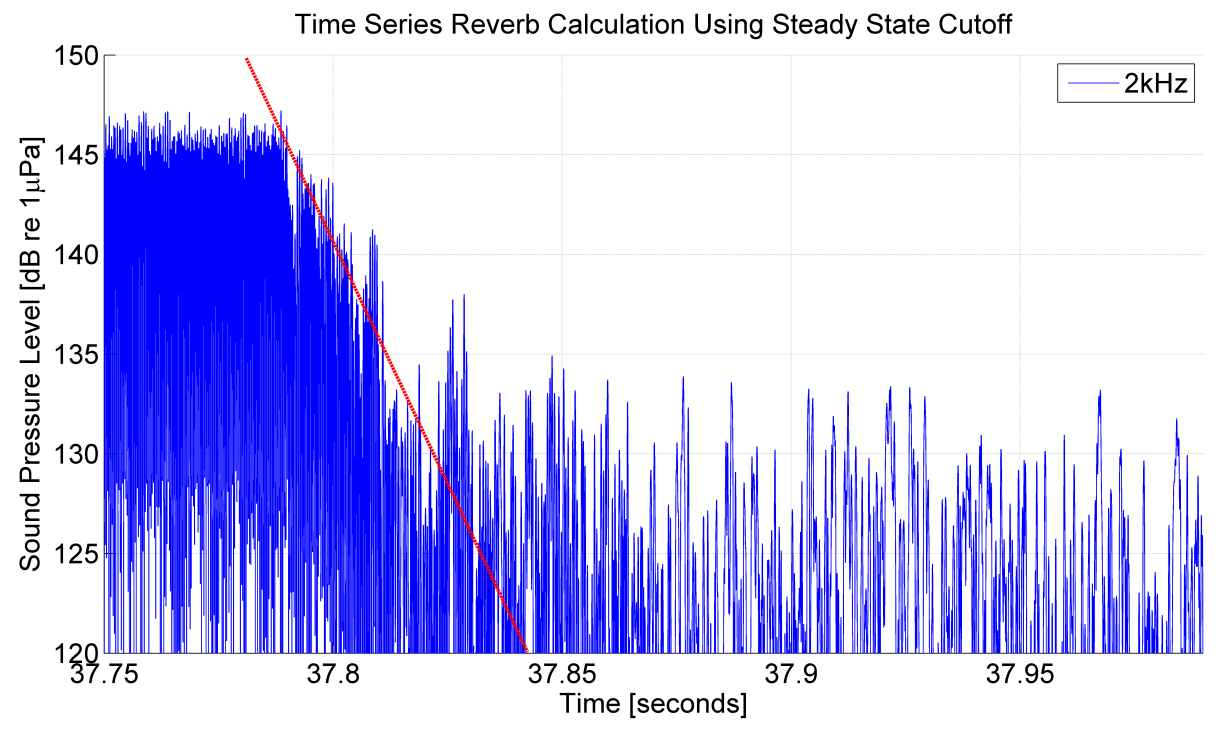

Figure 3.34: A longer view of the time series cutoff for $\mathrm{H}-008$ during the $2 \mathrm{kHz}$ reverberation measurement (after hydrophone-008 was relocated). The line indicates the slope of the amplitude decay after the cutoff that characterizes the losses in the pool.

\subsubsection{Filter Techniques}

After the inspection and study of the pump noise from Secs. 3.1 and 3.2, it is evident that the majority of interference introduced by the pumps remains below $1 \mathrm{kHz}$ for the hydrophones. Above $1.5 \mathrm{kHz}$, the noise floor remains fairly constant. Figure 3.35 clearly il- 


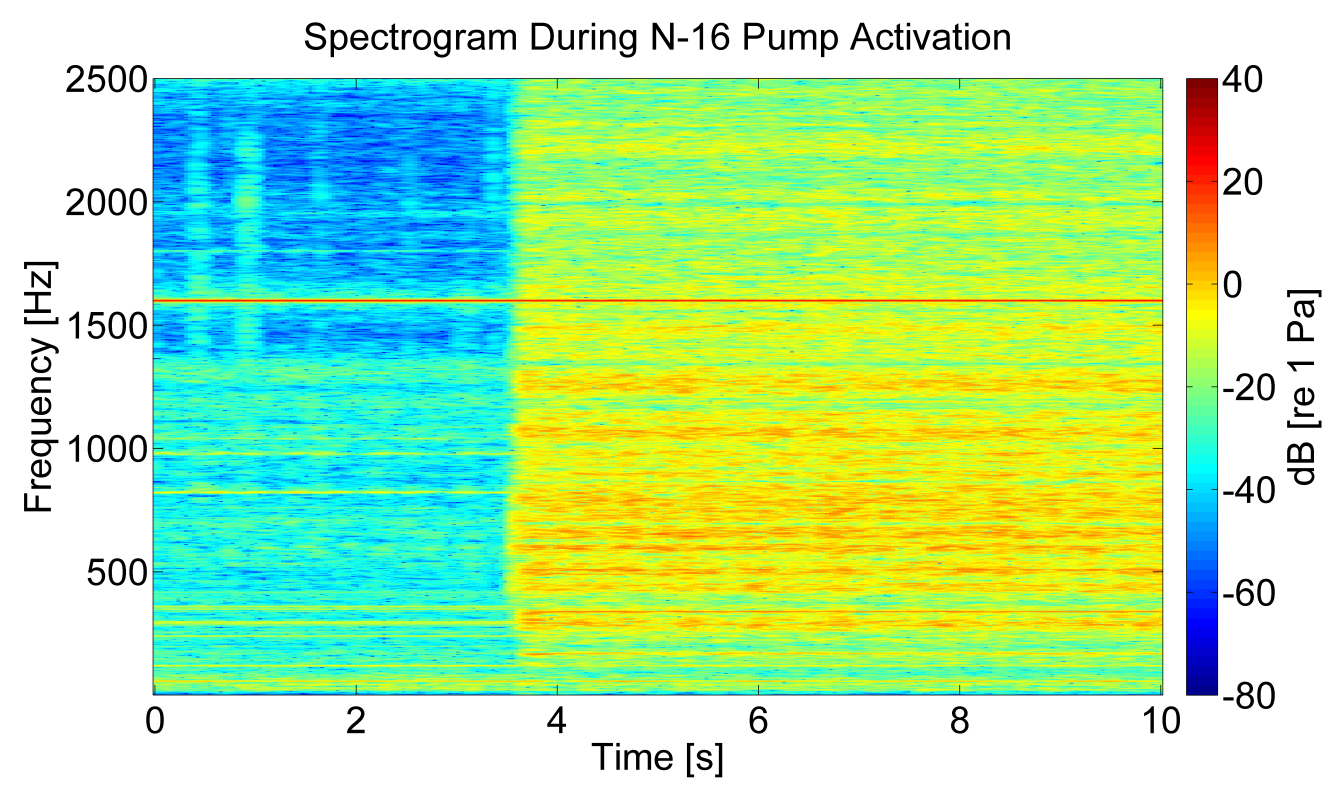

Figure 3.35: Time series spectrogram of $\mathrm{H}-008$ during the activation of the ${ }^{16} \mathrm{~N}$ diffuser pump. A 0.37 second $(2.7 \mathrm{~Hz}$ bin) Hann window (no averaging) and $90 \%$ overlap of time records was used. The sound source was generating a $1.6 \mathrm{kHz}$ sine wave when the pump was activated. The activation of the pump is seen right before the 4 second mark. Notice the low frequency content of the pump signal. The frequency content after the 4 second mark is representative of the largest amount of noise during normal reactor operation.

lustrates the change in background noise upon activation of the ${ }^{16} \mathrm{~N}$ diffuser pump. Hydrophone008 is within a few feet of the diffuser outlet pump and still manages to resolve a clear tonal. In Fig. 3.36 a bandpass filter was used on the same data set. Comparison of the two reveals a more uniform background noise level due to filtration. Figure 3.36 utilized a 3rd order, bandpass, butterworth filter with a cutoff at $1.2 \mathrm{kHz}$ and $20 \mathrm{kHz}$. The cutoff frequency smoothly transitions between attenuation and cut-on. Consequently, almost no amplitude reduction is introduced at the $1.6 \mathrm{kHz}$ tone. The butterworth filter cutoff was chosen because of its minimal effects on the $1.6 \mathrm{kHz}$ tonal amplitude. Figure 3.37 shows the response for three different conditions: no filter, a simple single-pole highpass filter (with cutoff at $1.2 \mathrm{kHz}$ ), and the 3 rd order butterworth bandpass filter. The bandpass filter is a good idea in this case because the interesting frequencies lie within a few $\mathrm{kHz}$ of $1.5 \mathrm{kHz}$. There is less opportunity for electrical power mains noise $(60 \mathrm{~Hz})$ or pump tonals to interfere with the analysis when those (primarily) low frequency components are filtered out. A passive highpass filter will likely be added between the analog ouput of the hydrophones preamplifier and the recording device. This configuration permits the increase of the input sensitivity of the recorder without risking saturation due to large amplitude, low-frequency pump noise.

After attenuation of low frequency noise, narrow-band single-frequency filtering is explored. An "octave filter" specifies a center frequency, calculates the two frequencies above 


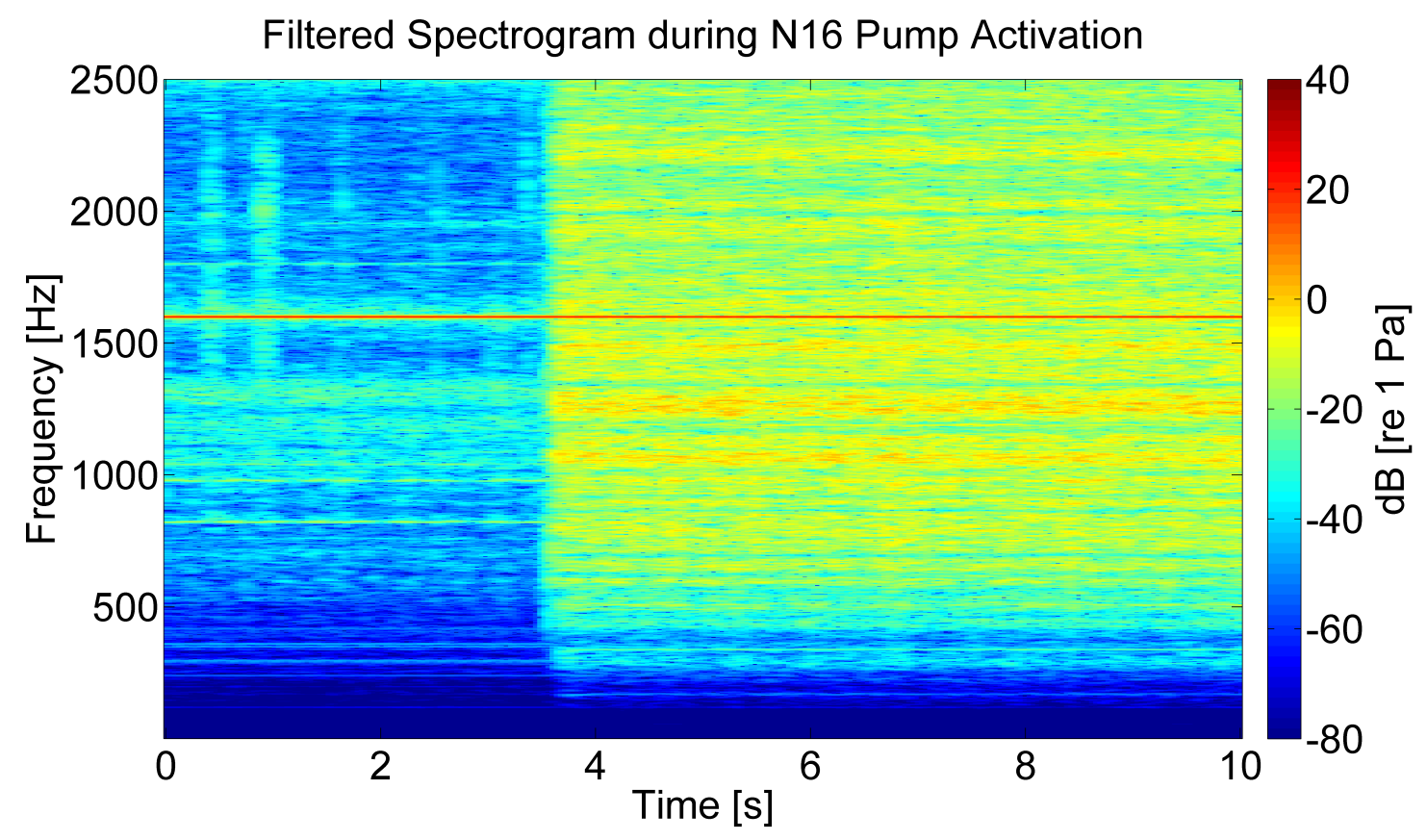

Figure 3.36: Low-frequency filtered time series spectrogram of $\mathrm{H}-008$ during the activation of the ${ }^{16} \mathrm{~N}$ diffuser pump. A 0.37 second $(2.7 \mathrm{~Hz}$ bin) Hann window (no averaging) and $90 \%$ overlap of time records was used, as well as a 3rd order butterworth bandpass filter that rolls off at $1.2 \mathrm{kHz}$ and $20 \mathrm{kHz}$. The sound source was generating a $1.6 \mathrm{kHz}$ sine wave while the pump was activated. When compared to Fig. 3.35, the effect of the filter makes the background noise nearly independent of frequency.

and below it that constitute an "octave," and implement a bandpass filter over that range. A similar method was used with the center frequencies chosen above $(1.6 \mathrm{kHz}-2.4 \mathrm{kHz})$, but a $1 / 15$-octave bandwidth was utlitized to better isolate tonal frequencies from neighboring peaks. Figure 3.38 shows a $1 / 15$-octave filter applied to the $1.6 \mathrm{kHz}$ reverberation measurement after relocation of $\mathrm{H}-008$ in Sec. 3.2.1. A peak-detection algorithm was also used to isolate single frequencies with amplitudes above a certain threshold. This analysis emulated five possible acoustic resonators, each with a different frequency and amplitude. The ability to distribute resonators with different frequencies allows simultaneous monitoring of more than one region in a reactor core. An individual frequency and its amplitude could telemeter information about temperature and flux to a reactor operator, indicating performance and safety conditions.

Table 3.3 compares the amplitudes of each frequency peak before and after the $1 / 15$ octave filter implementation. The amplitude attenuation could be corrected using signal processing techniques, but for the current analysis only the amplitude ratio is considered before and after the filtering. 


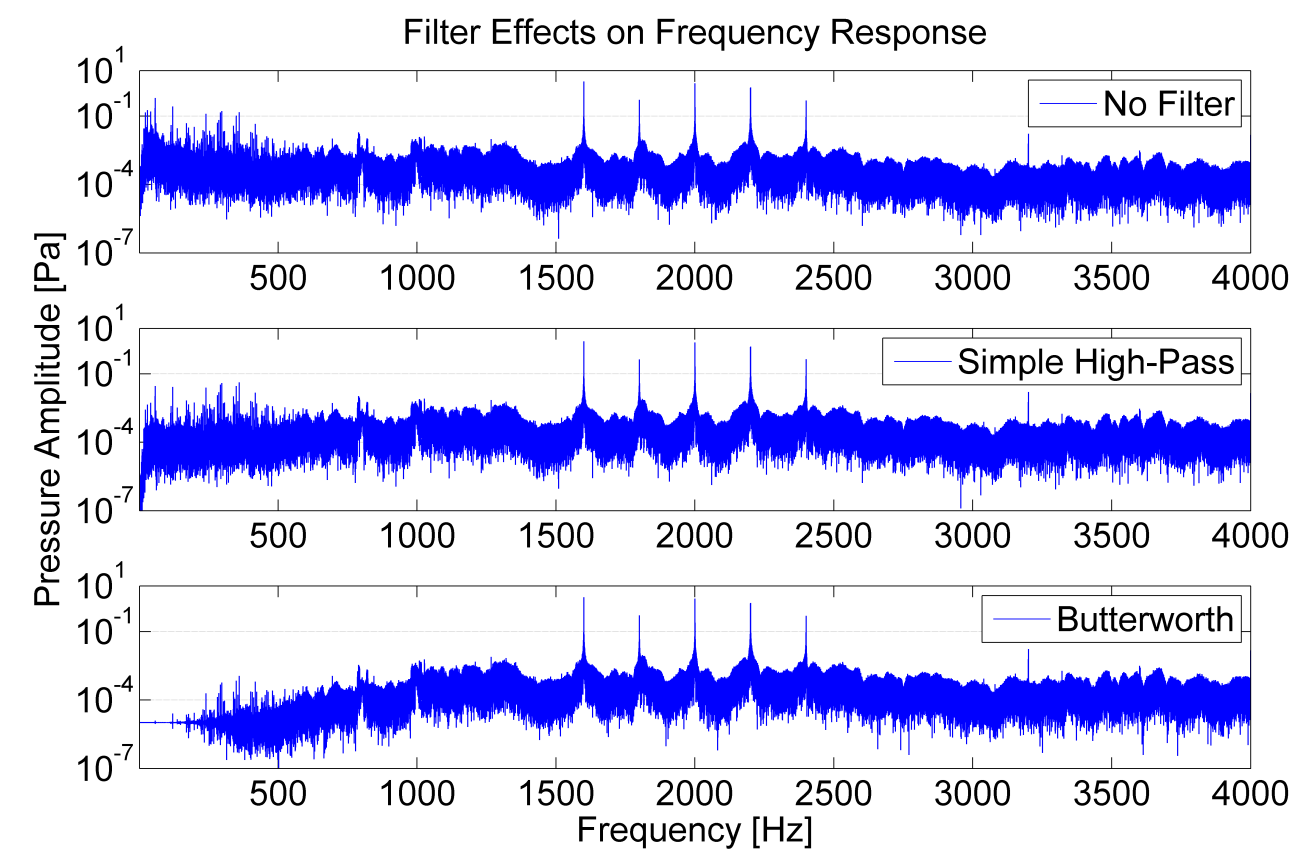

Figure 3.37: Comparison of different filters for the power spectra of the five frequency peaks $(1.6 \mathrm{kHz}-2.4 \mathrm{kHz})$ during reverberation measurements of $\mathrm{H}-008$. The first plot at the top is the unfiltered power spectrum. The plot in the middle is a simple single-pole high-pass filter with a $-3 \mathrm{~dB}$ cutoff frequency at $1.2 \mathrm{kHz}$, and the last plot is a butterworth bandpass filter that has cutoffs at $1.2 \mathrm{kHz}$ and $20 \mathrm{kHz}$.

\begin{tabular}{|c|c|c|c|}
$\begin{array}{c}\text { Amplitude } \\
\text { Attenuation due } \\
\text { to } \begin{array}{c}\text { Amplitude } \\
\text { Filtering }\end{array}\end{array}$ & \multicolumn{2}{|c|}{ Actave } \\
\hline Frequency & Before & After & After/Before \\
\hline $1.6 \mathrm{kHz}$ & 4.55 & 3.10 & 0.68 \\
\hline $1.8 \mathrm{kHz}$ & 1.24 & 0.50 & 0.40 \\
\hline $2 \mathrm{kHz}$ & 4.98 & 2.74 & 0.55 \\
\hline $2.2 \mathrm{kHz}$ & 4.60 & 1.79 & 0.39 \\
\hline $2.4 \mathrm{kHz}$ & 1.17 & 0.49 & 0.42 \\
\hline
\end{tabular}

Table 3.3: Amplitude comparison before and after 1/15-octave filtering. The amplitude attenuation can be reduced by increasing the filter bandwidth, but only a simple peakdetection algorithm and narrow filter are used here. 


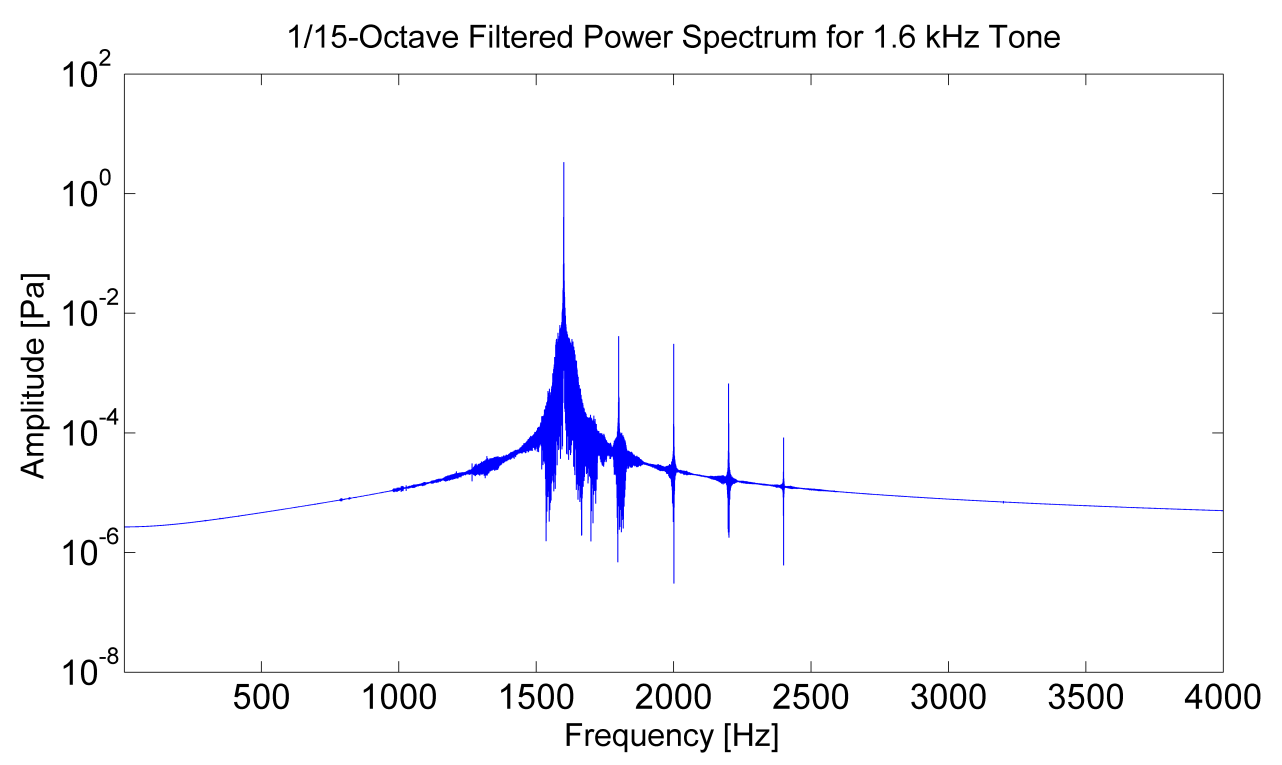

Figure 3.38: $1.6 \mathrm{kHz}$ signal after narrow-band filtering. The amplitude has been attenuated (see Table 3.3). This method of $1 / 15$-octave filtering can be used to track relative amplitudes of a specific frequency as it changes with time.

\subsubsection{Spectrogram vs. Power Spectrum}

A pure tone is characterized by only two parameters: its frequency and its amplitude. Frequency changes can be monitored on a spectrogram, and the amplitude can be tracked using a power spectrum. Real-time measurements may become complicated since a power spectrum requires a time record of non-zero length. Figure 3.39 shows the swept-sine power spectrum output from $1 \mathrm{kHz}-3 \mathrm{kHz}$ (Sec. 3.2.2). Determination of frequency resolution will play an integral role in the resolvable temperature resolution, however, for the current analysis visual and temporal resolution were prioritized to mimic the needs of a reactor operator monitoring a TAC signal in real time. There is no direct indication that a tonal signal is present in the power spectrum data of Fig. 3.39 since the frequency is changing continuously. However, Fig. 3.40 utilizes a spectrogram to depict the time and frequency information simultaneously.

A power spectrum provides a two-dimensional snapshot in time of the frequency and amplitude, but it is not capable of tracking the signal's evolution over time. A spectrogram like the one in Fig. 3.40, on the other hand, monitors frequency variations quite clearly. The spectrogram is also capable of following amplitudes using its colorbar. This can be seen in Fig. 3.41, where the power spectrum and spectrogram are plotted above one another.

The pump activation measurement is a prime example why both power spectrum and spectrogram are complementary. The amplitude of the peak would be difficult to resolve without the power spectrum, and any complex frequency behavior produced by the pumps would be difficult to characterize without the spectrogram. Figure 3.42 shows the com- 


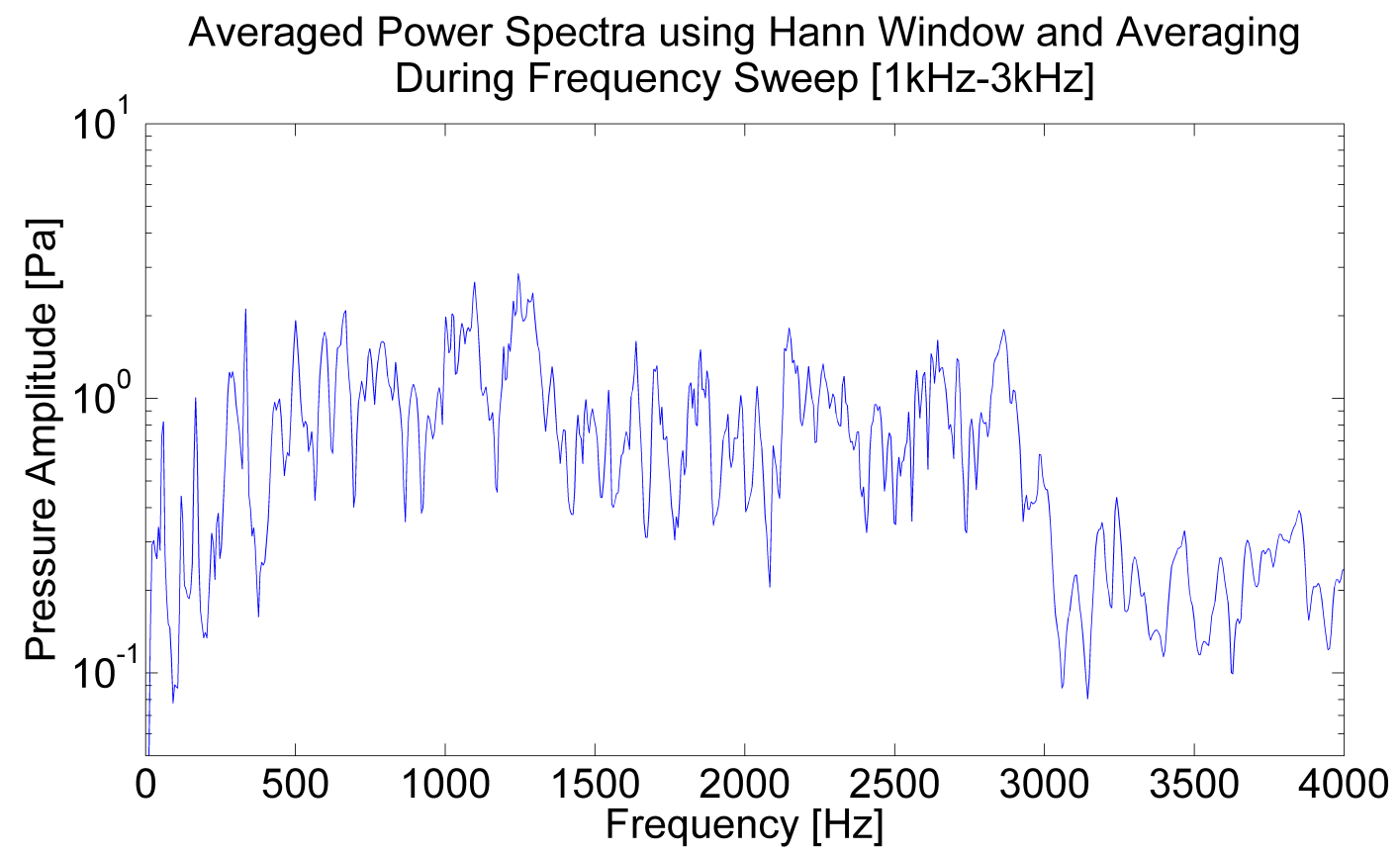

Figure 3.39: Power spectrum showing the indistinct frequency sweep. There is no clear differentiation between the noise and the energy of the signals because the frequency of the tonal is increasing continuously during acquisition of the time record.

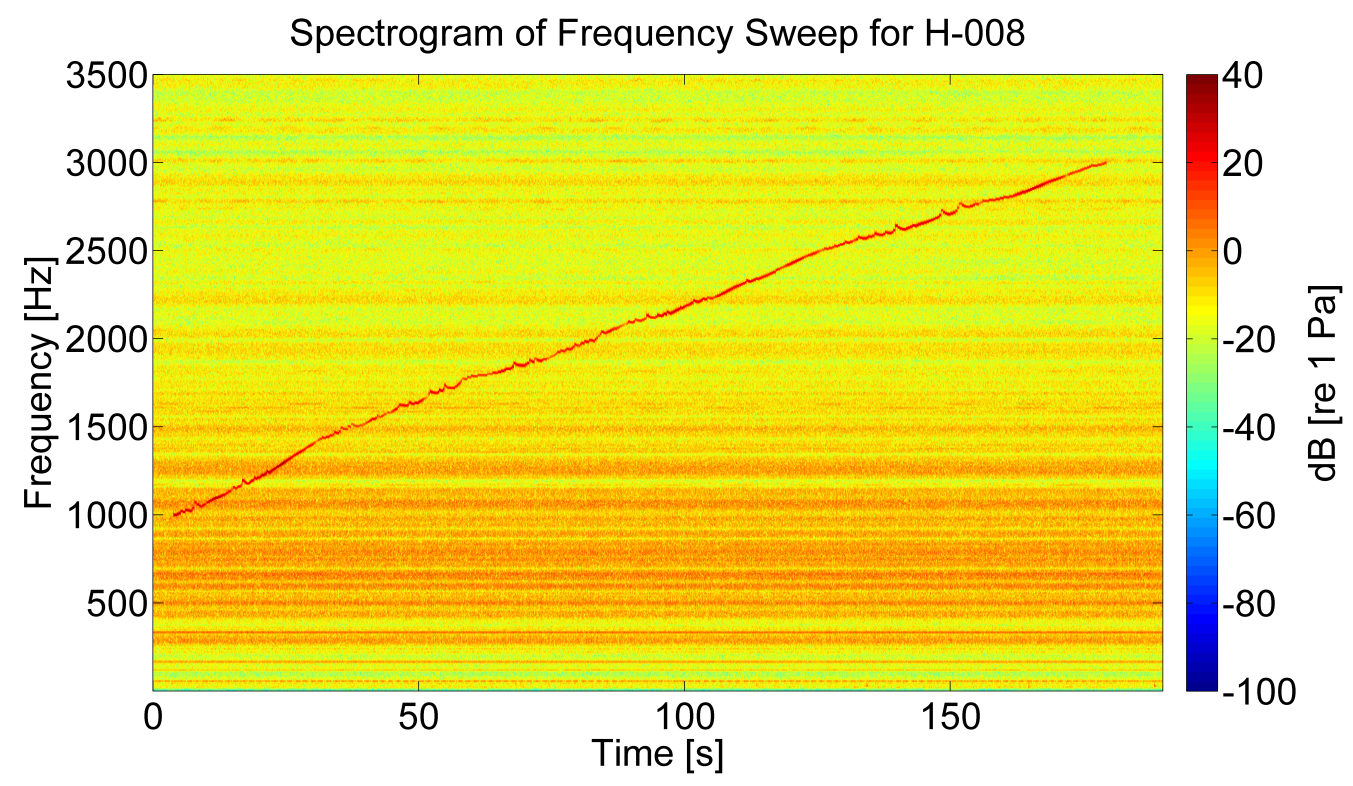

Figure 3.40: Spectrogram of a frequency sweep from $1 \mathrm{kHz}-3 \mathrm{kHz}$. The spectrogram functions as a better method for illustrating frequency changes, whereas the power spectrum is not useful if there are significant frequency changes during acquisition of the time record.

bination of both using a waterfall plot; however, pinpointing particular data on this plot is quite difficult. A 3-D interactive waterfall plot could be particularly useful in the application of monitoring all three variables: time, amplitude, and frequency. 

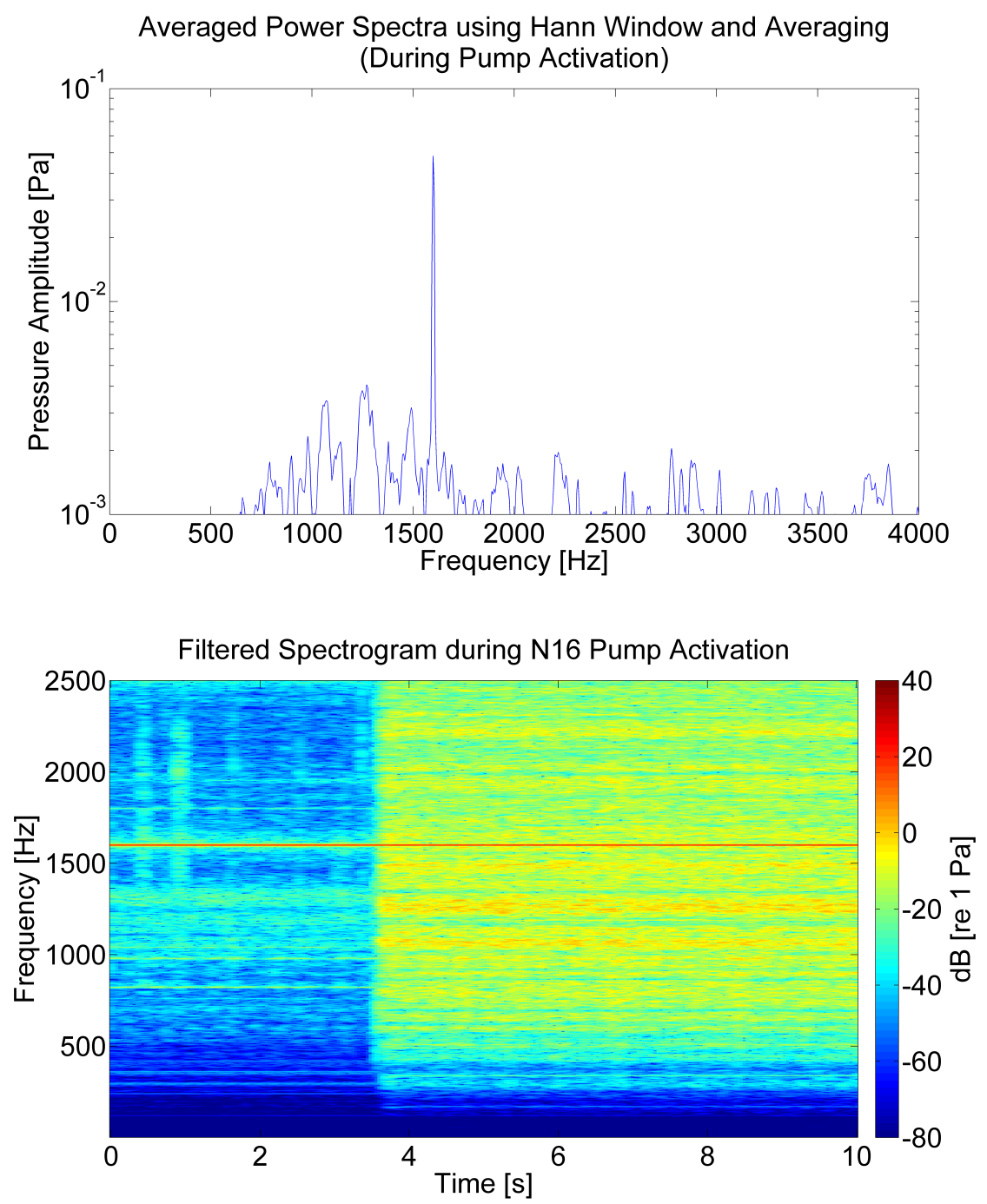

Figure 3.41: Top: power spectrum during the ${ }^{16} \mathrm{~N}$ pump activation. Notice that the lack of time discrimination makes it nearly impossible to see that the pump was activated within the measurement period. Bottom: spectrogram showing the frequency behavior over time. It is easy to see that there was a broadband disturbance that started around the 3.5 second mark. Both power spectrum and spectrogram are important for the tracking and monitoring of acoustic signals. 


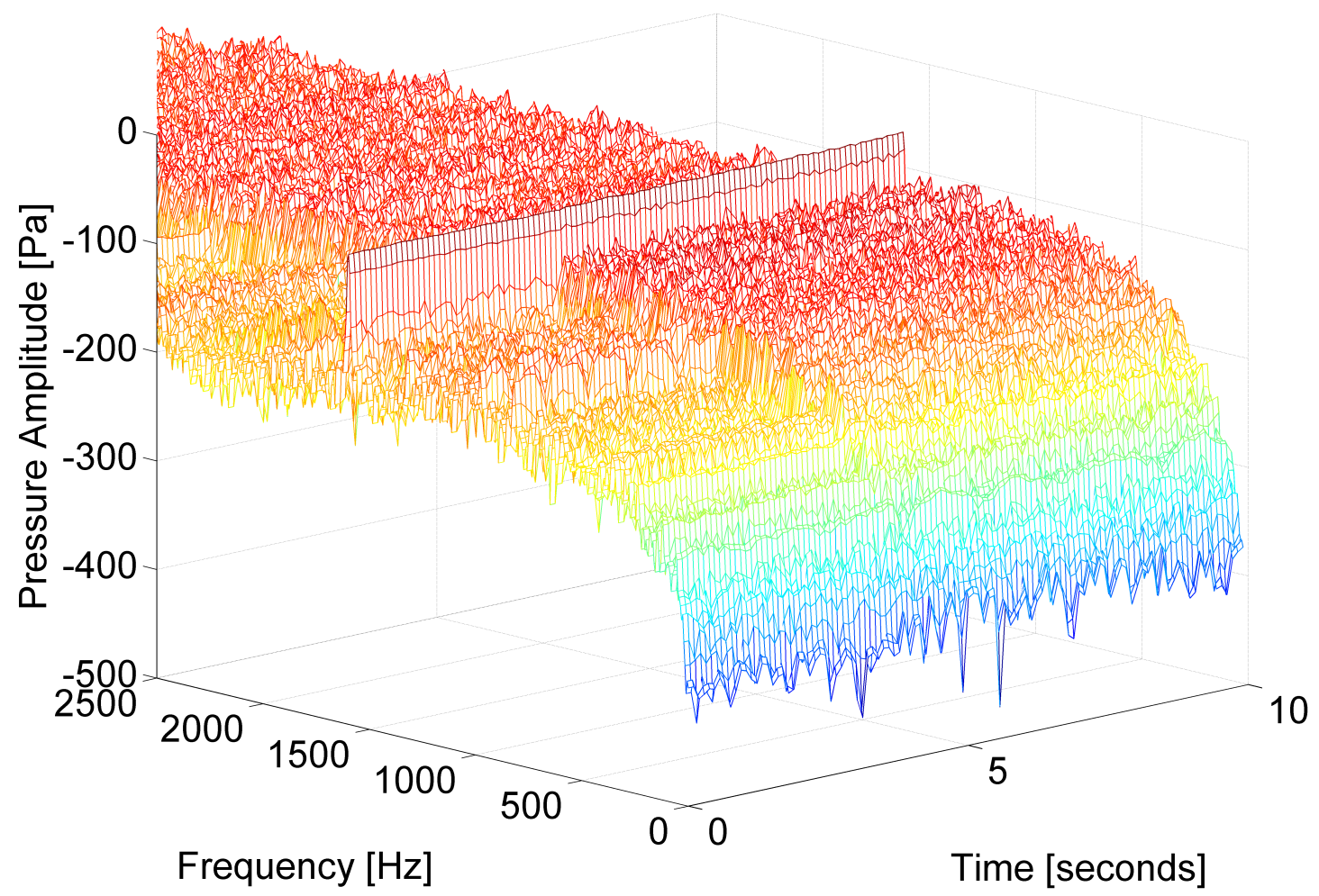

Figure 3.42: Waterfall plot showing the ${ }^{16} \mathrm{~N}$ diffuser pump activation. The waterfall method shows frequency, time, and amplitude values. This type of plot could be useful to a reactor operator monitoring the reactor in real time looking for simultaneous frequency-amplitude changes over time.

\subsubsection{Propagation Delay Between Hydrophones}

After relocating hydrophone-008 across the length of the pool, the propagation time between hydrophones was calculated using the reverberation results from Sec. 3.2. Figure 3.43 represents the propagation path from the underwater sound source to hydrophones -007 and -008. Using the known speed of sound in water, the distance between path A and $\mathrm{B}$ can be used to calculate the approximate value for the time delay between both hydrophones, verifying that a direct path of propagation exists from the underwater sound source to each hydrophone. The time delay between the two hydrophones can be calculated using the simple relation:

$$
\tau=\frac{\Delta d}{v_{\text {water }}}
$$

The variable $\tau$ (not to be confused with Eq. 2.28 in Sec. 2.3.1) represents the time delay between hydrophone-007 and hydrophone-008. The $\Delta d$ is the path length difference from the sound source to $\mathrm{H}-007$ and $\mathrm{H}-008$, and the speed of sound in water at $T=20^{\circ}$ is 


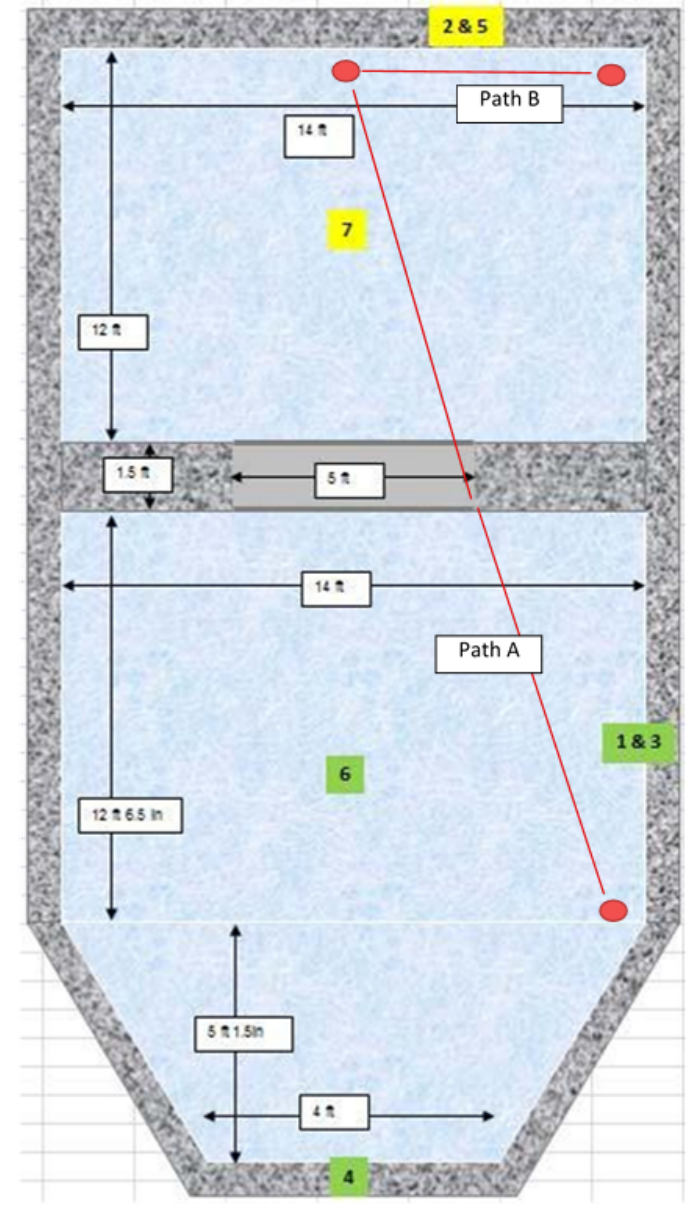

Figure 3.43: Sound propagation path in the Breazeale reactor pool. Path A shows the direct path from the underwater sound source to hydrophone-008 (after relocation), and Path B shows the direct path from the sound source to hydrophone-007. The propagation distances are approximated for each path in Eqs. 3.2 and 3.3.

given by $v_{\text {water. }}$. The variables can be approximated from Fig. 3.27:

$$
\begin{gathered}
d_{\text {uss } \rightarrow 007} \approx 6 \mathrm{ft} \approx 1.83 \mathrm{~m} \\
d_{u s s \rightarrow 008} \approx 27 \mathrm{ft} \approx 8.23 \mathrm{~m} \\
v_{\text {water }}=1481 \frac{\mathrm{m}}{\mathrm{s}} \\
\tau=\frac{d_{\text {uss } \rightarrow 007}-d_{\text {uss } \rightarrow 008}}{v_{\text {water }}} \approx-4.32 \mathrm{~ms}
\end{gathered}
$$

The exact delay is difficult to determine even with the cutoff method from the time series data. Figures 3.44 and 3.45 suggest that a time delay of $4.8 \mathrm{~ms}$ results in an overlay of the time records of the two hydrophones. This delay was applied manually and falls between 4 and 5 milliseconds. This is in good agreement with Eq. 3.5 since there are miscellaneous influences that could to be accounted for (i.e., structures, temperature, reflections, etc.). 

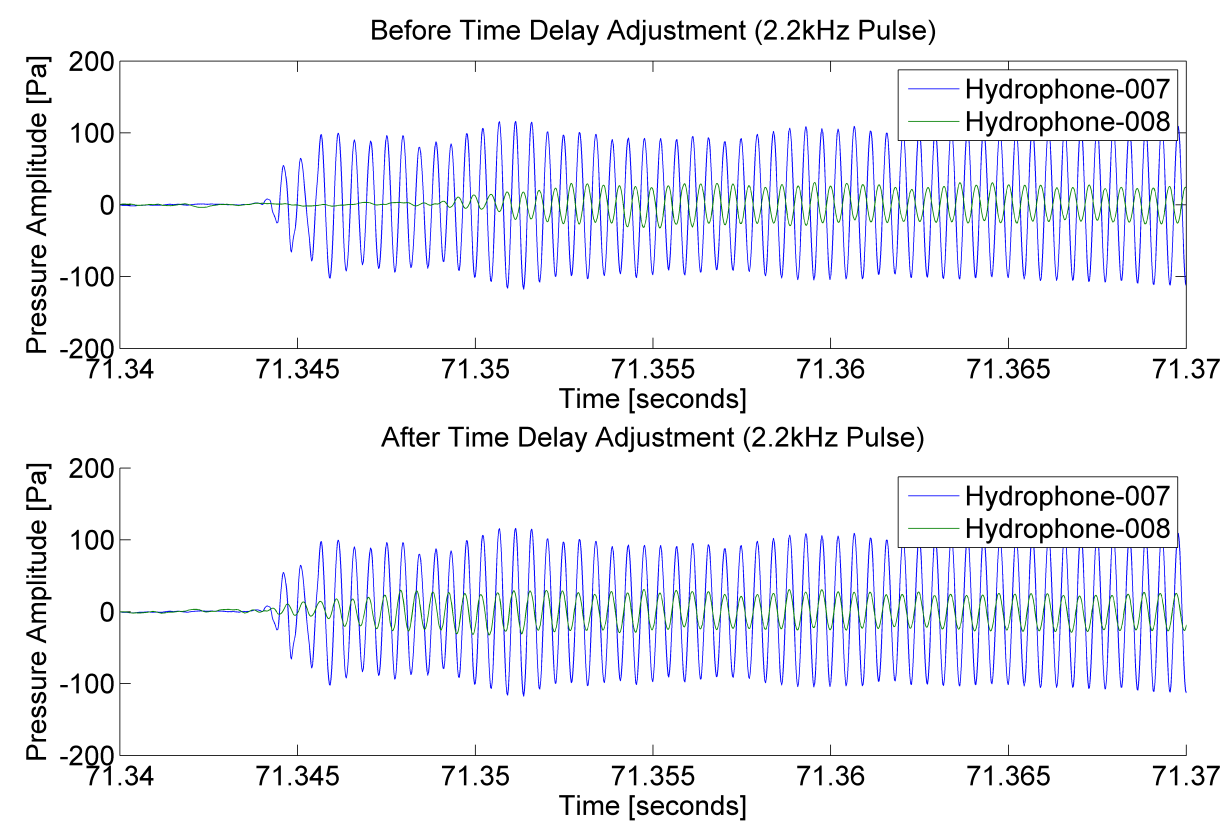

Figure 3.44: Time series showing the overlay of hydrophone-007 and -008 during the initiation of a reverberation measurement. The time delay between the hydrophones was approximated to be $4.8 \mathrm{~ms}$.
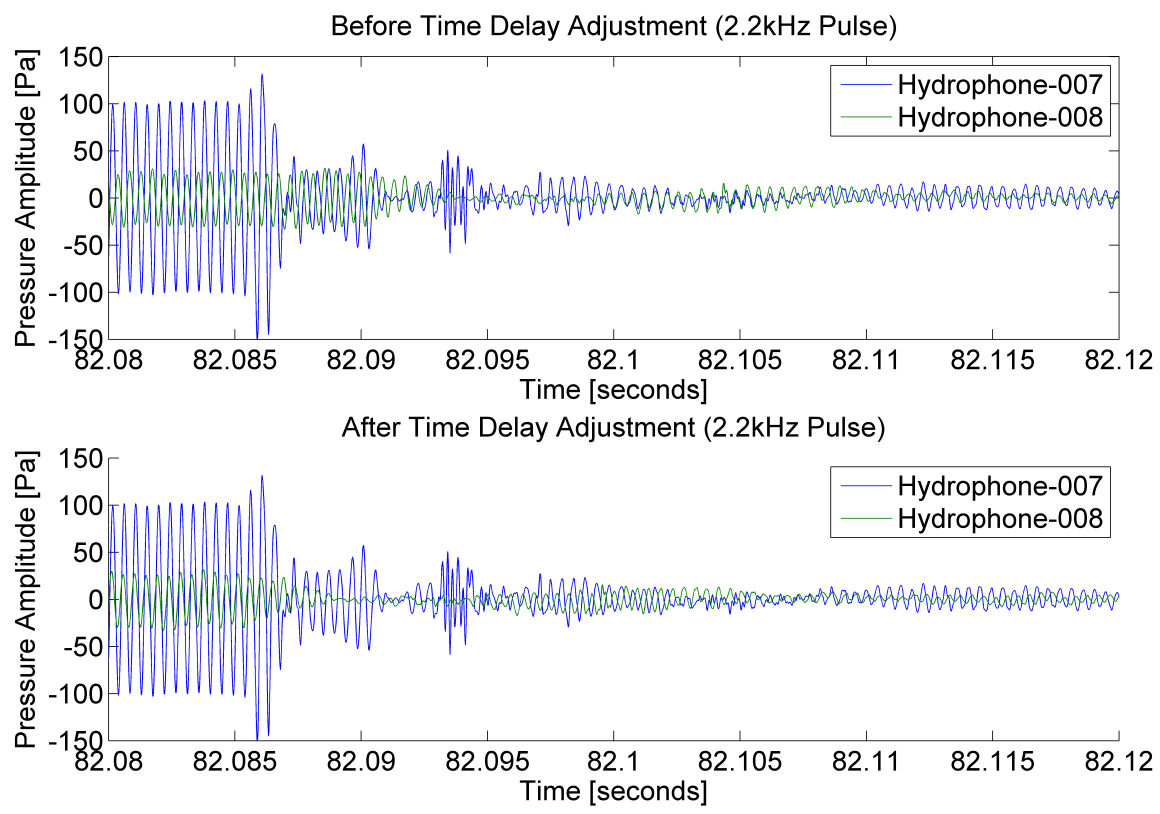

Figure 3.45: Time series showing the overlay of hydrophone-007 and -008 during the cutoff of a reverberation measurement. The time delay between the hydrophones was again approximated to be $4.8 \mathrm{~ms}$. Notice the complex nature of the waveform after cutoff. 


\section{Chapter 4 \\ Feasibility of Thermoacoustic Sensor Signal Detection}

The goal of the research at Penn State's Breazeale Nuclear Reactor is to determine if the frequency and amplitude of sound radiated from the thermoacoustic nuclear powered sensor (TAC Sensor) will be detectable above background noise levels created by pumps and machinery during normal operation. Previously in Chapter 3, background noise measurements were acquired using hydrophones placed at the bottom of the reactor's 70,000 gallon $\left(265 \mathrm{~m}^{2}\right)$ tank. Now, the largest question is whether the sound produced by a TAC sensor will be discernible among the predicted noise levels in the reactor pool. This chapter focuses on the power, pressure, and nuclear specifications cited previously in Chapter 2 , and utilizes the measurements of Chapter 3 to design a sensor to produce enough sound to telemeter frequency and amplitude levels to emulate temperature and neutron flux information.

\subsection{TAC Sensor Description}

The expected acoustical power output from such a TAC Sensor is calculated based on the design detailed in the Idaho National Laboratory Technical Report No. INL-LTD-15-34228 (March 2015) [21]. That sensor will contain two $7.2 \%$ enriched ${ }^{235} \mathrm{UO}_{2}$ pellets within a heat exchanger that is contained inside the thermoacoustic resonator. Each ${ }^{235} \mathrm{UO}_{2}$ pellet is approximately $5.0 \mathrm{~mm}$ in diameter and $10.0 \mathrm{~mm}$ long. The resonator will be pressurized to $2.0 \mathrm{MPa}$ (290 psia) with a mixture of $80 \%$ helium gas and $20 \%$ argon gas. The TAC sensor will be suspended resiliently within a cylinder that has the same dimensions as a Breazeale Reactor fuel-pin $(28.4 \mathrm{in}=72.1 \mathrm{~cm}$ long with an outside diameter of 1.446 in $=3.67 \mathrm{~cm}$ ) so it can be placed within the reactor's core among the other fuel pins. Figure 4.1 shows the section of the fuel pin that will contain the thermoacoustic resonator suspended at either end by two six-legged leaf springs. Figure 4.2 shows the details of the TAC Sensor's thermal core (i.e., stack and hot heat exchanger) and the surrounding thermal insulation space, without the $\mathrm{SiO}_{2}$ floss that suppresses buoyance-driven convection of the air trapped therein at atmospheric pressure. Figure 4.3 shows one of the suspension's leaf springs and 


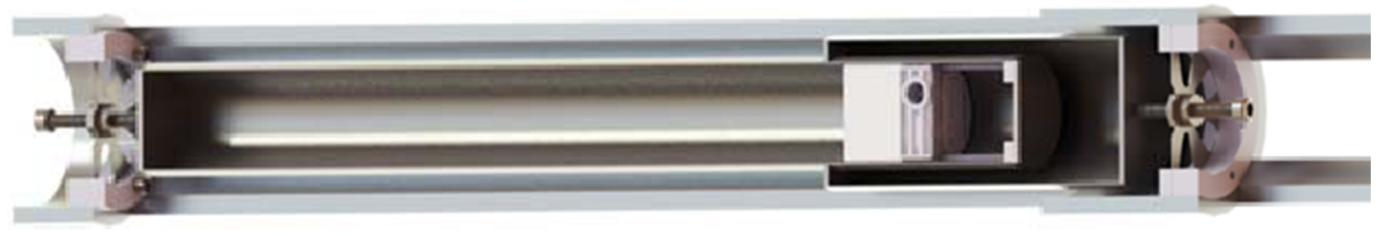

Figure 4.1: Full model of the thermoacoustic resonator to be tested in the Breazeale reactor pool. The ends of the resonator pictured show the leaf spring suspension system that connects the inside resonator to the slotted fuel pin. The fuel pin is slotted to permit sound propagation into the surrounding water. The stack and nuclear fuel enclosure are pictured on the right end of the resonator.

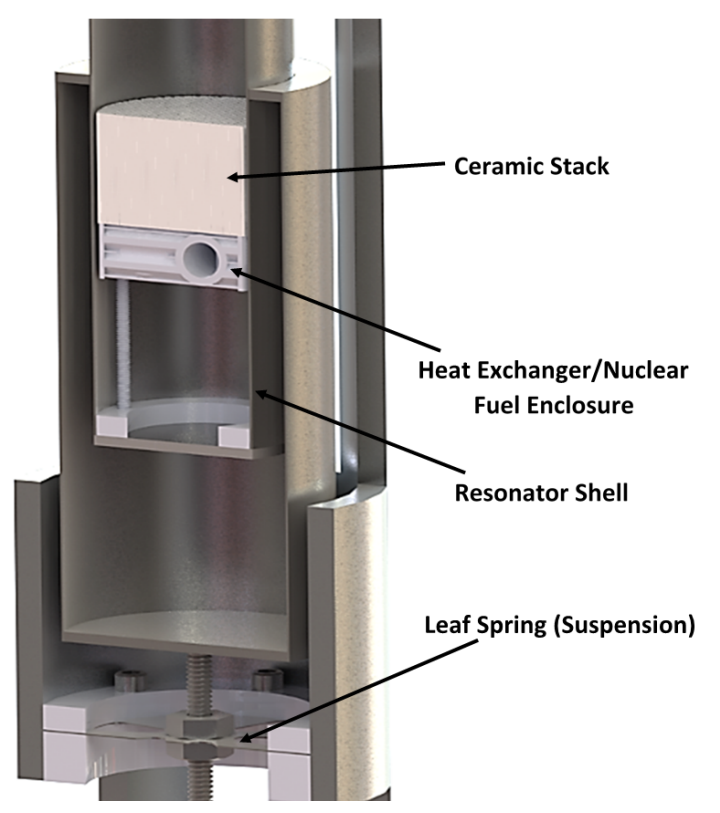

Figure 4.2: A zoomed-in view of the resonator shown in Fig. 4.1. The inner resonator is very similar to that of the Ali apparatus, apart from the heating element. The slotted outer shell is shown in contact with the leaf spring suspension system. This allows the resonator to oscillate without interference from the slotted outer shell.

the details of one-half of the heat exchanger that contains the $\mathrm{UO}_{2}$ pellets. 


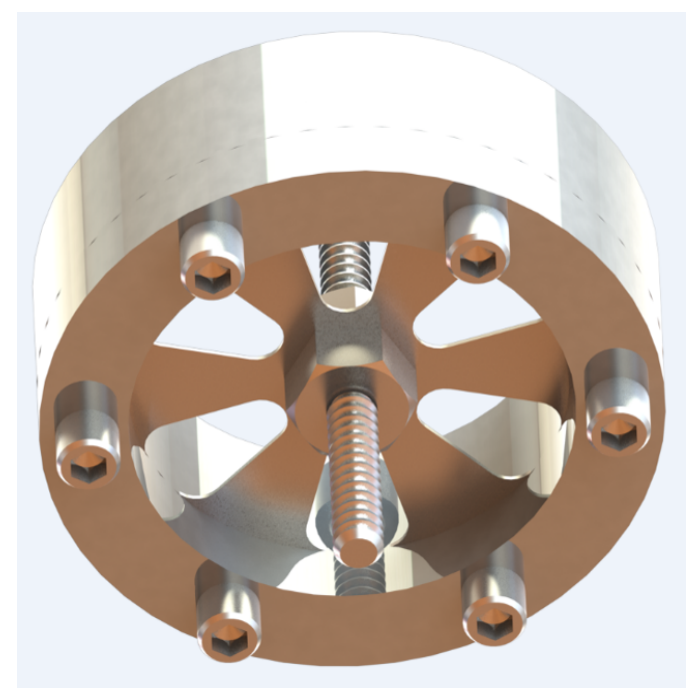

(a) Leaf spring

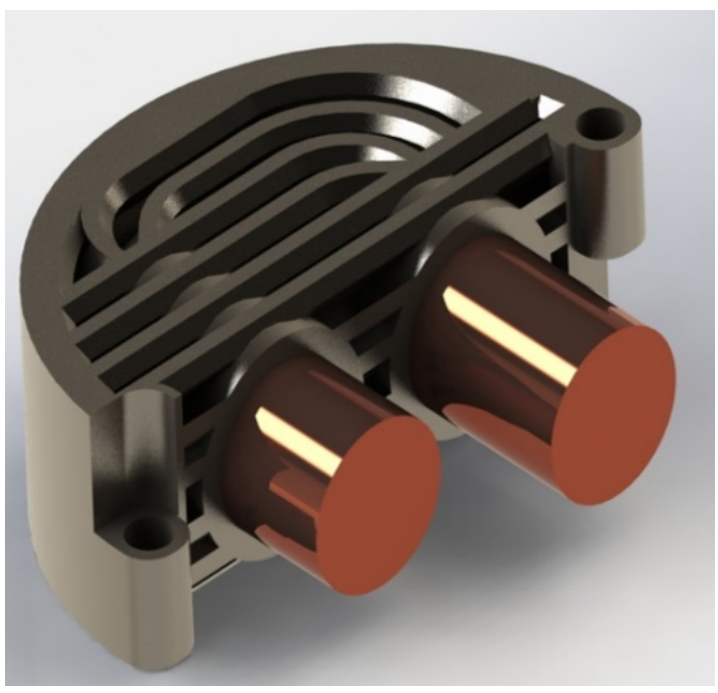

(b) Fuel enclosure

Figure 4.3: Leaf spring and fuel enclosure drawings for the fuel rod resonator. The leaf spring acts as the suspension system that allows the resonator to freely oscillate inside the slotted fuel pin. The fuel enclosure acts as both a casing for the nuclear pellets and a heatexchanger on the flowing gas in the resonator. The leaf spring is the connecting component between the resonator and the fuel pin.

\subsection{TAC Sensor Output Power and Detectability}

Heat is supplied to the thermoacoustic engine by the fission of the ${ }^{235} \mathrm{U}$ as described by the following typical nuclear reaction, initiated by the capture of a thermal neutron:

$$
n+{ }_{92}^{235} \mathrm{U} \rightarrow{ }_{56}^{141} \mathrm{Ba}+{ }_{36}^{92} \mathrm{Kr}+3 n+202.5 \mathrm{MeV}
$$

This reaction produces $19.54 \times 10^{12} \mathrm{~J} /$ mole of ${ }^{235} \mathrm{U}$.

Thermoacoustic oscillations of the gas are driven by the heat produced by the nuclear fission and will maintain an acoustic standing wave within the thermoacoustic resonator. That standing wave corresponds to about one half-wavelength of sound within the resonator, as shown in Fig. 4.4. The oscillatory momentum of the gas exerts a force on the resonator which will execute simple harmonic motion in the direction along the cylindrical resonator's axis. The motion is determined by the moving mass of the resonator and the entrained water outside the resonator. The stiffness of the suspension is sufficiently small that the amplitude of the resonator's oscillatory motion is controlled by the momentum of the gas and the mass of the resonator.

Another way to think about the motion of the resonator, caused by the standing acoustic wave, is to notice that the amplitude of the pressure at the ends of the resonator, indicated by the black line in Fig. 4.4, has opposite signs at opposite ends. Therefore, at one phase of the cycle, the pressure on the top end of the resonator is positive and on 


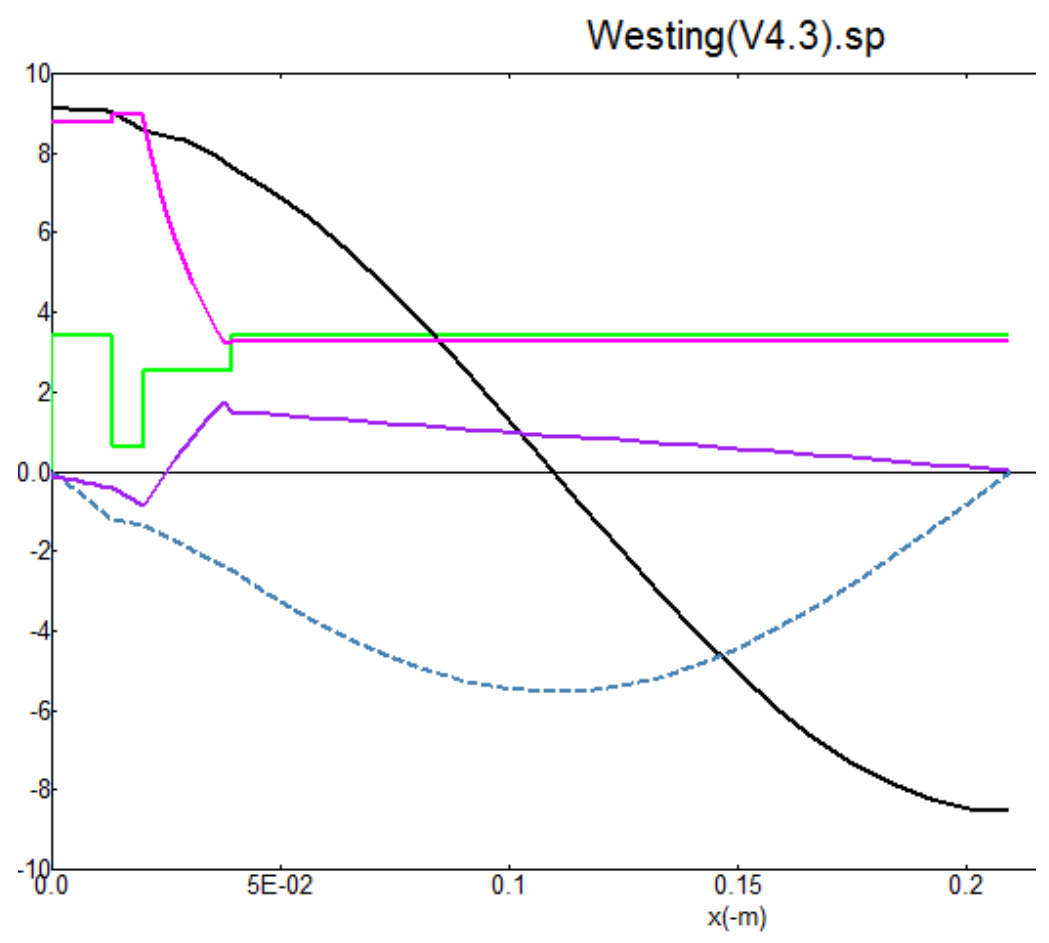

Figure 4.4: Acoustic standing wave predicted by DELTAEC within the nuclear-powered thermoacoustic sound source with an overall resonator length of $21 \mathrm{~cm}$ (10 in). The green line is the gas-filled cross-sectional area $\left(\times 10^{4} \mathrm{~m}^{2}\right)$, pink is absolute temperature $\left(\times 10^{-2} \mathrm{~K}\right)$, black is pressure amplitude $\left(\times 10^{-4} \mathrm{~Pa}\right)$, purple is acoustic power (watts), and dashed blue is acoustic volume velocity $\left(\times 10^{3} \mathrm{~m}^{3} / \mathrm{s}\right)$, all as a function of position within the resonator. The model predicts that the gas exerts a peak force of $57 \mathrm{~N}$ on the resonator at a frequency of $1,566 \mathrm{~Hz}$.

the bottom end is negative. At that time, the top of the resonator is "pushed" up and the bottom of the resonator is "sucked" up. A half-cycle later, the forces on the ends of the resonator are reversed. This shakes the resonator at the frequency of the standing wave.

The behavior of the resonator was modeled using the Los Alamos National Laboratory's Design Environment of Low-Amplitude Thermoacoustic Energy Conversion (DELTAEC) [25]. One such model predicts a resonance frequency of $1,588 \mathrm{~Hz}(\omega=10,000 \mathrm{rad} / \mathrm{s})$ when 25 watts of heat is delivered to the stack by the hot heat exchanger. The peak magnitude of the oscillatory force that the gas exerts on the resonator is about $F_{1}=57 \mathrm{~N}$. If the moving mass of the resonator (including the entrained waters hydrodynamic mass) is $m_{\text {res }}=0.25 \mathrm{~kg}$, then the acceleration of the resonator $a_{1}=F_{1} / m_{\text {res }}=228 \mathrm{~m} / \mathrm{s}^{2}$, corresponding to an oscillatory displacement amplitude $x_{1}=a_{1} / \omega^{2}=2.3 \times 10^{-6} \mathrm{~m}=2.3$ microns.

The resonator's volume is constant, so the resonator's translational oscillations generate a dipolar radiation field with the two ends of the resonator acting as two simple 
sources, oscillating $180^{\circ}$ out-of-phase, separated by the length of the resonator which is about $\mathrm{d}=25 \mathrm{~cm} \cong 10 \mathrm{in}$. At $1,588 \mathrm{~Hz}$, the wavelength of the sound in the water is $\lambda=\mathrm{c} / \mathrm{f} \cong 1,492 \mathrm{~m} / \mathrm{s} \div 1,588 \mathrm{~Hz} \cong 94 \mathrm{~cm}$. With the "sources" (i.e., the ends of the resonator) separated by about a quarter-wavelength of the sound in water, the dipole radiation approximation should be fairly accurate, despite the fact that the resonator is in contact with the surrounding water through slots in the fuel pin.

The radiated dipole power, $\Pi$, assuming an infinite medium, can be calculated by integration of the radiated pressure over all angles [14]:

$$
\Pi=\frac{\rho \omega^{4}\left(d U_{1}\right)^{2}}{12 \pi c^{3}}=\frac{\rho \omega^{2} V_{r e s}^{2}}{12 \pi c^{3}}\left(\frac{F_{1}}{m_{\text {res }}}\right)^{2}
$$

The right-hand version of Eq. 4.2 expresses the total radiated power in terms of the resonator's volume, $V_{\text {res }}$, and the density, $\rho$, and sound speed, $c$, of the water [21]. Using $F_{1}=57 \mathrm{~N}$ and $m_{\text {res }}=0.25 \mathrm{~kg}$, the total radiated acoustic power for a stack heat input power of $25 \mathrm{~W}$ is $\Pi=150 \mu \mathrm{W}$.

The root-mean-square steady-state diffuse pressure within the Breazeale Reactor's pool, $p_{r m s}(t=\infty)$ can be related to the total radiated power using Eq. 2.43 that is re-written below in terms of the exponentially determined energy relaxation time, $\tau=T_{60} / 13.82 \cong$ $8 \mathrm{~ms}$ and the volume, $V$, of the Breazeale Reactors pool. Again, $\rho$ is the density of water.

$$
p_{r m s}(t=\infty)=c \sqrt{\frac{\tau \rho_{0}}{V} \bar{\Pi}}
$$

Under these conditions (i.e., $25 \mathrm{~W}$ of stack heating), this corresponds to a diffuse steadystate acoustic pressure of $3.3 \mathrm{~Pa}$. Based on the background noise measurements reported in Chapter 3, this signal should be detectable within the reactor pool by a hydrophone placed on the bottom of the pool. After comparison of the average noise levels measured in the Breazeale Reactor Pool, which rarely exceeded $0.1 \mathrm{~Pa}$, and the calculations done by S. Garrett in the technical report for Idaho National Laboratory (INL/LTD-15-34228 [21]), the signal-to-noise ratio is predicted to be at least a factor of ten. And under the assumption that the ${ }^{235} \mathrm{U}$ pellets are heated at full reactor power $(1.0 \mathrm{MW})$, in the E-6 core location shown in Fig. 4.5, $120 \mathrm{~W}$ of thermal heating is expected for the pellets. Consequently, more than half of that maximum power will be deposited to the stack that should result in detectable acoustic resonance. 


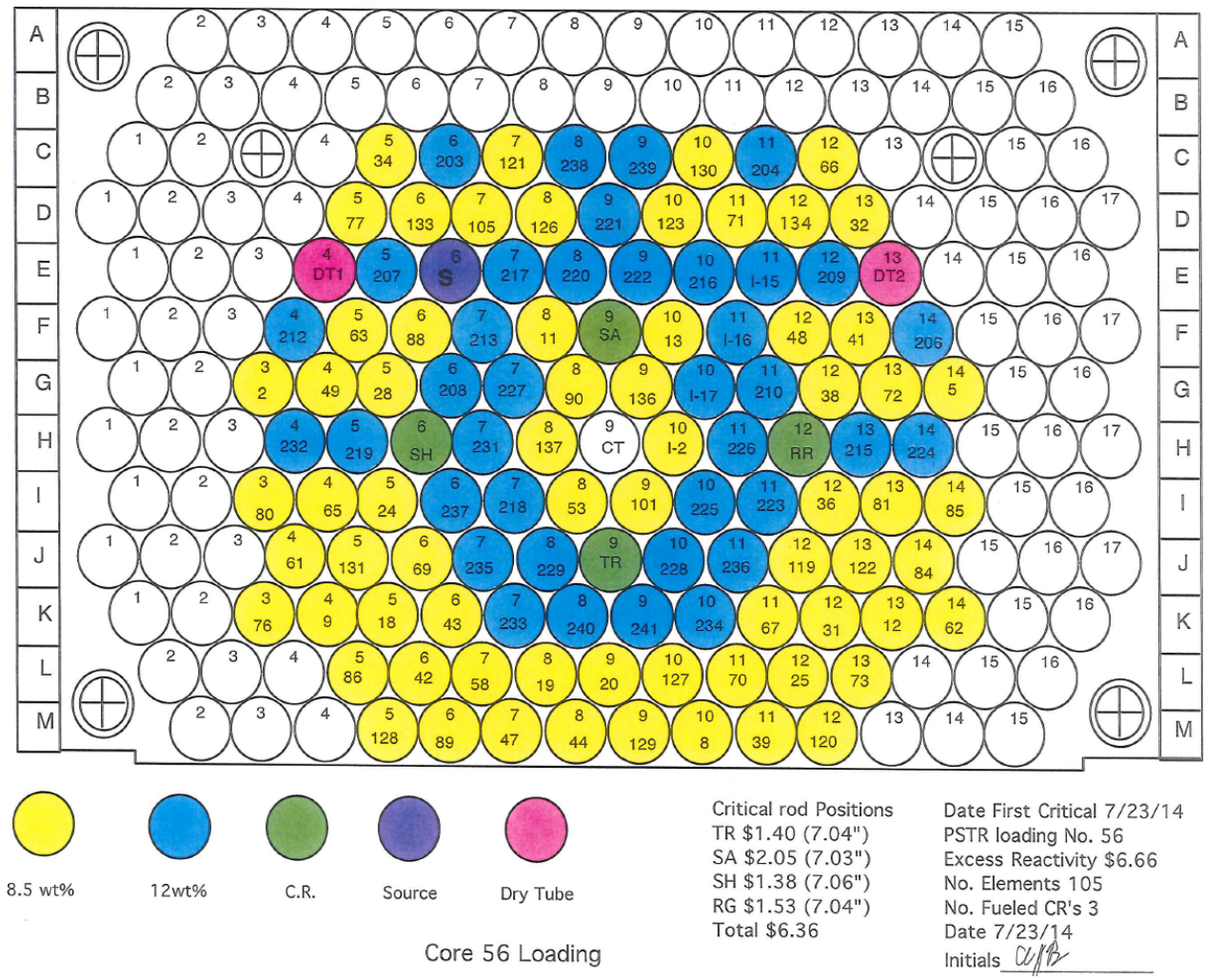

Figure 4.5: Fuel pin locations in the core of the Breazeale Nuclear Reactor. The TAC Sensor will be placed in the E-6 location. 


\section{Chapter 5 \\ Concluding Remarks}

\section{$5.1 \quad$ Summary}

The material presented in this thesis serves as a bridge between the measurements of the resonator fabricated by Ali and Garrett and a resonator that to be fabricated and inserted into the Breazeale reactor core. Ali also laid the groundwork for the thermoacoustic sensor: showing that it could track temperature fluctuations of the reactor's coolant using a resonator's frequency modulated resonance frequency (see Fig. 5.1). Ali's work ended with the modeling, fabrication, and initial testing of a thermoacoustic resonator in a controlled environment; however, he was not able to test the device in the Breazeale reactor $[2]$.

Ali showed that thermoacoustic resonance can function while being electrically heated and immersed in a calorimeter full of water. The theory and experimentation conducted here have taken his experiment a step further by placing a sound source in the Breazeale reactor's pool and testing acoustic behavior with active background noise sources. The theoretical and experimental results were then unified to create a singular prediction for the behavior of a nuclear powered, thermoacoustic, fuel rod resonator. The nuclear and thermoacoustic estimates made in Chapter 4 predict the onset of acoustic oscillations for a TAC Sensor to be placed in the Breazeale reactor pool. The detection of an acoustic signal produced by such a device in the reactor pool will prove the long-coming hypothesis made by S. Garrett in March 2011 - that a thermoacoustic resonator can be powered by nuclear fuel and detected in the harsh environment that is a nuclear reactor.

The Fukushima disaster triggered the original research on nuclear powered thermoacoustic resonators, but the convenience and availability of the Breazeale reactor was the impetus that sparked Ali's work toward full-scale implementation. In a pressurized water reactor the study of acoustic behavior would be difficult due to the sealed enclosure; whereas the open-pooled design of the Breazeale pool makes the theoretical and experimental processes manageable. This pressure-release boundary was one of many important distinctions in the Breazeale reactor, as well as its accessibility and low-danger environment.

First, a rectangular geometry was assumed before the prediction of modal behavior 


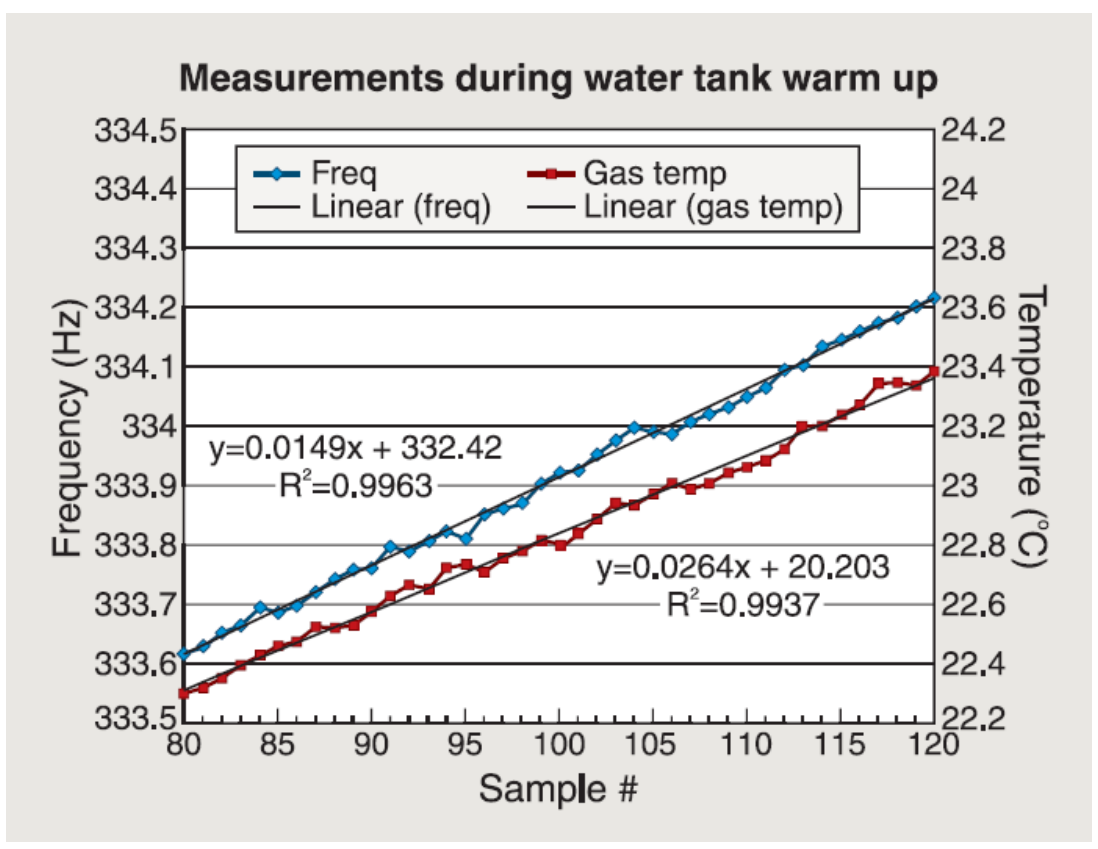

Figure 5.1: Plot made by R. Ali for a thermoacoustic resonator in a calorimeter. Temperature and frequency are shown in red and blue, respectively. The resonator was electrically heated and showed linearity in both frequency and gas temperature. The ability to track these changes suggested an auspicious future and motivated this current thesis.

inf the pool, which was important for identifying an appropriate frequency range for the fuel rod resonator. The modal density of the pool indicated that the acoustic resonator's frequency would not be disturbed by large structural resonances, an indication that the resonance frequency of the resonator would fall in the statistical domain of the pool modes (diffuse sound field). Consequently, an investigation of statistical energy mechanisms in the pool established distinct parameters such as: $\tau, T_{60}$, and $A_{a b s}$. The absorption in the enclosure was studied so that average energy approximations could be made for the system. This would return the efficiency of the thermoacoustic resonator's output power and indicate the detectability of the sensor.

A steady-state analysis of the pool was used to determine the critical distance, $r_{d}$, which is used as the region where the statistical regime dominates over direct sound propagation between source and receiver. It was proposed that when a receiver is placed in the diffuse field, the reactor pool would have an equal influence on frequencies being emitted. Following the critical distance, the Schroeder frequency was found and the acoustic characterization of the reactor pool was complete. The focus then shifted to basic resonator design and the considerations involved in making the TAC device.

The dipole assumption is an approximation for the resonator's radiation characteristics. It was found that the total radiated sound power from the resonator is proportional to the heat input and pressure of the gas inside the resonator. The radiated sound power is also inversely proportional to the molecular mass of the gas and the mass of the resonator 
$[21]$.

The optimization of the resonator power shaped the fabrication of the fuel rod resonator. For example, a higher pressurization of the internal gas is likely, a gas with a small atomic mass will likely be used, and the resonator will be designed such that its mass is as small as possible. These design parameters and optimizations helped bring the theoretical discussion to a close. The attention then shifted toward the experiments to be conducted in the Breazeale reactor pool. The experiments served as the link between the theory and future experimentation with the resonator in the reactor pool.

On November 12, 2014, background noise measurements proved that hydrophones submerged in the Breazeale reactor pool were capable of measuring calibrated acoustic signals. It was also concluded that the noise produced by various pumps is generally broadband with some low frequency tonals. This low frequency content will likely be filtered out using an analog filter so that the low frequency amplitudes don't cause clipping during measurement. The accelerometers and hydrophones showed very good signal-to-noise ratio during the different measurements.

The first series of measurements involved the emission of three separate tones that were not harmonically related. The first measurements were designed to view the average noise level of the different pumps. The greatest amount of noise (with the most pumps running) never exceeds $0.5 \mathrm{~Pa}$ (and rarely exceeds $0.1 \mathrm{~Pa}$ ) at the hydrophones. The detectability of an acoustic signal in the Breazeale reactor pool is therefore dependent upon the signal's ability to overcome this background noise. The tri-tone configuration suggested that a dipolar acoustic sound source is capable of being detected with maximal background noise in the reactor pool.

Following the tri-tone measurements, a reverberation analysis was conducted by using the steady-state cutoff method. This involved the emission of a particular frequency for an extended period of time. The goal was to set-up the steady-state field of the enclosure. Then, the signal is abruptly terminated, and the subsequent decay is recorded. This decay information characterized the energy absorption in the reactor pool and helped find an average energy expression for the resonator.

Chapter 3 concludes with a discussion of various data analysis techniques and signal processing options. Section 3.3.1 focuses on the challenges involved in obtaining reverberation times for the original hydrophone configuration. The direct sound decay was observed and then resolved by the relocation of hydrophone-008 to a location in the pool distant from local noise sources. Filtering techniques and a peak-finder algorithm results are shown, followed by the discussion of real-time analysis methods. The spectrogram and power spectrum are both essential tools for monitoring acoustic signals, and in the absence of either, the complete characterization of a potential time varying frequency signal is difficult. A short analysis of time delay is also included as the last section in Chapter 3. 
Finally, Chapter 4 connects both Chapter 2 and Chapter 3 through exploration of the thermoacoustic nuclear fuel rod resonator. Scale drawings of the proposed resonator are shown, while a short synopsis of the nuclear and acoustic calculations were included as well. Chapter 4 is important because it brings R. Ali's resonator one step closer toward implementation inside a nuclear reactor, while also incorporating the theory and experiments produced here in this thesis. The predictions and expected performance of the fuel rod resonator are addressed in the Idaho National Laboratory Technical Report [21]. The majority of the work presented here functions as a bridge connecting the work conducted by Ali and Garrett, to the future fabrications and testing of the thermoacoustic resonator in the Breazeale reactor core. Correspondingly, the nuclear powered acoustic resonator is currently being fabricated for insertion into the Breazeale reactor core at Penn State. This thermoacoustic device was proved operational in September of 2015.

\subsection{Conclusion and Future Work}

The thermoacoustic fuel rod resonator began as a response to the devastating Fukushima disaster, but quickly developed into a potential revolution in the nuclear industry. This thesis serves as a bridge between the experiments conducted by R. Ali and S. Garrett and the future testing of a thermoacoustic resonator inside the Breazeale reactor core. Ali and Garrett worked with an electrically heated resonator that they submerged into a calorimeter filled with water. They were able to show that the resonance frequency of the resonator changed as the surrounding coolant temperature changed. These results encouraged the exploration of the Breazeale reactor and its acoustic properties. And consequently, the acoustic characterization of the Breazeale reactor pool was initiated.

The predictions in the INL technical report [21] were calculated using the experiments and derivations in this thesis. These predictions also guided the design of the resonator shown in Fig. 4.1, which was fabricated and then tested in the Breazeale reactor in September 2015. This resonator will operated above the background noise levels in the reactor pool, and will be further studied using advanced signal processing in the future. Each nuclear fuel pellet is predicted to supply approximately $55 \mathrm{~W}$ of heating power, and consequently, high optimism resulted in the onset of acoustic oscillations.

Upon acoustic onset, the results from Chapter 2 and 3 become highly relevant in the analysis of the acoustic signals. The diffuse field distance (i.e., the critical distance, $r_{d}$ ) will be used as the minimum distance a sensor must be placed from the resonator. The predicted dipolar behavior will also be taken into account during measurement, which could affect the recorded amplitude depending on the measurement angle. Additionally, the resonance frequency and amplitude of the device will need to be tracked with high accuracy.

In the recent past, a thermoacoustic fuel rod resonator was fabricated and tested in the Breazeale nuclear reactor at Penn State. The resonator resembled the drawings in Figs. 4.1 and 4.2. This resonator was placed in the E-6 position of the core (see Fig. 4.5). 
Once acoustic oscillations began, a data acquisition system, designed by Idaho National Laboratory, recorded the signal in real time. The power spectrum, spectrogram, and time series will be utilized to monitor the signals.

Once the sensors produce signals, it is imperative that the signal processing utilizes proper tracking of frequency as well as amplitude. It is likely that Idaho National Lab and the Pennsylvania State University will work together to produce a proper software program to track and monitor the signals, and therefore, both temperature and neutron fluxes in a reactor.

In closing, the acoustic environment studied in this thesis suggested that TAC sensor signals would be detectable. As a result of this, a full-scale nuclear fuel rod resonator was fabricated and verified experimentally. The signal processing techniques implemented in this thesis proved to be essential tools for tracking and monitoring different processes in a nuclear reactor, based solely on the frequency and amplitude behavior of the thermoacoustic resonator. Nonetheless, acoustic resonance in the Breazeale reactor pool is not only feasible, but possible. And in the future the thermoacoustic nuclear powered resonator will continue to be explored and will likely be evaluated using of the techniques developed in this thesis. 
Appendices 


\section{Appendix A Sensor Calibration}

Table A.1 lists the transducers that were used during the experiments. Sensitivity measurements were conducted before and after the experimentation, and all calibrations remained stable within experimental uncertainty. There were no equipment failures. The calibration sensitivities were averaged and used to convert the measured voltages to pascals (for the hydrophones) and acceleration to Gs (for the accelerometer).

The accelerometer was calibrated using a B\&K-4294 exciter. The exciter provided 10 $\mathrm{m} / \mathrm{s}^{2}( \pm 2 \%)$ at $1,000 \mathrm{rad} / \mathrm{s}=159.2 \mathrm{~Hz}$. The hydrophone calibration required the use of a reference microphone, an ACO Pacific $1 / 2$ in., with a sensitivity that was validated using a B\&K-4228 Pistonphone. The Pistonphone created $31.0 \mathrm{~Pa}_{r m s}$ at $250 \mathrm{~Hz}$. The hydrophones were inserted into a coupler along with the ACO 1/2 in. microphone and attached to another B\&K Pistonphone (type-4223). The Pistonphone created $134.9 \mathrm{~Pa}$ at $250 \mathrm{~Hz}$.

\begin{tabular}{|c|c|c|c|c|c|}
\hline Instrumentation & Model\# & Serial \# & $\begin{array}{c}\text { Sensitivity } \\
\text { Prior }\end{array}$ & $\begin{array}{c}\text { Sensitivity } \\
\text { After }\end{array}$ & $\begin{array}{c}\text { Sensitivity } \\
\text { Factory }\end{array}$ \\
\hline $\begin{array}{c}\text { Underwater } \\
\text { Sound Source }\end{array}$ & Lubell & LL9162T & N/A & N/A & N/A \\
\hline Microphone & ACO Pacific & ACOJ-7013 & $12.56 \mathrm{mV} / \mathrm{Pa}$ & $12.49 \mathrm{mV} / \mathrm{Pa}$ & $12.6 \mathrm{mV} / \mathrm{Pa}$ \\
\hline Hydrophone-007 & HTI-96_MIN & 613007 & $2.94 \mathrm{mV} / \mathrm{Pa}$ & $2.89 \mathrm{mV} / \mathrm{Pa}$ & $2.85 \mathrm{mV} / \mathrm{Pa}$ \\
\hline Hydrophone-008 & HTI-96_MIN & 613008 & $2.74 \mathrm{mV} / \mathrm{Pa}$ & $2.74 \mathrm{mV} / \mathrm{Pa}$ & $2.72 \mathrm{mV} / \mathrm{Pa}$ \\
\hline Pistonphone & B\&K-4228 & 1918508 & N/A & N/A & N/A \\
\hline Pistonphone & B\&K-4223 & 1379453 & $\mathrm{~N} / \mathrm{A}$ & $\mathrm{N} / \mathrm{A}$ & $\mathrm{N} / \mathrm{A}$ \\
\hline Exciter (Accel) & B\&K-4294 & 1759853 & $\mathrm{~N} / \mathrm{A}$ & $\mathrm{N} / \mathrm{A}$ & $\mathrm{N} / \mathrm{A}$ \\
\hline Accelerometer & PCB-321A02 & 4326 & $95.1 \mathrm{mV} / \mathrm{g}$ & $96.7 \mathrm{mV} / \mathrm{g}$ & $96 \mathrm{mV} / \mathrm{g}$ \\
\hline
\end{tabular}

Table A.1: Calibration and instrumentation for the November 12, 2014 and December 4, 2014 experiments. The top four transducers were used in the experiments, and the bottom three were used as calibration devices before and after the experiments. 


\section{Appendix B \\ Instrumentation used for Reactor Vibroacoustic Measurements}

To ensure repeatability of the experiments in the reactor pool, a block diagram of the instrumentation has been included, as well as a 3-D AutoCAD ${ }^{\circledR}$ drawing of the reactor pool. The outputs of the transducers were monitored in real time on a Kikisui cos6100A oscilloscope, and an Agilent 35670A 4-channel dynamic signal analyzer. All signals were recorded at CD quality $(44.1 \mathrm{ks} / \mathrm{s}, 16$-bit) and were recorded simultaneously by a 4 -channel Roland R-44 digital recorder. Fig. B.1 outlines the data flow and setup during the experiments in the reactor pool. After the experiments on November 12th and December 4th,

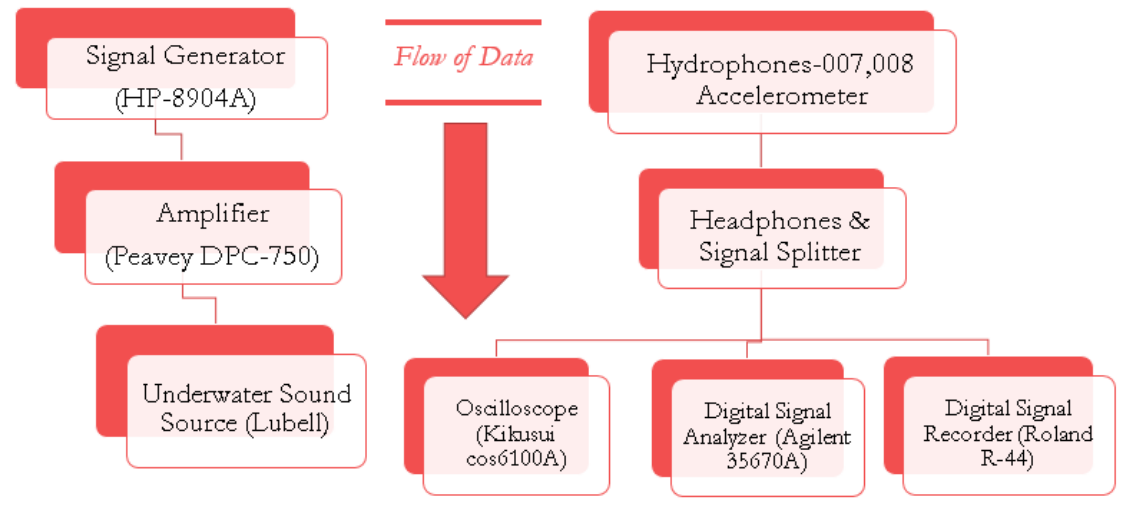

Figure B.1: Data flow during the Breazeale Reactor Pool measurements. The oscilloscope, digital signal analyzer, and digital recorder were hardware that were cross referenced for real-time monitoring and post-processing analysis.

the data from the Digital Signal Recorder was taken and compared with that of the HP Digital Signal Analyzer (DSA). After concluding that the data from the Roland and DSA agreed, MATLAB ${ }^{\circledR}$ was used to produce the plots seen in this thesis. Figures B.3-B.6 map out the experiments conducted, and also give approximate locations for the noise sources such as pump outlets, suction inlets, and a discharge header. The reactor drawings also show the reactor core (where the fuel rod resonator will eventually be placed). 


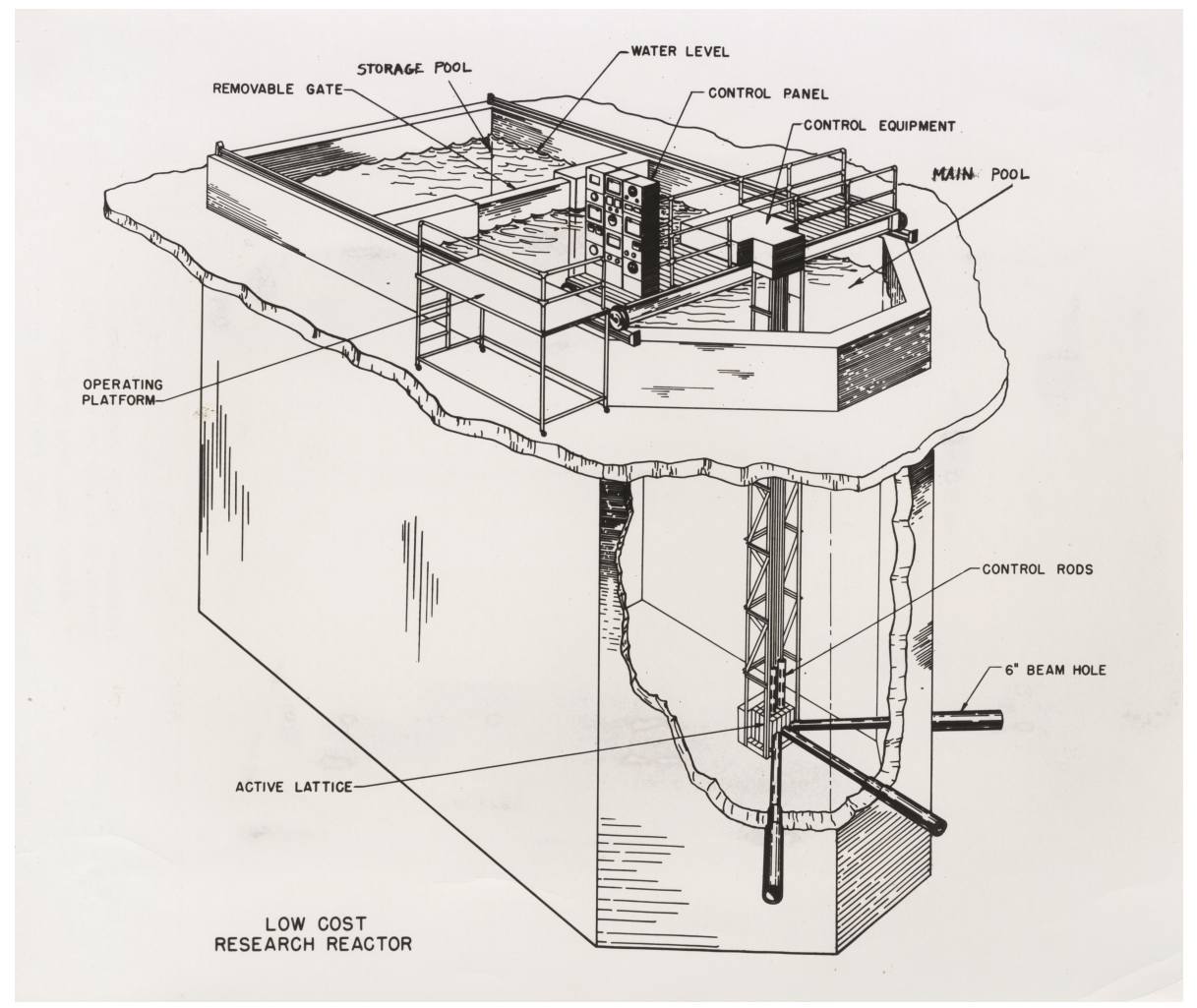

Figure B.2: Classic drawing of the Breazeale Reactor [24]. The figure shows all of the essential functions of an open-pooled research reactor. It was also a model for the drawings made in Figs. B.3-B.6.

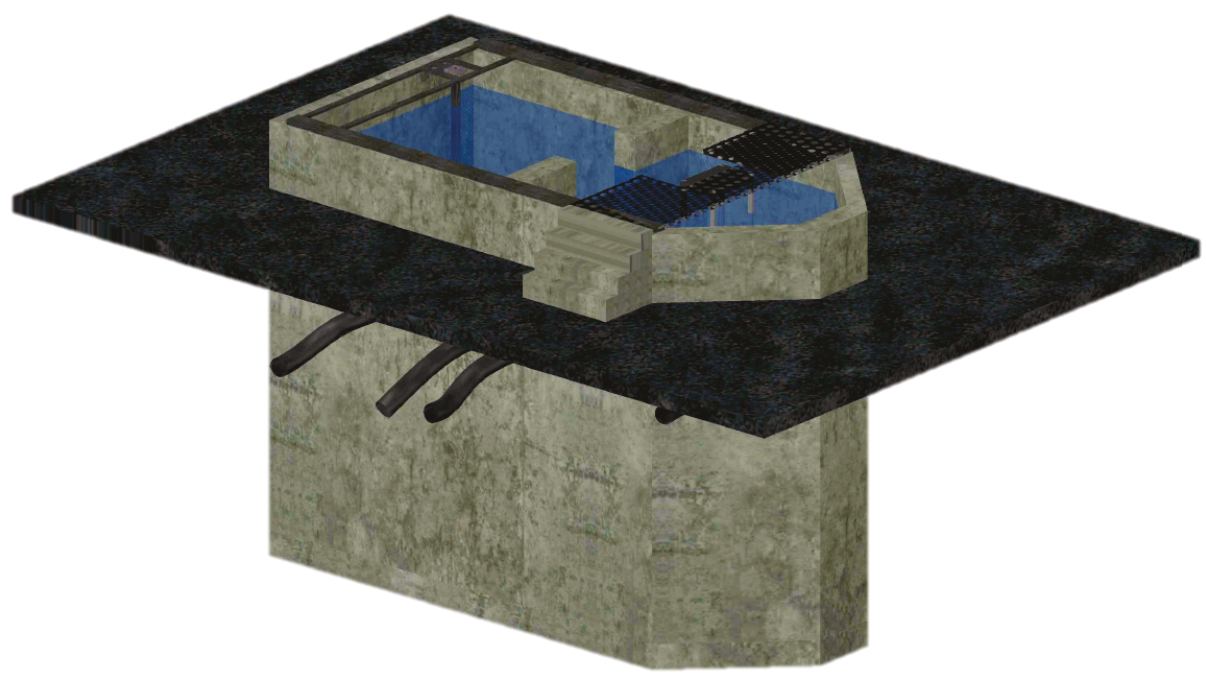

Figure B.3: AutoCAD ${ }^{\circledR}$ drawing of the Breazeale Reactor Pool with the floor, walls, and main infrastructure. 


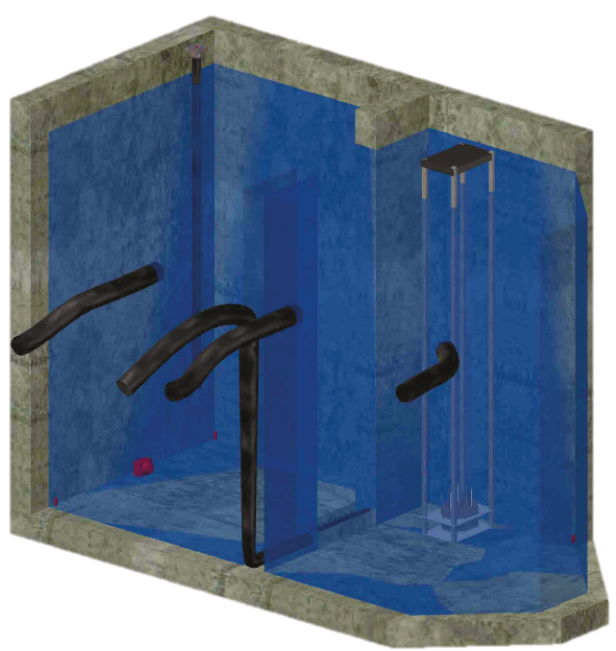

Figure B.4: AutoCAD ${ }^{\circledR}$ drawing of the Breazeale Reactor Pool with the sides taken down. This view allows one to view the pumps, transducers, and the core.

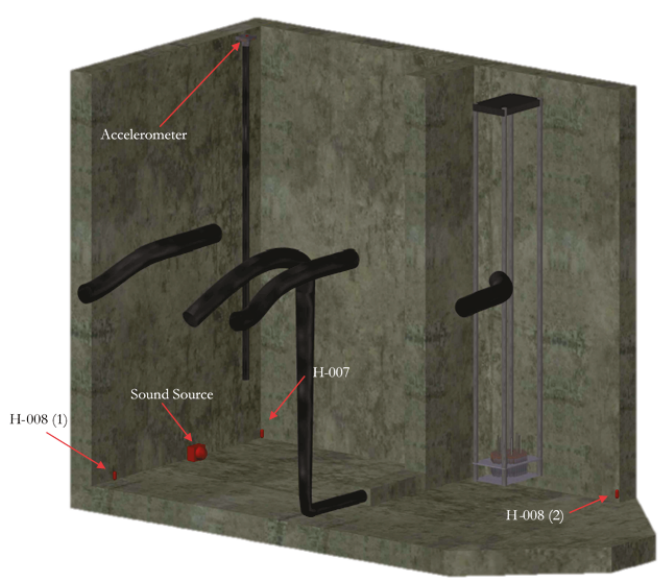

Figure B.5: Labeled transducers with the reactor core and pumps in view. This is identical to Fig. 3.2 in Sec. 3.2.1. 


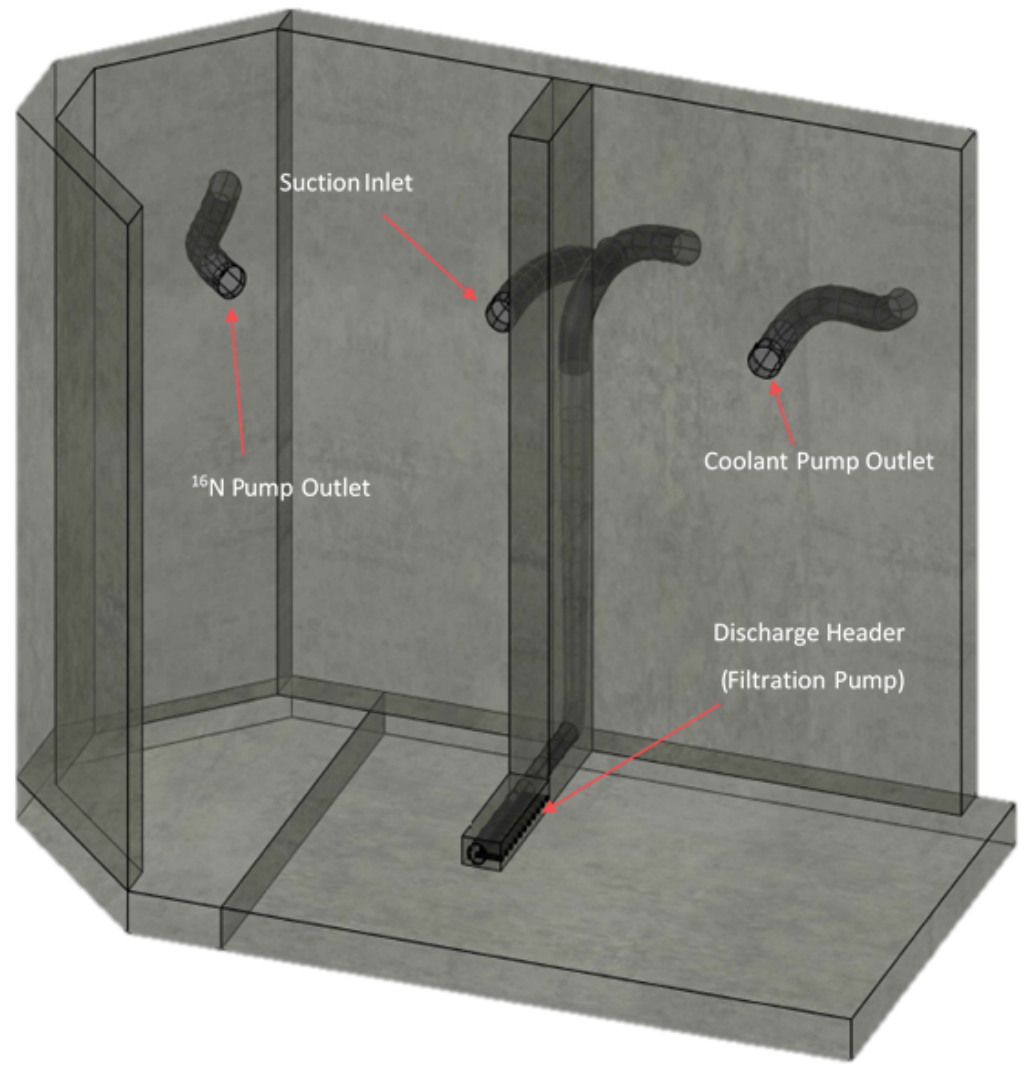

Figure B.6: Labeled pumps and their location in the reactor pool. The N-16 diffuser pump was the most significant noise source of the three pumps (see Sec. 3.2). 


\section{Bibliography}

[1] Mirion Technologies (IST Canada), Sensing Systems, 465 Dobbie Drive, Cambridge, Ontario N1R 5X9, Canada. https://www.mirion.com.

[2] ALI, R. Self-Powered Thermoacoustic Sensor for In-Pile Nuclear Reactor Monitoring. Master's thesis, Pennsylvania State University, 2013.

[3] Ali, R. A., Garrett, S. L., Smith, J. A., And Kotter, D. K. Thermoacoustic Thermometry for Nuclear Reactor Monitoring. IEEE Instrumentation $\&$ Measurement Magazine 16, 3 (2013).

[4] Blackstock, D. T. Fundamentals of Physical Acoustics. John Wiley \& Sons, Inc., 2000.

[5] Energy Information Administration. How much electricity does a nuclear power plant generate?, 2015 (accessed November 12, 2015).

[6] Garrett, S. Thermoacoustic Nuclear Power Measurement Assembly (TNPMA). Statement-Of-Work, Pennsylvania State University, 2013.

[7] Greenspan, M. Simple Derivation of the Boltzmann-Ehrenfest Adiabatic Principle. Journal of the Acoustical Society of America 27, 34 (1955).

[8] Heibel, M. D., Flammang, R. W., and Sumego, D. M. Thermo-Acoustic Nuclear Power Distribution Measurement Assembly, October 2014. Patent. US 2014/0321591 A1.

[9] Heibel, M. D., Flammang, R. W., and Sumego, D. M. Thermo-Acoustic Nuclear Power Distribution Measurement Assembly, December 2014. Patent. US 2014/0362965 A1.

[10] IAEA. The International Nuclear and Radiological Event Scale, 2015 (accessed May $5,2015)$.

[11] Acoustics Measurement of room acoustic parameters Part 1: Performance spaces. Standard, International Organization for Standardization, 2009. 
[12] Kurokawa, K. The Official Report of the Fukushima Nuclear Accident Independent Investigation Commission. Tech. rep., The National Diet of Japan.

[13] Morse, P. M. Vibration and sound. American Institute of Physics, 1981, ch. 8.

[14] Morse, P. M., And Ingard, K. U. Theoretical acoustics. McGraw-Hill, 1968, ch. 9.

[15] Pierce, A. D. Acoustics. Acoustical Society of America, 1994, ch. 6.

[16] Ratnam, R., Jones, D., Wheeler, B., O’Brien, W., Lansing, C., And Feng, A. Blind estimation of reverberation time. Journal of The Acoustical Society of America 114,5 (2003).

[17] Rayleigh, J. W. S. B. The Theory of Sound, Volume II. Second ed. Macmillan, 1896, p. 226.

[18] Rossing, T. D. Springer handbook of acoustics. First ed. Springer Science + Business Media, LLC, 2007, pp. 244-248.

[19] Sabine, W. C. Collected papers on acoustics. Cambridge: Harvard University Press, 1922, ch. 1.

[20] Schroeder, M. R. The "Schroeder Frequency" revisited. Journal of The Acoustical Society of America 99, 5 (1996).

[21] Smith, J., Heidrich, B., Garrett, S., Smith, R., Poese, M., and Hrisko, J. Report on the TAC Sensor Design for the Breazeale Reactor Demonstration, INLLTD-15-34228. Tech. rep., Idaho National Laboratory, March 2015.

[22] Smith, J., Kotter, D., Ali, R., And Garrett, S. Synergistic Smart Fuel For In-pile Nuclear Reactor Measurements. Oct 2013.

[23] Swift, G. Thermoacoustic engines. Journal of The Acoustical Society of America 84 (1988).

[24] Wank, A. Atoms for Peace at Penn State, 2010 (accessed May 5, 2015).

[25] Ward, W., Clark, J., and Swift, G. Design Environment for Low-amplitude Thermoacoustic Energy Conversion (DeltaEC), Ver. 6.3b11.9. Los Alamos National Lab Report LA-CC-01-13 (2010). 